Hélio Jacinto da Cruz Neto

\title{
Otimização do Posicionamento de Sensores e Atuadores Para o Controle com Realimentação de Saída Utilizando Critério de Desempenho Quadrático
}



Hélio Jacinto da Cruz Neto

\section{Otimização do Posicionamento de Sensores e Atuadores Para o Controle com Realimentação de Saída Utilizando Critério de Desempenho Quadrático}

Dissertação apresentada à Escola de Engenharia de São Carlos da Universidade de São Paulo como parte dos requisitos para a obtenção do título de Mestre em Engenharia Mecânica.

Área de concentração: Dinâmica das Máquinas e Sistemas.

Orientador: Marcelo Areias Trindade 
AUTORIZO A REPRODUÇÃO TOTAL OU PARCIAL DESTE TRABALHO, POR QUALQUER MEIO CONVENCIONAL OU ELETRONNICO, PARA FINS DE ESTUDO E PESQUISA, DESDE QUE CITADA A FONTE.

Ficha catalográfica elaborada pela Biblioteca Prof. Dr. Sérgio Rodrigues Fontes da EESC/USP com os dados inseridos pelo(a) autor(a).

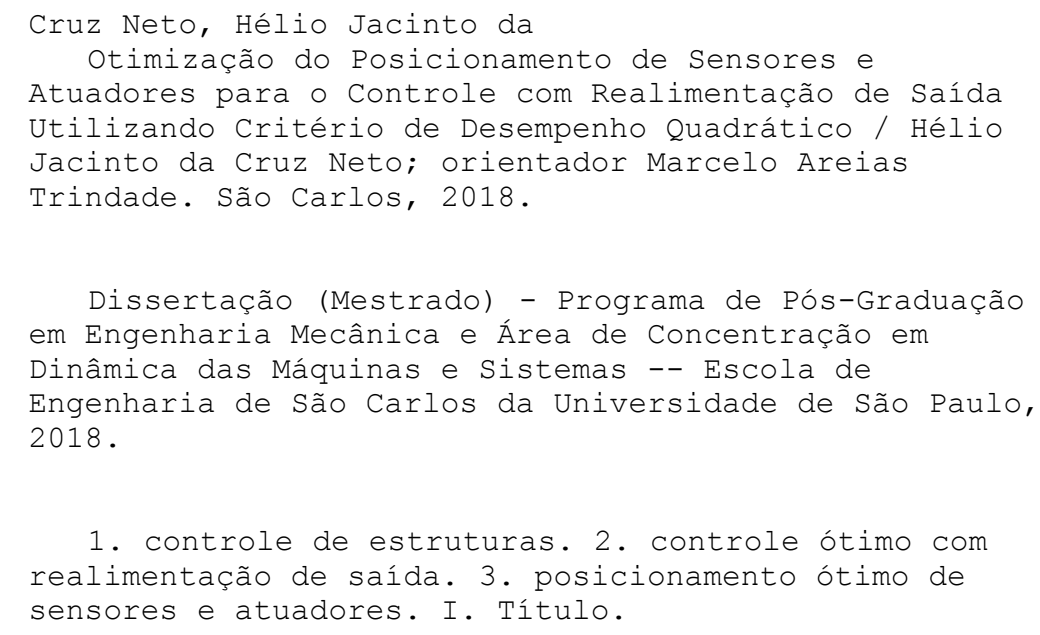

1. controle de estruturas. 2. controle ótimo com realimentação de saída. 3. posicionamento ótimo de sensores e atuadores. I. Título.

Eduardo Graziosi Silva - CRB - 8/8907 


\section{FOLHA DE JULGAMENTO}

Candidato: Engenheiro HELIO JACINTO CRUZ NETO.

Título da dissertação: "Otimização do posicionamento de sensores e atuadores para o controle com realimentação de saída utilizando critério de desempenho quadrático".

Data da defesa: 02/03/2018.

\section{Comissão Julgadora:}

Prof. Associado Marcelo Areias Trindade (Orientador)

(Escola de Engenharia de São Carios/EESC)

Prof. Dr. Alberło Luiz Serpa

(Universidade Estadual de Campinas/UNICAMP)

Profa. Dra. Tatiana de Figueiredo Pereira Alves Taveira Pazelli (Universidade Federal de São Carlos/UFSCar)
Resultado:

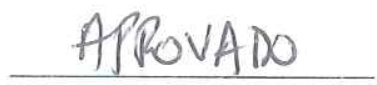

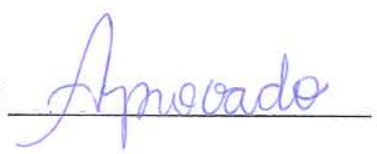

Coordenador do Programa de Pós-Graduação em Engenharia Mecânica: Prof. Associado Gherhardt Ribałski

Presidente da Comissão de Pós-Graduação: Prof. Associado Luis Fernando Cosła Alberto 

Para minha avó, Augusta Bianquine, pessoa mais sábia que conheci. 



\section{Agradecimentos}

Agradeço aos meus pais pelo carinho e cuidado. Sou muito grato de ter pais atenciosos e prestativos que se dedicaram para que eu pudesse ter oportunidades que eles não tiveram. Vocês são um exemplo para mim. Agradeço também a minha irmã Isabel por ser a melhor irmã que eu poderia ter.

Agradeço ao Prof. Marcelo Trindade pelo comprometimento como orientador e pesquisador. Ele me possibilitou as oportunidades que precisava para desenvolver a pesquisa da melhor maneira possível, além de ter paciência, flexibilidade, confiança e disponibilidade para diversas reuniões. Agradeço também pelos conselhos profissionais e pela dedicação além da pesquisa.

Agradeço a minha companheira Nathália pelo carinho e suporte. Você foi a pessoa que esteve mais próxima nas diversas circunstâncias relacionadas ao trabalho e fora dele, e sabe de vários pormenores do seu desenvolvimento. Obrigado por me acompanhar e cuidar de mim em todos esses momentos.

Agradeço aos demais professores e funcionários do Departamento de Engenharia Mecânica pela contribuição na minha formação.

Agradeço aos colegas de laboratório e amigos pelos conselhos e compreensão.

Agradeço aos membros da banca pela disponibilidade para ler e avaliar o meu trabalho.

Agradeço ao Conselho Nacional de Desenvolvimento Científico e Tecnológico (CNPq) pelo apoio financeiro. 

"Os outros meninos, um queria ser médico, outro pirata outro engenheiro, ou advogado, ou general. Eu queria ser um pajem medieval... Mas isso não é nada. Pois hoje eu queria ser uma coisa mais louca: eu queria ser eu mesmo" 



\section{Lista de ilustrações}

Figura 1 - Deslocamento e deflexão definidos ao longo de um elemento $j$ da viga. . 47

Figura 2 - Coordenadas locais e globais para um elemento retangular em uma placa. 48

Figura 3 - Viga engastada com sensores e atuadores colocalizados. . . . . . . . . 56

Figura 4 - Viga engastada com atuador e sensores pontuais. . . . . . . . . . 65

Figura 5 - Forma dos modos de vibrar de uma viga engastada-livre. . . . . . . . . 69

Figura 6 - Comparação entre as respostas temporais considerando a pior condição inicial do controle LQRv. . . . . . . . . . . . . . . . . . . . . . . . . 71

Figura 7 - Comparação entre os esforços de controle para a pior condição inicial do controle LQRv. . . . . . . . . . . . . . . . . . . . . 71

Figura 8 - Razão entre critérios de desempenho do controle colocalizado $\left(J_{c}\right)$ e do LQR $\left(J_{l}\right)$ em função do ganho de realimentação de velocidade. . . . . . 72

Figura 9 - Comparação entre as respostas temporais considerando a pior condição inicial da proposta Levine-Athans em relação ao LQR. . . . . . . . . . 73

Figura 10 - Comparação entre os esforços de controle considerando a pior condição inicial da proposta Levine-Athans em relação ao LQR. . . . . . . . . . 73

Figura 11 - Comparação entre as respostas temporais para uma condição inicial dada aproximadamente por uma velocidade inicial no quinto modo. . . 74

Figura 12 - Comparação entre os esforços de controle para uma condição inicial dada aproximadamente por uma velocidade inicial no quinto modo. . . 75

Figura 13 - Comparação entre as propostas de Levine-Athans e minmax através do diagrama de Bode. . . . . . . . . . . . . . . 76

Figura 14 - Comparação entre as respostas temporais para uma condição inicial dada aproximadamente por uma velocidade inicial no primeiro modo. . 77

Figura 15 - Comparação entre os esforços de controle para uma condição inicial dada aproximadamente por uma velocidade inicial no primeiro modo. . 78

Figura 16 - Resposta temporal para o controle com realimentação de saída com dois sensores e o LQR, considerando uma entrada impulsiva aplicada na extremidade livre da viga. . . . . . . . . . . . . . . . . . . 78

Figura 17 - Esforço de controle para o LQR e o controle com realimentação de saída com dois sensores, considerando uma entrada impulsiva aplicada na extremidade livre da viga. . . . . . . . . . . . . . .

Figura 18 - Comparação entre o LQR e o controle com realimentação de saída com dois sensores através do diagrama de Bode.

Figura 19 - Máximo valor da razão entre as funções custo do controle de saída e LQR quando os ganhos dos sensores são variados. . . . . . . . . . . . . 81 
Figura 20 - Máximo valor da razão entre as funções custo do controle de saída e LQR quando as posições dos sensores são variadas. . . . . . . . . . . . 82

Figura 21 - Comparação entre as respostas temporais dos sistemas com parâmetros ótimos e com erro de $3 \%$ para uma entrada impulsiva na extremidade livre. . . . . . . . . . . . . . . . . . . . . 82

Figura 22 - Comparação entre os esforços de controle dos sistemas com parâmetros ótimos e com erro de $3 \%$ para uma entrada impulsiva na extremidade livre. . . . . . . . . . . . . . . . . . . . 83

Figura 23 - Comparação das respostas temporais entre LQR e controle com realimentação de saída para uma condição inicial dada aproximadamente por uma velocidade inicial no $14^{\circ}$ modo. . . . . . . . . . . . . . . . . . 85

Figura 24 - Comparação dos esforços de controle entre LQR e controle com realimentação de saída para uma condição inicial dada aproximadamente por uma velocidade inicial no $14^{\circ}$ modo. . . . . . . . . . . . . . . . 85

Figura 25 - Comparação das respostas temporais entre LQR e controle com realimentação de saída para uma condição inicial dada aproximadamente por uma velocidade inicial no $1^{\circ}$ modo. . . . . . . . . . . . . . . . 86

Figura 26 - Comparação dos esforços de controle entre LQR e controle com realimentação de saída para uma condição inicial dada aproximadamente por uma velocidade inicial no $1^{\circ}$ modo. . . . . . . . . . . . . . . 86

Figura 27 - Sistema de coordenadas e dimensões de uma placa retangular simplesmente apoiada em todos os lados. . . . . . . . . . . . . . . . . . . 87

Figura 28 - Modos de vibrar para uma placa simplesmente apoiada. . . . . . . . . 90

Figura 29 - Comparação entre as respostas temporais para a condição inicial 1 considerando medida de deslocamento na posição $\boldsymbol{\alpha}_{r}$. . . . . . . . . . . 91

Figura 30 - Comparação entre os esforços de controle para a condição inicial 1. . 92

Figura 31 - Comparação entre as respostas temporais para a condição inicial 7 considerando medida de deslocamento na posição $\boldsymbol{\alpha}_{L A}$. . . . . . . . . . 92

Figura 32 - Comparação entre os esforços de controle para a condição inicial $7 . \quad$. . 93

Figura 33 - Comparação entre as respostas temporais de um ponto na posição $\boldsymbol{\alpha}_{r}$ considerando a pior condição inicial do controle com realimentação de saída. . . . . . . . . . . . . . . . . . . . . . . 94

Figura 34 - Comparação entre os esforços de controle considerando a pior condição inicial do controle com realimentação de saída. . . . . . . . . . . . . . . 94

Figura 35 - Comparação entre as respostas em frequência considerando a entrada e a saída na posição $\boldsymbol{\alpha}_{r}$. . . . . . . . . . . . . . . . . . . . . . . 95

Figura 36 - Máximo valor da razão entre as funções custo do controle de saída e LQR quando os ganhos dos sensores são variados. . . . . . . . . . . . . 96 
Figura 37 - Máximo valor da razão entre as funções custo do controle de saída e LQR quando a posição do sensor 1 é variada. . . . . . . . . . . . . 96

Figura 38 - Máximo valor da razão entre as funções custo do controle de saída e LQR quando a posição do sensor 2 é variada. . . . . . . . . . . . . . . 97

Figura 39 - Comparação entre as respostas temporais dos sistemas com parâmetros ótimos e com erro de $5 \%$. . . . . . . . . . . . . . . . . . 98

Figura 40 - Comparação entre os esforços de controle dos sistemas com parâmetros ótimos e com erro de $5 \%$. . . . . . . . . . . . . . . . . . . . 98

Figura 41 - Comparação das respostas temporais entre LQR e controle com realimentação de saída para uma condição inicial dada aproximadamente por uma velocidade inicial no $2^{\circ}$ modo. . . . . . . . . . . . . . . . . . . 99

Figura 42 - Comparação dos esforços de controle entre LQR e controle com realimentação de saída para uma condição inicial dada aproximadamente por uma velocidade inicial no $2^{\circ}$ modo. . . . . . . . . . . . . . . . 100

Figura 43 - Comparação das respostas temporais entre LQR e controle com realimentação de saída para uma condição inicial dada aproximadamente por uma velocidade inicial no $40^{\circ}$ modo. . . . . . . . . . . . . . . 100

Figura 44 - Comparação dos esforços de controle entre LQR e controle com realimentação de saída para uma condição inicial dada aproximadamente por uma velocidade inicial no $40^{\circ}$ modo. . . . . . . . . . . . . . . . . . 101

Figura 45 - Comparação entre LQR e controle com realimentação de saída em $2 k H z$ através do diagrama de Bode. . . . . . . . . . . . . . . . 101 



\section{Lista de tabelas}

Tabela 1 - Características da viga estudada. . . . . . . . . . . . . 67

Tabela 2 - Comparação entre os valores de frequência teóricos e os obtidos pelo modelo em elementos finitos. . . . . . . . . . . . . . . 67

Tabela 3 - Comparação entre as funções custo para as propostas Levine-Athans e $\operatorname{minmax} \ldots \ldots \ldots \ldots . \ldots \ldots 75$

Tabela 4 - Comparação entre as funções custo do controle colocalizado e controle com dois sensores utilizando a proposta minmax. . . . . . . . . . . . 77

Tabela 5 - Principais resultados para a viga engastada-livre. . . . . . . . . . . 80

Tabela 6 - Comparação de desempenho entre o controle colocalizado e o LQR variando o número de modos. . . . . . . . . . . . . . . . . 83

Tabela 7 - Comparação de desempenho entre o controle com dois sensores e o LQR variando o número de modos. . . . . . . . . . . . . . . . . . . 84

Tabela 8 - Características da placa estudada. . . . . . . . . . 88

Tabela 9 - Comparação entre os valores de frequência teóricos e os obtidos pelo modelo em elementos finitos. . . . . . . . . . . . . . . . . 89

Tabela 10 - Comparação entre as funções custo para as propostas Levine-Athans e robusta. . . . . . . . . . . . . . . . . . 9 91

Tabela 11 - Parâmetros ótimos e comparação com o LQR para a placa simplesmente apoiada variando o número de sensores. . . . . . . . . . . . 93 



\section{Lista de abreviaturas e siglas}

LQR Linear Quadratic Regulator

LQG Linear Quadratic Gaussian

LIT Linear e Invariante no Tempo

DFP Davidon-Fletcher-Powell

BFGS Broyden-Fletcher-Goldfarb-Shanno

BMI Bilinear Matrix Inequalities

HIFOO H-Infinity Fixed-Order Optimization

PPF Positive Position Feedback

IHR Improving Hit and Run

SQP Sequential Quadratic Programming

FEM Finite Element Method

HJB Hamilton-Jacobi-Bellman

clf control Lyapunov function

ARE Algebraic Riccati Equation 



\section{Resumo}

Cruz Neto, H.J. Otimização do Posicionamento de Sensores e Atuadores para o Controle com Realimentação de Saída Utilizando Critério de Desempenho Quadrático. Dissertação (Mestrado) - Escola de Engenharia de São Carlos, Universidade de São Paulo, São Carlos, 2018.

Estruturas flexíveis estão sujeitas a excitações desconhecidas que podem causar danos. Um dos possíveis artifícios para lidar com este problema é a teoria de controle de sistemas dinâmicos. Em particular, uma técnica que suscita o interessa para aplicação nesta classe de sistemas é o controle ótimo, devido às suas boas propriedades de resposta e factibilidade, podendo ser aplicado até através de circuitos analógicos. O contratempo desta técnica é a necessidade de um número de sensores igual ao número de estados do sistema, o que para estruturas é inviável. Como uma alternativa, pode se empregar os procedimentos usuais de restrição de realimentação do sinal medido. No entanto, estes casos não consideram o projeto das matrizes de saída e entrada, fator determinante para o controle de vibrações em estruturas. O objetivo deste trabalho é preencher esta lacuna. Inicialmente, são introduzidos alguns conceitos das teorias de controle ótimo, dinâmica estrutural e sobre métodos de discretização em séries. Em seguida, determinam-se as condições necessárias de otimalidade considerando como variáveis de otimização o ganho e as posições dos sensores e atuadores. Determinadas as condições, investigam-se os principais desafios para solução destas equações, dados pela existência de parâmetros que estabilizem o sistema e a dependência do ponto ótimo em relação à condição inicial do sistema. O primeiro é resolvido a partir da especificação do sistema linear para uma forma modal e utilizando funções de controle de Lyapunov, o que adicionalmente proporciona o resultado de que o controle colocalizado é um controle ótimo. Para o segundo são propostas duas soluções, sendo uma utilizada para determinar as posições dos atuadores para projetar um controle LQR com desempenho satisfatório, e a outra para determinar os ganhos e posições dos sensores de modo a obter um controle com realimentação de saída com desempenho próximo ao LQR projetado. Os resultados obtidos a partir da aplicação da metodologia desenvolvida em exemplos da dinâmica estrutural revelaram um desempenho notável. Mesmo para uma razão pequena entre o número de sensores pelo número de estados obteve-se um desempenho equivalente ao LQR, exibindo também propriedades robustez consideráveis em relação às variáveis de otimização. Conclui-se que a metodologia desenvolvida é uma boa alternativa para as técnicas de controle LQR e LQG.

Palavras chave: controle de estruturas. controle ótimo com realimentação de saída. posicionamento ótimo de sensores e atuadores. 



\section{Abstract}

Cruz Neto, H.J. Optimal Placement of Sensors and Actuators for the Output Feedback Control Using Quadratic Performance Criterion. Master Thesis - São Carlos School of Engineering, University of São Paulo, São Carlos, 2018.

Flexible structures are subject to unknown excitations that may cause damage. One of the possible artifices to deal with this problem is the control theory of dynamical systems. In particular, a technique that raises the interest for application in this class of systems is the optimal control, due to its good properties of response and feasibility, as it can be applied even through analog circuits. A drawback of this technique is the need for a number of sensors equal to the number of states, which for structures is impracticable. As an alternative, the usual procedures of using only measured signals for feedback can be employed. However, these cases do not consider the design of the input and output matrices, a determining factor for vibration control in structures. The purpose of this paper is to fill this gap. Initially, some concepts of the theories of optimal control, structural dynamics and series discretization methods are introduced. Then, the optimality conditions are determined considering the gain and locations of sensors and actuators as the optimization variables. Given these conditions, we investigate the main challenges to solve these equations, given by the existence of parameters that stabilize the system and the dependence of the optimum point in relation to the initial condition of the system. The first one is solved from the specification of the linear system to a modal form and using Lyapunov control functions, which additionally provides the result that the collocated control is an optimal control. For the second two solutions are proposed, one being used to determine the positions of the actuators to design a LQR control with satisfactory performance, and the other to determine the gains and positions of the sensors in order to obtain an output feedback control with close performance to the designed LQR. The results obtained from the application of the methodology developed in structural dynamics examples revealed a remarkable performance. Even for a small ratio between the number of sensors by the number of states a performance equivalent to the LQR was obtained, also exhibiting considerable robustness properties in relation to the optimization variables. It is concluded that the developed methodology is a good alternative for LQR and LQG control techniques.

Keywords: structural control. optimal output feedback control. sensor and actuator placement optimization. 



\section{Sumário}

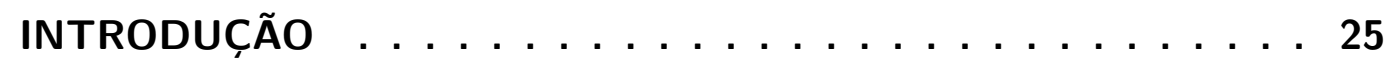

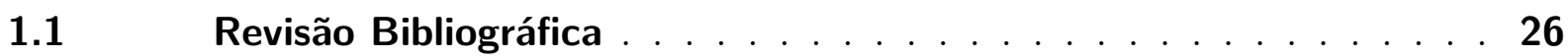

1.1.1 Controle Ótimo com Realimentação da Saída . . . . . . . . . . . . . . 27

1.1.2 Aplicações no Controle de Vibrações em Estruturas e Posicionamento de Sensores e Atuadores . . . . . . . . . . . . . . . . . . . . . . . 29

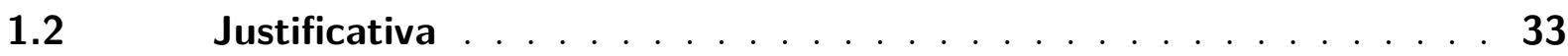

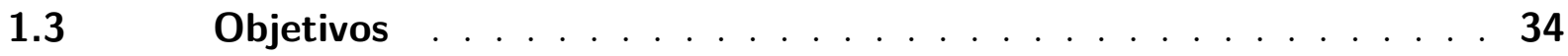

2 TÓPICOS DA TEORIA DE CONTROLE ÓTIMO E DINÂMICA

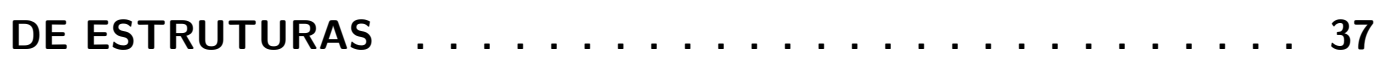

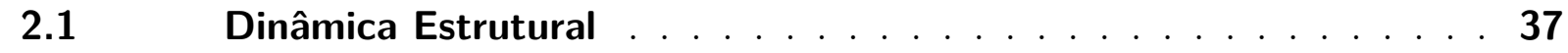

$2.2 \quad$ Métodos de discretização em séries . . . . . . . . . . . . . . 41

$2.3 \quad$ Regulador Linear Quadrático . . . . . . . . . . . . . . . . . 49

3 POSICIONAMENTO DE SENSORES E ATUADORES NO CONTROLE COM REALIMENTAÇÃO DE SAÍDA . . . . . . . . . 53

3.1 Condições Necessárias para Minimização da Função Custo . . . . 53

3.2 Estabilização de Estruturas Flexíveis . . . . . . . . . . . . 55

$3.3 \quad$ Condição Inicial do Sistema . . . . . . . . . . . . . . . . . 58

3.3.1 Aproximação entre os Controles com Realimentação de Saída e de Estados 59

3.3.2 Conjunto de Possíveis Condições Iniciais . . . . . . . . . . . . . . . 61

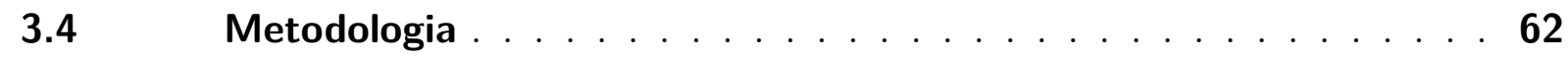

4 APLICAÇÕES NO CONTROLE DE VIBRAÇÃO DE ESTRUTU-

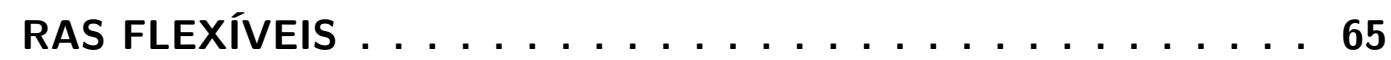

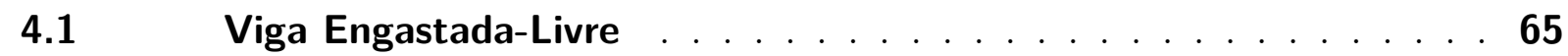

4.1.1 Modelo e Projeto do Sistema de Controle . . . . . . . . . . . . . . 65

$4.1 .2 \quad$ Variação de Parâmetros . . . . . . . . . . . . . . . . . 80

$4.2 \quad$ Placa Simplesmente Apoiada . . . . . . . . . . . . 86

4.2.1 Modelo e Projeto do Sistema de Controle . . . . . . . . . . . . . . 87

4.2.2 Variação de Parâmetros . . . . . . . . . . . . . . . . . . 95

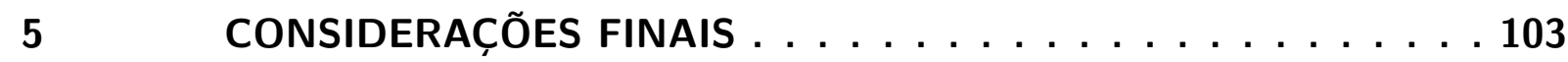

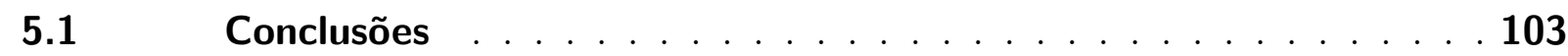

$5.2 \quad$ Principais Contribuições . . . . . . . . . . . . . . . . . 104

$5.3 \quad$ Trabalhos Futuros . . . . . . . . . . . . . . . . . . . 104 
REFERÊNCIAS ........................ 107 


\section{Introdução}

Vibrações são caracterizadas por movimentos oscilatórios que um sistema pode exibir em torno de um ponto de equilíbrio. Em alguns poucos casos este comportamento é desejado, como na vibração de um motor desbalanceado de um celular, de palhetas em um instrumento de sopro ou do diafragma de um alto-falante. No entanto, na maioria dos casos este fenômeno é indesejado, podendo causar perda de energia, fadiga, ruído e imprecisão de posicionamento. No campo de controle estrutural, cujo objeto de estudo é o controle de vibrações em estruturas flexíveis, desenvolveu-se a seguinte terminologia para classificar as técnicas utilizadas para mitigar ou suprimir a vibração:

- Controle Ativo: No controle ativo há a presença de sensores e atuadores. Utilizase uma fonte externa para amplificar o sinal dos sensores e fornecer potência ao atuador. O sinal enviado ao atuador para corrigir a configuração do sistema é uma função dos sinais medidos pelos sensores.

- Controle Passivo: O controle passivo é caracterizado pela ausência de sensores e atuadores. Acoplam-se elementos passivos à estrutura como elementos viscoelásticos ou inerciais a fim de alterar as frequências de ressonância e aumentar o amortecimento. Os principais dispositivos de controle passivo são tratamentos amortecedores e isoladores dinâmicos.

- Controle Semiativo: No controle semiativo são utilizados materiais cujos valores de rigidez e amortecimento podem ser alterados através de uma fonte externa de baixa potência, atuando como elementos passivos controláveis (HOUSNER et al., 1997). Esta abordagem impede que se forneça energia ao sistema, garantindo que este permaneça estável para uma entrada limitada. Alguns exemplos de dispositivos de controle semiativo são amortecedores com fluido magnetoreológico e pistões hidráulicos controláveis.

- Controle Híbrido: O controle híbrido combina as técnicas de controle ativo e passivo. Uma estrutura com elementos viscoelásticos distribuídos, além de pastilhas piezelétricas operando como sensores e atuadores pode ser considerada como um exemplo de controle híbrido.

O foco deste trabalho será o controle ativo de vibrações em estruturas descritas por um sistema linear de equações diferenciais; no entanto, os resultados aqui obtidos podem ser estendidos para o controle híbrido caso algumas propriedades do sistema de controle sejam mantidas. 
Um dos principais métodos de controle ativo é a retroalimentação, ou feedback, no qual os sinais medidos pelos sensores representam estados do sistema e estes são combinados e enviados ao atuador. A forma como estes sinais são combinados, ou a representação matemática que descreve a atuação em função dos sinais medidos é chamada de lei de controle. Existem diversas técnicas que permitem projetar diferentes leis de controle para um sistema, como backstepping, sliding mode, LQR, LQG, $\mathrm{H}_{\infty}$, entre outras. Este trabalho irá tratar do controle ótimo, que abrange todo o conjunto de técnicas que visa determinar uma lei de controle de forma a minimizar um critério de desempenho.

Diversos critérios já foram empregados e alguns deles acabaram se popularizando devido a sua factibilidade e obtenção de boas propriedades de resposta. Destes, pode se destacar o critério de desempenho quadrático, o qual é caracterizado por um funcional quadrático nos estados do sistema e nas variáveis de controle. Esta formulação visa uma relação de compromisso entre a velocidade em que o sistema em malha fechada se aproxima da sua posição de equilíbrio e o esforço empregado. Apesar de ser largamente utilizado em vários problemas de controle e da garantia da obtenção de boas propriedades no domínio da frequência, a sua aplicação no controle de vibrações de estruturas é limitada.

Da teoria de controle ótimo, em particular do regulador linear quadrático, notase que a lei de controle necessária para minimizar a função custo quadrática necessita que sejam medidos ou estimados todos os estados do sistema. A medição de todos os estados implica na utilização de um grande número de sensores, o que na maioria das vezes é impraticável, e para algumas estruturas a medição de alguns estados pode ser até impossível (CAI; LIM, 2005). A técnica de estimar os estados através de um observador linear também possui seus contratempos. Como o observador de estados possui sua própria dinâmica, esta técnica aumenta a complexidade do sistema e sua implementação demanda a utilização de um sistema embarcado para realizar os cálculos da dinâmica do observador, o que pode implicar em atrasos temporais na implementação do controle. Ademais, um observador de estados também é dependente do modelo da estrutura, o que pode adicionar erros ao sistema de controle.

Devido a estas dificuldades, no fim da década de 60 começaram a surgir alguns estudos sobre a minimização da função custo quadrática com restrição de realimentação da saída. Posteriormente, investigaram-se maneiras de projetar a matriz de saída, ou seja, determinar a quantidade, o tipo e a posição dos sensores. Na próxima seção, contempla-se o desenvolvimento de ambos os tópicos.

\subsection{Revisão Bibliográfica}

Esta seção descreve o progresso das pesquisas na área de controle ótimo com realimentação da saída e suas aplicações no controle de vibrações e posicionamento de 
sensores e atuadores. O escopo desta revisão será o desenvolvimento desta teoria para sistemas lineares e invariantes no tempo (LIT) contínuo. Para sistemas discretos e estocásticos recomenda-se o artigo de revisão de Mäkilä e Toivonen (MAKILA; TOIVONEN, 1987).

\subsubsection{Controle Ótimo com Realimentação da Saída}

O primeiro trabalho publicado com resultados sobre a teoria de controle ótimo com realimentação da saída para sistemas LIT foi a tese de doutorado de William Levine, de 1969 (LEVINE, 1969). Neste trabalho, Levine estabeleceu as condições necessárias para a determinação de uma lei de controle que levasse em consideração somente os estados medidos, além de propor um método numérico para solução destas condições. Estes resultados chegaram ao meio científico a partir de um artigo publicado em conjunto com seu orientador em 1970 (LEVINE; ATHANS, 1970). Neste artigo, notou-se que o problema de realimentação da saída trazia desafios além dos encontrados no problema de realimentação total de estados. Em primeiro lugar, para a determinação da lei de controle era necessária a solução de um sistema de três equações matriciais. Além disso, uma destas equações era dependente da condição inicial do sistema, o que implicava na determinação de diferentes leis de controle para diferentes condições iniciais. Por último, ainda não haviam sido investigadas as condições de existência e unicidade da solução do sistema de equações matriciais.

No artigo de Levine e Athans dois destes problemas foram abordados. Para o caso da dependência na condição inicial do sistema, sugeriu-se trocar o funcional quadrático tradicional por seu valor esperado. Assim, caso a condição inicial do sistema fosse tratada como um vetor de variáveis aleatórias com distribuição conhecida, a variável dependente da condição inicial no sistema de equações matriciais poderia ser substituída por uma matriz de covariância. Apesar de raramente se conhecer a configuração inicial de um sistema em um problema prático, esta solução foi adotada posteriormente em vários trabalhos devido a sua funcionalidade. Em relação ao sistema de equações matriciais, propôs-se um método numérico para obter sua solução. Mostrou-se que este método produzia uma sequência limitada de ganhos que sempre reduzia o valor da função custo. No entanto, não foi provada a sua convergência para um ponto ótimo.

Posteriormente, Anderson e Moore (ANDERSON; MOORE, 1971) também propuseram um algoritmo para a solução destas equações, com o mesmo problema de não provar sua convergência. A vantagem deste em relação ao mencionado anteriormente se dava pela solução de um sistema de equações de Sylvester, as quais já foram amplamente investigadas e cuja solução analítica é conhecida (BELLMAN, 1997). Alguns estudos subsequentes consideraram métodos de otimização gerais, como o método dos gradientes conjugados (HORISBERGER; BELANGER, 1974) e o método DFP (CHOI; SIRISENA, 1974). Con- 
tudo, ainda havia interesse nos métodos de Anderson-Moore e Levine-Athans por serem eficientes e atingirem o critério de parada em poucas iterações. Somente em 1985, nas pesquisas independentes de Toivonen (TOIVONEN, 1985) e Moerder e Calise (MOERDER; CALISE, 1985), foram propostas mudanças em ambos os métodos através da inserção de parâmetros de passo, mostrando que estes poderiam ser encarados como métodos de direção descendente, garantindo a sua convergência. No artigo de (TOIVONEN; MAKILA, 1985), o método modificado de Anderson-Moore é comparado favoravelmente em relação a métodos gerais de otimização, como DFP e BFGS.

A partir das propostas de algoritmos de Toivonen e Moerder-Calise foram encontradas poucas pesquisas cujo propósito era desenvolver um algoritmo para o cálculo de um ganho ótimo com realimentação de saída. Em (RAUTERT; SACHS, 1997) propõe-se um método quase-Newton com modificações específicas para o problema de realimentação de saída e mostra-se através de dois exemplos que este método pode convergir mais rápido e com uma quantidade menor de iterações que o algoritmo de Moerder-Calise. Posteriormente, em (LEIBFRITZ; MOSTAFA, 2003) foram propostos dois métodos baseados em regiões de confiança. Um destes métodos desconsiderava restrições, enquanto o outro era uma variação do método SQP com restrição. Estes algoritmos foram comparados com o proposto em (RAUTERT; SACHS, 1997) através de cinco exemplos, sendo que em quatro deles o método baseado no algoritmo SQP obteve o melhor desempenho, enquanto no outro os algoritmos obtiveram desempenho semelhante. $\mathrm{O}$ artigo mais recente encontrado que ainda tratava da proposta de um método numérico data de 2012 (ALIEV et al., 2012). Contudo, diferentemente dos outros trabalhos mencionados, o foco deste trabalho não é o desempenho do algoritmo, mas sim uma proposta de método numérico em que possam ser usadas variáveis simbólicas de modo a obter uma precisão maior nos resultados. Os artigos encontrados indicam que métodos de otimização geral mais recentes como SQP ou o método do ponto interior possuem bom desempenho para o problema de controle ótimo com realimentação de saída. Não obstante, em pesquisas recentes de aplicações dos métodos numéricos encontram-se os algoritmos de Moerder-Calise (CHANDIRAMANI, 2016), algortimo genético (GHARIB; OMRAN; EL-BAYOUMI, 2013) e SQP (DARIVANDI; MORRIS; KHAJEPOUR, 2013), o que sugere que estes algoritmos possuem desempenho satisfatório.

Em relação à questão de unicidade da solução, em (CHOI; SIRISENA, 1974) já se supunha que o problema estudado era não convexo, em consequência da dependência da solução em relação à condição inicial do algoritmo. Em 1997, Rautert e Sachs (RAUTERT; SACHS, 1997) mostraram que a Hessiana da função custo continha termos indefinidos, o que pode implicar na não convexidade. No caso da existência da solução, no artigo de Toivonen (TOIVONEN, 1985) demonstrou-se que as condições necessárias e suficientes para a existência de um controlador ótimo com realimentação da saída eram dadas pela ponderação dos modos instáveis na função custo e pela existência de um ganho constante 
de saída que estabilizasse o sistema. Este último caso representa um dos problemas mais difíceis da teoria de controle linear (POLYAK; SHCHERBAKOV, 2005), que segundo o recente artigo de revisão (SADABADI; PEAUCELLE, 2016), ainda não possui solução exata.

O problema de determinação de um ganho de saída constante que estabilize um sistema LIT começou a ser estudado no fim da década de 60. Inicialmente, além da estabilização do sistema também se investigava sobre as condições para alocação de polos. Este último problema teve sua solução publicada em (BROCKETT; BYRNES, 1981), no qual foram determinadas as condições suficientes e necessárias, baseadas somente em propriedades do sistema, para alocação arbitrária de polos com realimentação estática da saída. Para o problema de estabilização foram elaboradas diversas condições suficientes e necessárias como as apresentadas em (SYRMOS et al., 1997) e (SADABADI; PEAUCELLE, 2016), porém, nenhuma delas foi considerada satisfatória. Para o caso de um sistema LIT qualquer, nenhuma das condições apresentadas era baseada somente em propriedades do sistema, recorrendo sempre a suposição da existência de novas variáveis. Uma das dificuldades encontradas nesta abordagem estava no fato de não se obter algoritmos com garantia de convergência para uma solução, ou cuja não convergência implicasse na inexistência de solução.

Apesar de ainda não existir uma solução definitiva, algumas estratégias principais para o problema podem ser destacadas. A primeira delas consiste em tratar a estabilização a partir do método direto de Lyapunov, que implica na determinação de condições expressas através de BMIs. Estas BMIs podem ser resolvidas através dos métodos analisados em (SADABADI; PEAUCELLE, 2016) ou através de alguns pacotes computacionais como PENLAB (FIALA; KOčVARA; STINGL, 2013). A outra abordagem é caracterizada pela minimização da abcissa espectral da matriz de malha fechada. Por ser uma otimização não diferenciável e não convexa, pode-se utilizar o pacote HIFOO, que é baseado nos artigos (BURKE et al., 2006) e (BURKE; LEWIS; OVERTON, 2005). Vale ressaltar que ambos os casos não possuem garantia de convergência e que a solução possui grande dependência da condição inicial utilizada.

\subsubsection{Aplicações no Controle de Vibrações em Estruturas e Posicionamento de Sensores e Atuadores}

É provável que as aplicações da teoria controle ótimo com realimentação da saída para o controle de vibrações tenham se iniciado na década de 90 . O primeiro artigo encontrado tratando deste tema data de 1992 (HANAGUD; OBAL; CALISE, 1992). Neste trabalho, analisou-se uma viga engastada modelada através de elementos finitos com dois atuadores e sensores piezoelétricos, para os quais somente a medida de velocidade foi considerada. Posteriormente, utilizaram-se dados experimentais para estimar os fatores 
de amortecimento da viga e o modelo final utilizado consistia em uma redução do inicial a cinco modos. Essencialmente, a contribuição deste trabalho foi uma análise das ponderações das matrizes da função custo. Das diferentes propostas de ponderação adotadas, concluiu-se que a utilização de matrizes identidade resultavam em controladores que pouco aumentavam o amortecimento do sistema, sugerindo-se a utilização de uma matriz diagonal cujos elementos são proporcionais ao inverso dos quadrados dos autovalores. A questão do posicionamento dos sensores e atuadores foi mencionada como um problema extenso à parte, optando-se pela configuração colocalizada pela garantia de estabilidade. Para o vetor de estados inicial, adotou-se a proposta de Levine e Athans, considerando que este era uma variável aleatória uniformemente distribuída em uma esfera de raio unitário. Em relação a obtenção da lei de controle, foi utilizado o método proposto por Moerder e Calise, sem qualquer menção à questão de ótimos locais.

No ano seguinte, em (CHUNG; LIN; CHU, 1993) foram analisados modelos com um e três graus de liberdade, considerando sempre a realimentação de velocidade. $\mathrm{O}$ progresso em relação ao trabalho anterior se deu pela utilização de várias configurações de sensores e atuadores no caso de múltiplos graus de liberdade. Foram comparados os valores das funções custo e valores máximos de cada estado para uma entrada de aceleração na base. Concluiu-se que a realimentação com três sensores de velocidade possuía um desempenho próximo à realimentação total de estados. Para o caso de medida de somente uma velocidade, notou-se que a configuração colocalizada possuía uma desempenho melhor que o não colocalizado no que se refere aos valores de amortecimento do sistema em malha fechada. No entanto, tanto nos valores de pico dos estados quanto na magnitude da função custo, o caso não colocalizado obteve vantagem.

Posteriormente, em (FRISWELL; INMAN, 1999), mostrou-se que o controlador PPF proposto em (GOH; CAUGHEY, 1985) poderia ser projetado através das técnicas de controle ótimo com realimentação da saída. O controle PPF foi proposto como um método de redução de vibrações insensível ao fenômeno de spillover. Apesar de se tratar de um controlador dinâmico, que não está sendo considerado nesta revisão devido às questões de maior complexidade em sua implantação, é interessante considerar este trabalho devido à avaliação das questões mencionadas na subseção 1.1.1. No caso da condição inicial do algoritmo de Moerder-Calise, propõe-se a utilização de um ganho nulo para o caso de estruturas amortecidas, sem avaliar qual o impacto da utilização de outras condições na obtenção do controlador final. Para a condição inicial do sistema, também sem demonstrar matematicamente ou através de simulações, alega-se que a utilização de diferentes condições implica na obtenção de controladores ligeiramente diferentes, sendo utilizada no trabalho uma pertubação do deslocamento.

Em seguida, em (ZHOU et al., 2001), realizou-se um estudo simultâneo do controle e projeto da estrutura de um manipulador flexível. Considerou-se tanto uma otimização 
da geometria do manipulador flexível, sujeito a restrição de massa, quanto dos ganhos do controlador com realimentação da saída, considerando um critério de desempenho quadrático e o número e posição dos sensores e atuadores predeterminados. A estratégia empregada neste trabalho consistia na formulação de um loop, no qual primeiro era realizada a otimização da geometria através do algoritmo de busca aleatória IHR (ZABINSKY et al., 1993) e posteriormente a otimização dos ganhos de saída do controlador através do algoritmo de Moerder-Calise. Mostrou-se que para diferentes ponderações das matrizes da função custo obtinham-se diferentes geometrias, que possuíam em comum a redução das dimensões nas extremidades. Os problemas do algoritmo de Moerder-Calise não foram analisados, e ainda concluiu-se que o ótimo global foi obtido devido a utilização de vários parâmetros de otimização, o que é passível de indagações, já que o problema de controle ótimo com realimentação da saída é não convexo e o algoritmo de Moerder-Calise é um método de direção descendente.

Alguns outros trabalhos que consideram a aplicação da teoria de controle ótimo com realimentação da saída podem ser mencionados como (MOON, 2006), (CHANDIRAMANI, 2016) e (GHARIB; OMRAN; EL-BAYOUMI, 2013). No entanto, pouco se acrescenta na discussão dos problemas considerados anteriormente. No primeiro, trata-se do problema da localização dos sensores e atuadores através da maximização das forças e dos sinais medidos, obtendo as posições ótimas através do algoritmo genético. No segundo, o controle ótimo com realimentação da saída é comparado favoravelmente ao LQG para o controle de uma estrutura sujeita a excitações de base e laterais. No último é realizada uma comparação entre o LQR e o controle ótimo com realimentação de saída considerando a saturação do atuador, e mostra-se através de um exemplo que a partir dessa restrição o LQR leva à instabilização do sistema, enquanto a outra técnica mantém o sistema estável.

Conforme já mencionado e analisado em alguns dos trabalhos expostos anteriormente, a localização dos sensores e atuadores pode exercer influência notável sobre o desempenho do sistema de controle. Da mesma maneira como na obtenção da lei controle, uma das formas de obter a posição destes é através da minimização de critérios de desempenho. Segundo o artigo de revisão (GUPTA; SHARMA; THAKUR, 2010), os principais critérios empregados são: maximização das forças e momentos modais aplicados pelos atuadores, maximização da deflexão da estrutura, minimização do esforço de controle e maximização da dissipação de energia, maximização do grau de controlabilidade, maximização do grau de observabilidade e minimização dos efeitos de spillover. Para o terceiro critério, que foi examinado anteriormente para o projeto de um controlador com realimentação da saída, será feita uma breve análise dos principais trabalhos que também consideraram como variáveis de otimização a posição dos sensores e atuadores.

Inicialmente, destaca-se o artigo de (DEMETRIOU, 2000). O foco deste trabalho é 
a proposição de uma metodologia para a obtenção do ganho ótimo com realimentação de saída e das posições ótimas dos sensores e atuadores, simultaneamente. Esta metodologia é analisada através de um exemplo, que envolve o controle de uma viga engastada-livre. A ideia principal do trabalho é a representação das matrizes de entrada e saída do sistema como funções das posições dos sensores e atuadores. Apesar de não especificar os tipos de funções, a aplicação do método de Galerkin para o modelo da viga parece indicar um interesse na utilização de funções diferenciáveis. Retratar a dependência através desta classe de funções pode proporcionar a utilização de uma categoria mais abrangente de métodos de otimização, além de ser compatível com diversos métodos de modelagem de estruturas flexíveis. No controle de vibração da viga, apesar de não mencionar quais as ponderações utilizadas para a função custo nem o método numérico para obter as posições ótimas dos sensores e atuadores, chega-se a conclusão de que a posição ótima para o atuador piezoelétrico é na base da viga e para os dois sensores pontuais são em torno de um e dois terços da extremidade. Esta configuração com realimentação parcial de estados é comparada com a realimentação total de estados alterando-se a posição do atuador para uma vizinhança do centro da viga, concluindo-se que aquela pode proporcionar desempenhos melhores.

No mesmo ano, em (ABDULLAH, 2000), avaliou-se o posicionamento de sensores e atuadores colocalizados também para uma viga engastada-livre. Apesar de usar um modelo contínuo para este sistema, o objetivo neste caso era obter as localizações ótimas considerando um conjunto discreto de possíveis posições para os dispositivos de controle. A metodologia proposta neste artigo revelou-se um tanto intricada, pois apesar de considerar um conjunto discreto de posições, o autor utiliza o algoritmo DFP para a otimização. Além disso, a determinação das posições ótimas é realizada para cada par colocalizado por vez e após este processo efetua-se a otimização dos ganhos. A avaliação do controlador obtido é realizada através de uma análise em frequência, cuja entrada simula a atuação do vento em uma estrutura, com um espectro de força determinado. Considera-se a utilização de seis pares colocalizados com realimentação de velocidade para o controle de uma viga discretizada em sete modos. Mostrou-se que o controlador projetado reduzia a densidade espectral de posição para os primeiros modos e de aceleração para todos os modos considerados.

A maioria dos trabalhos encontrados utilizam uma metodologia semelhante a dos trabalhos analisados anteriormente, com diferenças apenas na estrutura aplicada, método de modelagem ou algoritmo de otimização. Como exemplo pode se citar (KUMAR; NARAYANAN, 2008), que estuda vigas engastada-livre e duplamente engastada modeladas através de elementos finitos e com algoritmo genético para encontrar a posição dos sensores e atuadores. Outro exemplo é (DARIVANDI; MORRIS; KHAJEPOUR, 2013), em que estudam-se uma viga duplamente apoiada e uma placa engastada em um dos lados modeladas através de elementos finitos e com algoritmo de otimização SQP. Exceto pelo 
trabalho de (FRISWELL; INMAN, 1999), todos os trabalhos mencionados consideraram a sugestão de Levine e Athans para lidar com a dependência do controlador em relação à condição inicial do sistema. Um outro exemplo que também considera esta sugestão, mas apresenta uma metodologia diferente para o posicionamento dos sensores é dado em (CAI; LIM, 2005).

A metodologia proposta em (CAI; LIM, 2005) pode ser dividida em dois passos. Inicialmente, propõe-se um método para a escolha dos estados que serão medidos e em sequência, utiliza-se a teoria de controle ótimo com realimentação da saída para projetar o controlador. A escolha dos estados que serão medidos é baseada na sensibilidade do critério de desempenho em relação ao ganho de controle, a qual é dada pela expansão em série de Taylor do critério em função do ganho. Os termos que envolvem a derivada de ordem $n$ desta expansão são denominados sensibilidade de $n$-ésima ordem. No caso da avaliação desta série no ponto de ganho ótimo, a sensibilidade de primeira ordem é nula. Portanto, para medir qual o impacto de um estado no critério de desempenho, os autores sugeriram avaliar qual a variação na sensibilidade de segunda ordem quando o ganho deste estado era anulado. No entanto, podem-se identificar algumas limitações nessa sugestão. A expansão em série de Taylor pode representar bem uma função analítica em torno do ponto no qual se realiza a expansão. Caso o ganho de um determinado estado possua um valor elevado, a variação da função anulando este termo pode ter grande influência de termos de ordem mais alta. Além disso, quando se anula um dos ganhos para um estado mantendo-se os outros, o controlador obtido não representa o ótimo com restrição de realimentação. Portanto, pode existir uma configuração cuja magnitude da função custo correspondente possa ser próxima do ótimo global caso sejam utilizados outros valores de ganho para os estados medidos. O método proposto foi aplicado para o controle com realimentação parcial de estados de modelos com três e oito graus de liberdade e foram obtidas respostas equivalentes a do controle LQR considerando como métricas o máximo deslocamento e a máxima aceleração de cada estado.

\subsection{Justificativa}

Conforme analisado nas seções anteriores, há diversas maneiras de se projetar um sistema de controle para atenuar ou mitigar vibrações em uma estrutura. No caso de um sistema de controle ativo que é projetado através da minimização de um critério de desempenho quadrático, entende-se que além do ganho de controle, as posições dos sensores e atuadores também afetam este índice de desempenho e, a partir desta perspectiva, também podem ser consideradas como variáveis de projeto. No caso do problema de controle ótimo com realimentação da saída, pôde-se concluir que a determinação das condições necessárias para a minimização dos critérios de desempenho quadráticos contribuiu para elucidar as questões de existência, unicidade e dependência do ganho de controle com 
relação à condição inicial do sistema. Logo, uma investigação sobre essas condições para o caso em que as posições dos sensores e atuadores também são incluídas como variáveis faz-se necessária devido aos seus potenciais esclarescimentos sobre o problema. Além disso, conforme o artigo (DEMETRIOU, 2000), um controlador ótimo com realimentação total de estados pode possuir um desempenho inferior a um com realimentação de saída caso os sensores e atuadores estejam bem posicionados, reforçando a importância deste estudo.

Além disso, de acordo com a revisão realizada, somente um dos artigos (FRISWELL; INMAN, 1999) não considerou a proposta dada em (LEVINE; ATHANS, 1970) para lidar com a dependência do ganho de controle com relação à condição inicial. Não obstante, neste trabalho menciona-se que a utilização de diferentes condições implica na obtenção de controladores ligeiramente diferentes, mas sem demonstrar matematicamente ou através de simulações. Portanto, entende-se que as questões sobre como a condição inicial do algoritmo de otimização ou do sistema dinâmico afetam o controlador obtido ou seu desempenho precisam ser melhor avaliadas. Um outro ponto importante a ser considerado é a questão da estabilização do sistema no caso de sensores e atuadores não colocalizados.

Por fim, pelo fato de normalmente se utilizarem critérios de desempenho baseados em coordenadas modais, um único sensor pode medir vários estados do sistema, o que pode implicar na obtenção de um controlador ótimo com realimentação total de estados. Logo, acredita-se que um estudo sobre possíveis soluções do problema do regulador linear quadrático com um número reduzido de sensores também deve ser realizado.

\subsection{Objetivos}

O objetivo deste trabalho é a proposição de uma metodologia para o projeto de um sistema de controle com realimentação de saída de forma a otimizar um critério de desempenho quadrático. Como variáveis de projeto foram incluídos parâmetros do sistema de controle que afetam o critério de desempenho, como as posições dos atuadores, posições dos sensores e ganho de controle. A princípio, são determinadas as condições necessárias para otimização da função custo considerando sistemas contínuos, ou seja, que possuem teoricamente um número infinito de posições possíveis. A partir da determinação destas condições, estudam-se os principais desafios presentes neste técnica, como a dependência das condições iniciais do sistema dinâmico e existência de um controle com realimentação de saída que estabiliza o sistema. Este estudo culmina na proposição de uma metodologia, que consiste no projeto das posições dos atuadores para um controle ótimo com realimentação total de estados e, posteriormente, a determinação das posições dos sensores e ganho de realimentação do sinal medido de modo a obter um desempenho próximo do controle ótimo para qualquer condição inicial do sistema dinâmico. A meto- 
dologia proposta é avaliada a partir de aplicações em sistemas estruturais, cujos modelos foram obtidos através do método de elementos finitos. Por último, avaliam-se as questões de estabilidade e desempenho a partir da variação de alguns parâmetros do modelo e do sistema de controle. 



\section{Tópicos da Teoria de Controle Ótimo e Di- nâmica de Estruturas}

Neste capítulo pretende-se fazer uma revisão dos principais conceitos que serão utilizados no desenvolvimento deste trabalho. Na seção de dinâmica estrutural, utilizam-se operadores diferenciais para a descrição das equações do movimento, de modo a abranger uma classe relevante de sistemas estruturais. Em seguida, faz-se uma breve revisão sobre métodos de discretização em séries para soluções de problemas de valores de contorno. Por fim, a seção sobre o regulador linear quadrático trata da obtenção do controlador ótimo, além de trazer uma breve revisão de suas propriedades e do problema de controle ótimo inverso.

\subsection{Dinâmica Estrutural}

O desenvolvimento apresentado a seguir é baseado em (MEIROVITCH, 1997) e (COURANT; HILBERT, 2004). No caso de sistemas de parâmetros distribuídos conservativos, pode-se adotar a seguinte expressão para a descrição das equações do movimento:

$$
\rho(\boldsymbol{x}) \ddot{w}(\boldsymbol{x}, t)+L w(\boldsymbol{x}, t)=f(\boldsymbol{x}, t)
$$

em que $\rho$ representa a densidade do material, $w$ o deslocamento transversal, $\boldsymbol{x}$ o vetor de coordenadas que descreve o domínio da estrutura, $f$ o carregamento distribuído e $L$ um operador linear diferencial da seguinte forma:

$$
L=a+b_{i} \frac{\partial}{\partial x_{i}}+c_{i j} \frac{\partial^{2}}{\partial x_{i} \partial x_{j}}+\ldots
$$

sendo $c_{i j}$ simétrico. A notação de Einstein foi utilizada para simplificar os somatórios. Os termos $a, b_{i}, \ldots$ são funções conhecidas da variável espacial $\boldsymbol{x}$. Assume-se que o operador $L$ envolva derivadas de ordem até $2 p$, sendo $p$ um inteiro. A partir destas considerações, é possível encontrar uma solução para a equação (2.1) que seja separável nas variáveis do espaço e do tempo, ou seja, em que cada ponto do sistema execute o mesmo movimento no tempo. Matematicamente, esta afirmação pode ser expressa como:

$$
w(\boldsymbol{x}, t)=\phi(\boldsymbol{x}) F(t)
$$


Considerando a ausência de forças externas e substituindo (2.3) em (2.1), obtém-se a seguinte equação:

$$
-\frac{L \phi}{\rho \phi}=\frac{\ddot{F}}{F}
$$

como o lado esquerdo da equação depende somente de $\boldsymbol{x}$ e o lado direito somente de $t$ e ambas as variáveis são independentes, os dois lados da equação devem ser iguais a uma constante. Ademais, como ambos os lados são compostos por funções reais, esta constante também deve ser real. Logo, a constante será denotada por $-\lambda$, sendo $\lambda$ um número positivo. Igualando o lado direito de (2.4) a $-\lambda$, obtém-se uma equação diferencial ordinária de segunda ordem, a qual pode ser facilmente resolvida. No caso do lado esquerdo, chega-se na seguinte expressão:

$$
L \phi=\lambda \rho \phi
$$

a qual representa uma equação diferencial parcial de ordem $2 p$. Em conjunto com a equação (2.5), devem existir $p$ condições de contorno para cada ponto da fronteira do domínio $D$ definido por $\boldsymbol{x}$ :

$$
B_{i} \phi=0 \quad i=1,2, \ldots, p
$$

em que cada $B_{i}$ representa um operador linear diferencial de ordem no máximo $2 p-1$. O problema dado pela equação (2.5) sujeita às restrições dadas por (2.6) é denominado problema diferencial de autovalor, e consiste em determinar os valores do parâmetro $\lambda$ para os quais existem funções não triviais $\phi$ que satisfazem ambas as equações. Este problema admite como solução um conjunto infinito e contável de parâmetros $\lambda_{i}$ e funções $\phi_{i}$, os quais são chamados de autovalores e autofunções, respectivamente.

Para se determinar algumas propriedades da solução do problema diferencial de autovalor, deve-se introduzir o conceito de operador autoadjunto. Dado um espaço de Hilbert de funções reais definidas em um domínio $D$, define-se o produto interno de dois de seus elementos $f$ e $g$ como:

$$
(f, g)=\int_{D} f g d D
$$

Neste caso, um operador $L$ é autoadjunto se satisfaz a seguinte propriedade:

$$
(f, L g)=(g, L f)
$$

Para o caso em que o operador dado na equação (2.5) satisfaz esta condição, é possível extrair algumas propriedades dos autovalores e autofunções. Dadas duas solu- 
ções distintas do problema de autovalor $\left(\lambda_{r}, \phi_{r}\right)$ e $\left(\lambda_{s}, \phi_{s}\right)$, pode-se escrever as seguintes equações:

$$
\begin{aligned}
& L \phi_{r}=\lambda_{r} \rho \phi_{r} \\
& L \phi_{s}=\lambda_{s} \rho \phi_{s}
\end{aligned}
$$

multiplicando a primeira por $\phi_{s}$ e a segunda por $\phi_{r}$, subtraindo a segunda da primeira e integrando no domínio $D$, obtém-se:

$$
\int_{D}\left(\phi_{s} L \phi_{r}-\phi_{r} L \phi_{s}\right) d D=\left(\lambda_{r}-\lambda_{s}\right) \int_{D} \rho \phi_{r} \phi_{s} d D
$$

mas como o operador $L$ é autoadjunto e os autovalores $\lambda_{r}$ e $\lambda_{s}$ são distintos, a equação (2.10) pode ser reescrita como:

$$
\int_{D} \rho \phi_{r} \phi_{s} d D=0
$$

a qual representa a relação de ortogonalidade das autofunções com relação à função de densidade $\rho(\boldsymbol{x})$. Multiplicando a equação $(2.9 \mathrm{~b})$ por $\phi_{r}$, integrando sobre $D$ e usando a relação de ortogonalidade obtida anteriormente obtém-se a segunda relação de ortogonalidade:

$$
\int_{D} \phi_{r} L \phi_{s} d D=0
$$

No caso de um autovalor repetido, o número de funções correspondentes a este autovalor é igual a sua multiplicidade. Apesar destas funções não serem ortogonais, elas são independentes, de modo que é possível torná-las ortogonais através do método de ortogonalização de Gram-Schmidt. Assim, todas as funções de um sistema autoadjunto podem ser consideradas ortogonais. Como o problema de autovalor considerado é homogêneo, somente a forma das funções pode ser determinada, de modo que sua amplitude é arbitrária. Utilizando a relações de ortogonalidade das autofunções, estas podem ser normalizadas de acordo com as seguintes equações:

$$
\int_{D} \rho \phi_{r} \phi_{s} d D=\delta_{r s} \quad r, s=1,2, \ldots
$$

o que implica em:

$$
\int_{D} \phi_{r} L \phi_{s} d D=\lambda_{r} \delta_{r s} \quad r, s=1,2, \ldots
$$

em que $\delta_{r s}$ representa o delta de Kronecker. 
Os resultados obtidos até o momento revelam algumas propriedades da solução da equação (2.1) no caso da ausência de forças externas. No entanto, de acordo com o teorema da expansão para sistemas autoadjuntos, algumas propriedades obtidas até o momento podem ser estendidas para o caso de um problema não homogêneo. Este teorema mostra que qualquer função $f$ que satisfaça as condições de contorno (2.6) e possui $L f$ contínuo, pode ser expandida em uma série absolutamente e uniformemente convergente das autofunções:

$$
f=\sum_{i=1}^{\infty} c_{i} \phi_{i}
$$

com os coeficientes $c_{i}$ dados por:

$$
c_{i}=\int_{D} \rho \phi_{i} f d D
$$

Logo, qualquer solução separável nas variáveis do tempo e espaço que satisfaça a equação (2.1) deve ser da forma:

$$
w(\boldsymbol{x}, t)=\sum_{i=1}^{\infty} \eta_{i}(t) \phi_{i}(\boldsymbol{x})
$$

em que as funções temporais $\eta_{i}(t)$ assumem o papel dos coeficientes $c_{i}$, e são denominadas coordenadas modais. Este resultado pode ser utilizado para transformar o problema de equações diferenciais parciais do movimento de um corpo flexível em um sistema de infinitas equações diferenciais ordinárias independentes. Substituindo $w$ dado na equação (2.17) em (2.1), multiplicando esta por $\phi_{r}$ e integrando ambos os lados no domínio $D$, chega-se no seguinte sistema de equação diferenciais ordinárias independentes:

$$
\ddot{\eta}_{r}(t)+\lambda_{r} \eta_{r}(t)=N_{r}(t) \quad r=1,2, \ldots
$$

$\operatorname{com} N_{r}(t)$ dado por:

$$
N_{r}(t)=\int_{D} \phi_{r}(\boldsymbol{x}) f(\boldsymbol{x}, t) d D \quad r=1,2, \ldots
$$

No caso de sistemas que possuem amortecimento viscoso, a força de amortecimento pode ser tratada como um carregamento distribuído proporcional à velocidade:

$$
\rho(\boldsymbol{x}) \ddot{w}(\boldsymbol{x}, t)+L w(\boldsymbol{x}, t)=-C \dot{w}(\boldsymbol{x}, t)+f(\boldsymbol{x}, t)
$$

Utilizando o mesmo procedimento para obtenção da equação (2.18), chega-se nas seguinte equações: 


$$
\begin{gathered}
\ddot{\eta}_{r}(t)+\sum_{s=1}^{\infty} c_{r s} \dot{\eta}_{s}(t)+\lambda_{r} \eta_{r}(t)=N_{r}(t) \quad r=1,2, \ldots \\
c_{r s}=\int_{D} \phi_{r}(\boldsymbol{x}) C \phi_{s}(\boldsymbol{x}) d D \quad r, s=1,2, \ldots
\end{gathered}
$$

que representam um sistema infinito de equações diferenciais acopladas. Usualmente, adota-se o caso em que o operador $C$ pode ser expresso como uma combinação linear de $\rho$ e $L$, de modo que também seja possível usá-lo nas relações de ortogonalidade. Assim, o termo $c_{r s}$ pode ser dado por:

$$
c_{r s}=2 \zeta_{r} \omega_{r} \delta_{r s}, \quad \omega_{r}^{2}=\lambda
$$

sendo cada $\omega_{r}$ denominado frequência natural do sistema. Assim, o sistema de equações acopladas pode ser reescrito como um sistema de equações diferenciais ordinárias de segundo grau desacopladas:

$$
\ddot{\eta}_{r}(t)+2 \zeta_{r} \omega_{r} \dot{\eta}_{s}+\omega_{r}^{2} \eta_{r}(t)=N_{r}(t) \quad r=1,2, \ldots
$$

Deve-se ressaltar que para obter o sistema de equações diferenciais ordinárias é necessário resolver o problema diferencial de autovalor, o qual possui solução exata somente em alguns casos. No entanto, existem alguns métodos para aproximar a solução deste problemas, os quais podem ser divididos em métodos de parâmetros concentrados e métodos de discretização em séries. Os métodos de parâmetros concentrados consistem em discretizar a massa distribuída do sistema em alguns pontos e tratar a rigidez do sistema através de coeficientes de influência de rigidez ou também através de discretização. Como exemplo de métodos de parâmetros concentrados pode se citar os métodos de Holzer e Myklestad. Métodos de discretização em séries consistem em aproximar as autofunções do problema diferencial de autovalor por um conjunto finito de funções conhecidas. Na seção a seguir é realizada uma breve revisão sobre estes métodos.

\subsection{Métodos de discretização em séries}

Para se obter a resposta de uma estrutura a uma determinada excitação é necessário resolver a equação do movimento (2.1). Uma maneira de resolver esse problema seria utilizar um método como o de diferenças finitas no domínio do tempo (TAFLOVE; HAGNESS, 2005), usualmente utilizado para problemas de eletromagnetismo, o qual resolve a equação diferencial parcial tanto nas variáveis do espaço quanto no tempo. No entanto, em problemas com estruturas flexíveis há algumas aplicações em que é conveniente ter um modelo no domínio do tempo ou frequência, como identificação de parâmetros e controle. Portanto, os métodos numéricos utilizados nesse tipo de problema são aqueles em 
que se elimina a dependência das variáveis espaciais. Nos métodos de discretização em séries essa dependência é eliminada através da aproximação das autofunções por um conjunto finito de funções conhecidas. A vantagem desses métodos em relação aos métodos de parâmetros concentrados se da em relação às propriedades de convergência e maior facilidade na geração de modelos computacionais. Nesta seção são apresentados os métodos de Rayleigh-Ritz, dos resíduos ponderados e de elementos finitos (FEM). Este último é apresentado como uma extensão dos outros dois métodos, e são discutidas algumas diferenças em questão de implementação do método e convergência. O desenvolvimento a seguir também é baseado em (MEIROVITCH, 1997).

Para apresentar o método de Rayleigh-Ritz, primeiro retoma-se o problema diferencial de autovalor para um operador autoadjunto $L$ :

$$
L \phi=\lambda \rho \phi
$$

Multiplicando esta equação por $\phi$ e integrando em $D$ chega-se em:

$$
\int_{D} \phi L \phi d D=\lambda \int_{D} \rho \phi^{2} d D
$$

a qual pode ser reescrita como:

$$
R(\phi)=\lambda=\omega^{2}=\frac{\int_{D} \phi L \phi d D}{\int_{D} \rho \phi^{2} d D}
$$

em que $R(\phi)$ é conhecido como quociente de Rayleigh. Como demonstrado em (MEIROVITCH, 1997), para um operador autoadjunto o quociente de Rayleigh possui valores estacionários nas autofunções do sistema, e os valores do quociente para estas autofunções são os autovalores associados. Logo, o método de Rayleigh-Ritz tenta aproximar as autofunções do problema utilizando um conjunto de funções conhecidas, chamadas de funções teste, e fazendo a variação do quociente de Rayleigh ser nula para combinações lineares destas funções. Para satisfazer o problema diferencial de autovalor, as funções teste devem ser independentes e pertencer a um conjunto completo $\left\{\psi_{1}(\boldsymbol{x}), \ldots, \psi_{n}(\boldsymbol{x}), \ldots\right\}$, além de satisfazer as condições de contorno do problema (2.6) e possuir ordem no mínimo $2 p$. Assim, utilizando um número finito $n$ de funções deste conjunto, as funções que aproximam as autofunções do problema podem ser escritas na forma:

$$
w(\boldsymbol{x})=\sum_{i=1}^{n} a_{i} \psi_{i}(\boldsymbol{x})=\boldsymbol{\psi}^{\top} \boldsymbol{a}
$$


sendo os coeficientes $a_{i}$ constantes a serem determinadas. A partir da substituição de $w$ no quociente de Rayleigh, este passa a ser função dos coeficientes $a_{i}$ :

$$
R\left(a_{1}, \ldots, a_{n}\right)=\frac{\sum_{i=1}^{n} \sum_{j=1}^{n} k_{i j} a_{i} a_{j}}{\sum_{i=1}^{n} \sum_{j=1}^{n} m_{i j} a_{i} a_{j}}
$$

em que, por $L$ ser autoadjunto,

$$
\begin{aligned}
k_{i j}=k_{j i}=\int_{D} \psi_{i} L \psi_{j} d D, & i, j=1, \ldots, n \\
m_{i j}=m_{j i}=\int_{D} \rho \psi_{i} \psi_{j} d D, & i, j=1, \ldots, n
\end{aligned}
$$

sendo $k_{i j}$ e $m_{i j}$ os coeficientes de rigidez e massa, respectivamente.

Para determinar os valores dos coeficientes $a_{i}$, faz-se a variação do quociente de Rayleigh ser nula:

$$
\delta R=\sum_{i=1}^{n} \frac{\partial R}{\partial a_{i}} \delta a_{i}=0
$$

que devido ao fato de os coeficientes $a_{i}$ serem independentes se traduz em:

$$
\frac{\partial R}{\partial a_{i}}=0, \quad i=1, \ldots, n
$$

Calculando a derivada para $a_{r}$ obtém-se:

$$
\begin{gathered}
\frac{\left(\frac{\partial}{\partial a_{r}} \sum_{i=1}^{n} \sum_{j=1}^{n} k_{i j} a_{i} a_{j}\right)\left(\sum_{i=1}^{n} \sum_{j=1}^{n} m_{i j} a_{i} a_{j}\right)-\left(\sum_{i=1}^{n} \sum_{j=1}^{n} k_{i j} a_{i} a_{j}\right)\left(\frac{\partial}{\partial a_{r}} \sum_{i=1}^{n} \sum_{j=1}^{n} m_{i j} a_{i} a_{j}\right)}{\left(\sum_{i=1}^{n} \sum_{j=1}^{n} m_{i j} a_{i} a_{j}\right)^{2}} \\
=\frac{2}{\sum_{i=1}^{n} \sum_{j=1}^{n} m_{i j} a_{i} a_{j}}\left(\sum_{j=1}^{n} k_{r j} a_{j}-\lambda \sum_{j=1}^{n} m_{r j} a_{j}\right)=0, \quad r=1, \ldots, n
\end{gathered}
$$

a qual é satisfeita se:

$$
\sum_{j=1}^{n} k_{r j} a_{j}-\lambda \sum_{j=1}^{n} m_{r j} a_{j}=0, \quad r=1, \ldots, n
$$


Esta equação ainda pode ser reescrita na forma matricial:

$$
K \boldsymbol{a}=\lambda M \boldsymbol{a}
$$

em que:

$$
\begin{aligned}
& K=\int_{D} \boldsymbol{\psi} L \boldsymbol{\psi}^{\top} d D \\
& M=\int_{D} \rho \boldsymbol{\psi} \boldsymbol{\psi}^{\top} d D
\end{aligned}
$$

mostrando que o problema de aproximar autofunções e autovalores através do método de Rayleigh-Ritz é análogo ao problema de autovalor de um sistema conservativo discreto. Cada autovalor $\lambda_{r}$ encontrado como solução do problema (2.35) aproxima os respectivos autovalores do problema contínuo e a autofunção aproximada associada é calculada substituindo o autovetor $\boldsymbol{a}_{r}$ em (2.28):

$$
w_{r}=\boldsymbol{\psi}^{\top} \boldsymbol{a}_{r}
$$

Conforme mencionado anteriormente, as funções teste devem ser de ordem no mínimo $2 p$ e satisfazer todas as condições de contorno do sistema. No entanto, este requisito pode ser suavizado através de uma modificação no quociente de Rayleigh. Considerando um sistema unidimensional por simplicidade e integrando o numerador de (2.27) por partes têm-se:

$$
\int_{0}^{L} \phi L \phi d x=[\phi, \phi]=\int_{0}^{L} \sum_{k=1}^{p} y_{k}\left(\frac{d^{k} \phi}{d x^{k}}\right)^{2} d x+\left.\sum_{l=1}^{p-1} z_{l}\left(\frac{d^{l} \phi}{d x^{l}}\right)^{2}\right|_{0} ^{L}
$$

em que os coeficientes $y_{k}$ e $z_{l}$ são funções de $x$ e a expressão $[\phi, \phi]$ é denominada produto interno de energia e representa uma medida da energia potencial do sistema. Nesta equação, nota-se que o produto interno de energia do sistema requer derivadas somente de ordem $p$ para as funções. Além disso, através da avaliação do segundo somatório de (2.38) nos pontos de fronteira, é possível especificar as condições de contorno naturais do sistema, que resultam da aplicação de forças e momentos. Desse modo, as condições de contorno naturais já estão implícitas nesta forma do quociente de Rayleigh, a qual é conhecida como forma de energia. Logo, esta forma requer que as funções teste sejam de ordem p e satisfaçam somente as condições de contorno geométricas do problema.

Por fim, faz-se uma breve análise da convergência do método de Rayleigh-Ritz. Conforme mencionado anteriormente, os autovalores do sistema são pontos estacionários do quociente de Rayleigh. Desse modo, conclui-se que o autovalor associado a frequência fundamental do sistema é um ponto de mínimo. Portanto, a aproximação deste autovalor 
$\left(\lambda_{1}\right)$ através do método de Rayleigh-Ritz sempre implicará na obtenção de um autovalor $\left(\lambda_{1}^{r}\right)$ de magnitude maior ou igual:

$$
\lambda_{1} \leq \lambda_{1}^{r}
$$

Ainda, utilizando o teorema max-min de Courant-Fischer (MEIROVITCH, 1997) é possível mostrar que esta relação é válida para qualquer autovalor calculado usando o método de Rayleigh-Ritz. Este teorema diz que para dadas funções $v_{j}$ que pertencem ao espaço de funções que satisfazem os requisitos do quociente de Rayleigh, um autovalor $\lambda_{s}$ pode ser determinado a partir da seguinte relação:

$$
\lambda_{s}=\max _{v_{j}} \min _{w} R(w), \quad\left(w, v_{j}\right)=0, \quad j=1, \ldots, s-1
$$

em que $\left(w, v_{j}\right)$ é o produto interno $(2.7)$ entre $w$ e $v_{j}$. Da mesma forma, o autovalor associado $\lambda_{s}^{r}$ calculado através do método de Rayleigh-Ritz (2.35) é dado por:

$$
\begin{array}{r}
\lambda_{s}^{r}=\max _{v_{j}} \min _{w} R(w), \quad\left(w, v_{j}\right)=0, \quad j=1, \ldots, s-1, \\
\left(w, \psi_{k}\right)=0, \quad k=n+1, n+2, \ldots
\end{array}
$$

Portanto, como o espaço de funções para calcular o autovalor aproximado $\lambda_{s}^{r}$ é apenas um subespaço do espaço para calcular $\lambda_{s}$, conclui-se que os autovalores aproximados através do método de Rayleigh-Ritz são sempre maiores ou iguais aos autovalores reais:

$$
\lambda_{s} \leq \lambda_{s}^{r}, \quad s=1, \ldots, n
$$

Outra questão importante do método de Rayleigh-Ritz é sobre como se altera a aproximação dos autovalores quando a quantidade de funções teste é modificada. Considerando a utilização de $(n+1)$ funções teste, chega-se no problema de autovalor dado pela equação $(2.35)$, com as matrizes $K^{(n+1)}$ e $M^{(n+1)}$ dadas por:

$$
K^{(n+1)}=\left[\begin{array}{c|c}
K^{(n)} & \boldsymbol{k} \\
\hline \boldsymbol{k}^{\top} & k
\end{array}\right], \quad M^{(n+1)}=\left[\begin{array}{c|c}
M^{(n)} & \boldsymbol{m} \\
\hline \boldsymbol{m}^{\top} & m
\end{array}\right],
$$

em que $K^{(n)}$ e $M^{(n)}$ são as matrizes do problema com $n$ funções teste, $\boldsymbol{k}$ e $\boldsymbol{m}$ são vetores de dimensão $n$ e $m$ e $k$ são escalares. Nota-se que as matrizes são alteradas somente pela adição de uma coluna e uma linha, sem qualquer outra modificação. Portanto, pode-se aplicar o teorema do entrelaçamento de autovalores (STRANG, 2006), que diz que os autovalores dos problema de dimensão $n$ e $(n+1)$ são relacionados da seguinte forma:

$$
\lambda_{1}^{(n+1)} \leq \lambda_{1}^{(n)} \leq \lambda_{2}^{(n+1)} \leq \lambda_{2}^{(n)} \leq \ldots \leq \lambda_{n}^{(n+1)} \leq \lambda_{n}^{(n)} \leq \lambda_{n+1}^{(n+1)}
$$


o que implica em uma melhor aproximação dos autovalores reais do sistema conforme o aumento do número de funções teste utilizadas.

O método de Rayleigh-Ritz consiste em encontrar os pontos estacionários do quociente de Rayleigh e, portanto, só pode ser aplicado a sistemas autoadjuntos. Uma alternativa para lidar com sistemas que não pertencem a esta classe é utilizar o método de resíduos ponderados. Este método também utiliza uma função da forma (2.28) para resolver o problema diferencial de autovalor. No entanto, sabendo que esta função é somente uma aproximação das autofunções, define-se um resíduo:

$$
E(w, \boldsymbol{x})=L w-\lambda \rho w
$$

o qual representa o erro para a função de aproximação $w$ em cada ponto $\boldsymbol{x}$ do domínio. De modo semelhante ao método de Rayleigh-Ritz, multiplica-se esta equação a esquerda por funções $\varphi_{i}$, obtendo o resíduo ponderado:

$$
\varphi_{i} E=\varphi_{i}(L w-\lambda \rho w), \quad i=1, \ldots, n
$$

sendo $\varphi_{i}$ as funções de ponderação do resíduo. O objetivo deste método é fazer com que o resíduo seja o menor possível para a função de aproximação $w$; portanto, integra-se a expressão anterior para cada $\varphi_{i}$ e impõe-se que o resultado seja igual a zero:

$$
\int_{D} \varphi_{i} E d D=\int_{D} \varphi_{i}(L w-\lambda \rho w) d D=0, \quad i=1, \ldots, n
$$

a qual, substituindo (2.28) para $w$ fica:

$$
\int_{D} \varphi_{i} E d D=\int_{D} \varphi_{i}\left(\sum_{j=1}^{n} a_{j} L \psi_{j}-\lambda \sum_{j=1}^{n} a_{j} \rho \psi\right) d D=0, \quad i=1, \ldots, n
$$

que substituindo o vetor $\boldsymbol{\varphi}=\left[\varphi_{1} \ldots \varphi_{n}\right]$ e os vetores $\boldsymbol{\psi}$ e $\boldsymbol{a}$ já definidos anteriormente, pode ser reescrita na forma matricial:

$$
K \boldsymbol{a}=\lambda M \boldsymbol{a}
$$

em que:

$$
\begin{aligned}
& K=\int_{D} \boldsymbol{\varphi} L \boldsymbol{\psi}^{\top} d D \\
& M=\int_{D} \rho \boldsymbol{\varphi} \boldsymbol{\psi}^{\top} d D
\end{aligned}
$$


Nota-se que através do método dos resíduos ponderados chega-se no mesmo problema algébrico de autovalor, porém, com as matrizes $K$ e $M$ dadas por expressões diferentes. No entanto, por ser baseado diretamente no problema diferencial de autovalor, o método dos resíduos ponderados também vale para sistemas não autoadjuntos. Ainda, no caso em que o operador $L$ não é autoadjunto as matrizes $K$ e $M$ podem não ser simétricas, o que pode implicar em autovalores complexos. Assim como no método de Rayleigh-Ritz, integrando-se por partes o termo que envolve $L$ chega-se a conclusão de que é possível utilizar funções de ordem $p$ que satisfazem somente as condições de fronteira geométricas. O caso particular em que as funções de ponderação são iguais às funções teste $(\boldsymbol{\varphi}=\boldsymbol{\psi})$ é conhecido como método de Galerkin, e para sistemas autoadjuntos é equivalente ao método de Rayleigh-Ritz.

O principal problema dos métodos apresentados anteriormente é a escolha de um conjunto completo de funções teste que satisfaçam as condições de contorno, que além de ser uma tarefa laboriosa não possui generalização para geometrias complexas, o que dificulta sua implementação em pacotes computacionais. Neste sentido, o método de elementos finitos possui uma grande vantagem. A principal diferença entre os métodos apresentados anteriormente e o FEM está no domínio das funções teste. Apesar de os métodos de Rayleigh-Ritz e dos resíduos ponderados não exigirem nenhuma restrição sobre o domínio das funções, tradicionalmente estes ficaram conhecidos por utilizarem funções de domínio global. No método de elementos finitos a estrutura é divida em subdomínios nos quais são definidas funções locais, e são aplicados os mesmos procedimentos dos métodos de Rayleigh-Ritz e dos resíduos ponderados localmente.

No FEM utiliza-se também uma aproximação da forma (2.28), em que as funções teste, também conhecidas como funções de interpolação, são geralmente polinômios de baixa ordem, o que facilita os cálculos de integração. Além disso, os coeficientes que multiplicam as funções de interpolação possuem um significado físico, e são associados ao deslocamento ou deflexão de alguns pontos na fronteira dos subdomínios, os quais são denominados nós. No caso de uma viga em que o subdomínio é definido conforme a Figura 1, é comum utilizar as seguintes funções de interpolação:

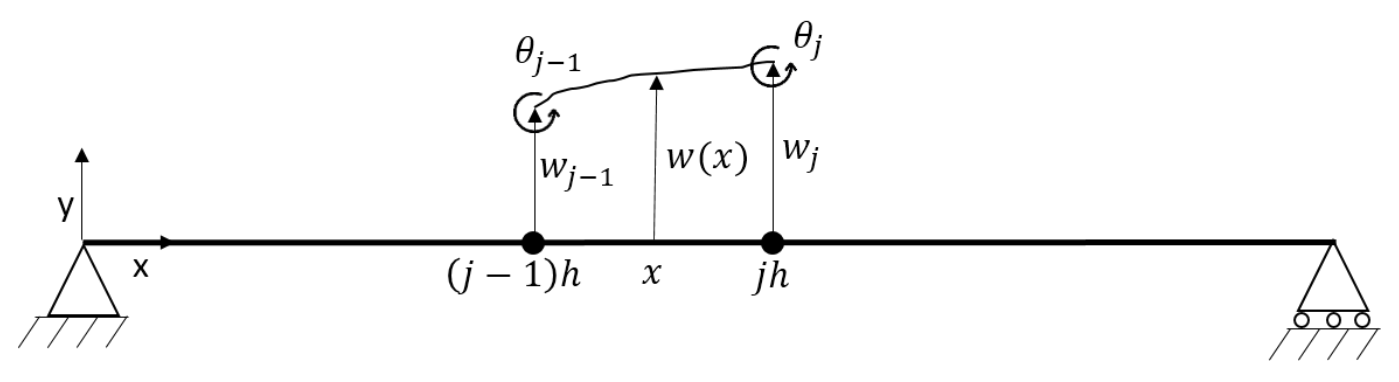

Figura 1 - Deslocamento e deflexão definidos ao longo de um elemento $j$ da viga. 


$$
\begin{aligned}
\psi_{1}=3 \mu^{2}-2 \mu^{3}, \quad \psi_{2}=\mu^{2}-\mu^{3}, \quad \psi_{3} & =1-3 \mu^{2}+2 \mu^{3}, \quad \psi_{4}=-\mu+2 \mu^{2}-\mu^{3} \\
\mu & =\frac{j h-x}{h}
\end{aligned}
$$

em que as funções $\psi_{1}, \psi_{2}, \psi_{3}$ e $\psi_{4}$ são conhecidas como polinômios de Hermite, e multiplicam $w_{j-1}, h \theta_{j-1}, w_{j}$ e $h \theta_{j}$, respectivamente. No caso de uma placa com subdomínio definido conforme a Figura 2, pode-se utilizar as seguintes funções de interpolação (REDDY, 2006):

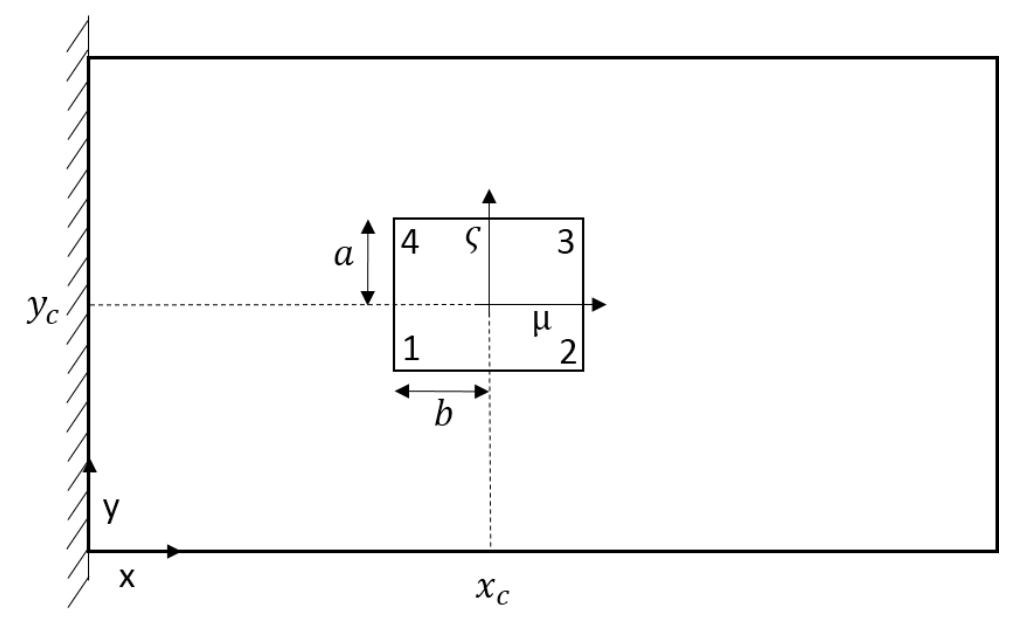

Figura 2 - Coordenadas locais e globais para um elemento retangular em uma placa.

$$
\begin{gathered}
\psi_{I}=\frac{1}{8}\left(\mu_{0}+1\right)\left(\varsigma_{0}+1\right)\left(2+\mu_{0}+\varsigma_{0}-\mu^{2}-\varsigma^{2}\right) \\
\psi_{I+1}=\frac{1}{8} \mu_{i}\left(\mu_{0}+1\right)^{2}\left(\mu_{0}-1\right)\left(\varsigma_{0}+1\right) \\
\psi_{I+2}=\frac{1}{8} \varsigma_{i}\left(\mu_{0}+1\right)\left(\varsigma_{0}-1\right)\left(\varsigma_{0}+1\right)^{2} \\
\mu=\frac{x-x_{c}}{a}, \quad \varsigma=\frac{y-y_{c}}{b}, \quad \mu_{0}=\mu \mu_{i}, \quad \varsigma_{0}=\varsigma_{i} \varsigma, \quad I=3 i-2, \quad i=1, \ldots, 4
\end{gathered}
$$

em que $i$ representa os nós do elemento, $\left(\mu_{i}, \varsigma_{i}\right)$ são os valores das coordenadas $(\mu, \varsigma)$ no nó $i, \psi_{I}, \psi_{I+1}$ e $\psi_{I+2}$ são as funções de interpolação que multiplicam $w_{i}, \frac{\partial w_{i}}{\partial x}$ e $\frac{\partial w_{i}}{\partial y}$, respectivamente.

Apesar de o FEM e os métodos de Rayleigh-Ritz e dos resíduos ponderados serem semelhantes em sua base matemática, ainda há uma diferença na questão de convergência entre ambos os métodos. No método de Rayleigh-Ritz, ao se adicionar uma função teste as matrizes de massa e rigidez se alteram somente pela adição de uma linha e coluna, de modo que é possível utilizar o teorema do entrelaçamento dos autovalores. No caso do FEM, o refinamento do modelo é realizado através da adição de nós, o que muda os domínios das funções e consequentemente altera estas matrizes de forma distinta, de forma 
que não existe prova matemática de que o teorema do entrelaçamento ainda se mantém (MEIROVITCH; BARUH, 1983). No entanto, ainda é possível provar que o método de elementos finitos converge (STRANG; FIX, 2008), mas como há diferentes formas de refinamento de malha, a demonstração neste caso é uma tarefa complicada.

\subsection{Regulador Linear Quadrático}

O problema do regulador linear quadrático (LQR) consiste em encontrar uma lei de controle para um sistema dinâmico de forma a minimizar uma função custo quadrática. $\mathrm{Na}$ literatura de controle, usualmente utilizam-se três formas de obter esta lei: através do cálculo variacional, a partir da equação de Hamilton-Jacobi-Bellman (HJB) e através de uma modificação da função custo utilizando o método direto de Lyapunov, que culmina na utilização de métodos tradicionais do cálculo. No desenvolvimento a seguir, considerase este último caso para a obtenção da lei de controle para um sistema linear e invariante no tempo (LIT). Esta formulação será particularmente interessante no capítulo seguinte, no caso da obtenção do controle com restrição de realimentação da saída. Um desenvolvimento semelhante pode ser encontrado em (LEWIS; VRABIE; SYRMOS, 2012).

Dado o sistema de equações diferenciais:

$$
\dot{\boldsymbol{x}}(t)=A \boldsymbol{x}(t)+B \boldsymbol{u}(\boldsymbol{x}, t)
$$

sendo $\boldsymbol{x} \in \mathbb{R}^{n}$ o vetor de estados, $\boldsymbol{u} \in \mathbb{R}^{a}$ o vetor de entrada, $A \in \mathbb{R}^{n \times n}$ a matriz de estados e $B \in \mathbb{R}^{n \times a}$ a matriz de entrada, tenciona-se determinar uma lei de controle proporcional aos estados $(\boldsymbol{u}=-G \boldsymbol{x})$ de forma a minimizar a seguinte função custo:

$$
J=\int_{0}^{\infty} \boldsymbol{x}^{\top} Q \boldsymbol{x}+\boldsymbol{u}^{\top} R \boldsymbol{u} d t
$$

que substituindo $\boldsymbol{u}=-G \boldsymbol{x}$ pode ser reescrita como:

$$
J=\int_{0}^{\infty} \boldsymbol{x}^{\top}\left(Q+G^{\top} R G\right) \boldsymbol{x} d t
$$

de forma que $Q$ é positiva semidefinida e $R$ é positiva definida, ambas simétricas. Estas hipóteses são necessárias para assegurar que a função custo seja sempre positiva. Notase também que todos os modos instáveis do sistema devem ser ponderados em $Q$; do contrário, é possível encontrar uma lei de controle ótima que não estabilize o sistema. Por último, o sistema deve ser estabilizável, ou seja, deve existir uma função $\boldsymbol{u}(\boldsymbol{x})$ que garanta que a origem $(\boldsymbol{x}=\mathbf{0})$ de (2.54) seja um ponto de equilíbrio estável.

A partir desta definição de sistema estabilizável, pode-se introduzir o conceito de função de controle de Lyapunov $(c l f)$. Na teoria de sitemas dinâmicos, funções de 
Lyapunov são utilizadas para determinar quando um ponto de equilíbrio é estável. No caso da teoria de controle, a existência de uma clf implica na estabilização do sistema através da realimentação total de estados (ARTSTEIN, 1983). Uma clf $V: \mathbb{R}^{n} \rightarrow \mathbb{R}$ é uma função positiva definida tal que:

$$
\forall \boldsymbol{x} \neq \mathbf{0}, \exists \boldsymbol{u} \mid \dot{V}(\boldsymbol{x})=\nabla V \frac{d \boldsymbol{x}}{d t}<0
$$

Por $V$ ser positiva definida e sua derivada no tempo ser sempre menor que zero (exceto em $\boldsymbol{x}=\mathbf{0}$ ), conclui-se que a origem é um ponto de equilíbrio estável para uma dada função de controle. No caso de sistemas lineares, é usual utilizar a seguinte função $V$ quadrática:

$$
V(\boldsymbol{x})=\boldsymbol{x}^{\top} P \boldsymbol{x}
$$

em que $P$ é uma matriz positiva definida e simétrica. Para que $V$ seja uma $c l f$ é necessário que $\dot{V}$ seja negativa definida. Portanto, utilizando o integrando da função custo (2.56), é possível adotar a seguinte expressão para $\dot{V}$ :

$$
\dot{V}=\frac{d\left(\boldsymbol{x}^{\top} P \boldsymbol{x}\right)}{d t}=-\boldsymbol{x}^{\top}\left(Q+G^{\top} R G\right) \boldsymbol{x}
$$

Expandindo o termo com a derivada temporal, utilizando a equação (2.54), adotando $\boldsymbol{u}=-G \boldsymbol{x}$ e utilizando a notação $A_{c}$ para a matriz de malha fechada $(A-B G)$, obtém-se:

$$
\boldsymbol{x}^{\top}\left(A_{c}^{\top} P+P A_{c}+Q+G^{\top} R G\right) \boldsymbol{x}=0
$$

a qual deve ser válida para qualquer valor de $\boldsymbol{x}$. Logo, conclui-se que:

$$
A_{c}^{\top} P+P A_{c}+Q+G^{\top} R G=0
$$

Esta equação é chamada de equação de Lyapunov e, dada uma matriz $G$ de modo que o sistema seja estável, sempre possui solução única. A equação de Lyapunov é um caso particular da equação de Sylvester, a qual possui solução analítica conhecida (BELLMAN, 1997). Dadas estas considerações, é possível utilizar a matriz $P$ solução de $(2.61)$ para reescrever a função custo do problema de otimização. Integrando ambos os lados da equação (2.59):

$$
\begin{gathered}
\int_{0}^{\infty} \frac{d\left(\boldsymbol{x}^{\top} P \boldsymbol{x}\right)}{d t} d t=-\int_{0}^{\infty} \boldsymbol{x}^{\top}\left(Q+G^{\top} R G\right) \boldsymbol{x} d t \\
\lim _{t \rightarrow \infty} \boldsymbol{x}^{\top}(t) P \boldsymbol{x}(t)-\boldsymbol{x}^{\top}(0) P \boldsymbol{x}(0)=-J
\end{gathered}
$$


mas como a origem do sistema em malha fechada é um ponto de equilíbrio estável, o limite é nulo. Logo:

$$
J=\boldsymbol{x}^{\top}(0) P \boldsymbol{x}(0)
$$

Portanto, o problema de minimizar a função custo (2.56) sujeita à restrição (2.54) é equivalente a minimização de (2.64) sujeita à restrição (2.61). Como neste caso a restrição é dada por uma igualdade, pode-se utilizar o método dos multiplicadores de Lagrange para determinar as condições necessárias de minimização. Utilizando a função traço de uma matriz, é possível reescrever a função custo como:

$$
J=\operatorname{tr}\{P X\}+\operatorname{tr}\{S T\}
$$

em que $T$ representa a restrição dada pela equação (2.61), $X$ uma matriz dada pelo produto $\boldsymbol{x}(0) \boldsymbol{x}^{\top}(0)$ e $S$ uma matriz simétrica de multiplicadores de Lagrange. As condições necessárias para obtenção de um mínimo desta função custo são:

$$
\begin{array}{r}
\frac{\partial J}{\partial S}=A_{c}^{\top} P+P A_{c}+Q+G^{\top} R G=0 \\
\frac{\partial J}{\partial P}=A_{c} S+S A_{c}^{\top}+X=0 \\
\frac{1}{2} \frac{\partial J}{\partial G}=R G S-B^{\top} P S=0
\end{array}
$$

as quais podem ser colocadas somente em função da variável $P$. Resolvendo a equação (2.66c) para $G$, chega-se em:

$$
G=R^{-1} B^{\top} P
$$

que substituindo em (2.66a), têm-se:

$$
A^{\top} P+P A+Q-P B R^{-1} B^{\top} P=0
$$

A qual é conhecida como equação algébrica de Riccati (ARE). Resolvendo esta equação para a matriz $P$ e substituindo o resultado na equação (2.67), chega-se na lei de controle ótima. Cabe ressaltar que dadas as considerações realizadas no desenvolvimento anterior, a ARE possui solução única (KALMAN, 1960), garantindo que este problema de otimização é convexo. Além disso, não foi necessária a solução da equação (2.66b) para obtenção da lei de controle, mostrando que esta é independente da condição inicial do sistema.

A partir deste resultados é possível levantar alguns questionamentos, como quando uma lei de controle que estabiliza um sistema é ótima para uma determina função custo 
quadrática, ou quais as propriedades de um controlador ótimo em relação às margens de ganho e de fase. O primeiro caso é chamado de problema de controle ótimo inverso, e foi resolvido para sistemas lineares com entrada única em (KALMAN, 1964). É possível obter a solução deste problema a partir de uma manipução da ARE. Com este fim, substitui-se a matriz $Q$ pelo produto de uma matriz com sua transponsta $H^{\top} H$, a matriz $R$ pela unidade e utiliza-se a seguinte notação para o resolvente (KATO, 2013) da matriz A:

$$
\Phi(s)=\left(s I-A^{-1}\right)
$$

em que $s$ representa uma variável complexa. Fazendo as substituições das matrizes definidas anteriormente e somando e subtraindo $s P$ da equação (2.68):

$$
P(s I-A)+\left(-s I-A^{\top}\right)=H^{\top} H-P B B^{\top} P
$$

Multiplicando esta equação à esquerda por $B^{\top} \Phi^{\top}(-s)$ e à direita por $\Phi(s) B$ :

$$
B^{\top} \Phi^{\top}(-s) P B+B^{\top} P \Phi(s) B=B^{\top} \Phi^{\top}(-s)\left(H^{\top} H-P B B^{\top} P\right) \Phi(s) B
$$

Adicionando a unidade de ambos os lados e utilizando a equação (2.67) para eliminar a matriz $P$ :

$$
[1+G \Phi(-s) B][1+G \Phi(s) B]=1+B^{\top} \Phi^{\top}(-s) H^{\top} H \Phi(s) B
$$

Adotando $s=i \omega$ :

$$
|1+G \Phi(i \omega) B|^{2}=1+|H \Phi(i \omega) B|^{2}
$$

A qual implica na seguinte relação:

$$
|1+G \Phi(i \omega) B| \geq 1
$$

De fato, como demonstrado em (KALMAN, 1964), a equação anterior representa uma condição suficiente e necessária para que um dado ganho de controle $G$ seja ótimo para alguma função custo da forma (2.55). Além disto, esta relação é um resultado conhecido da teoria de controle clássico e implica que a sensibilidade do sistema em malha fechada à variação de parâmetros tanto da planta como do controlador é diminuida utilizando o ganho de controle $G$, o que indica que o controle ótimo possui propriedades de robustez. Ademais, a partir deste resultado é possível mostrar que um sistema com uma lei de controle ótima possui margem de ganho infinita e margem de fase de no mínimo $60^{\circ}$ (ANDERSON; MOORE, 1971). 


\section{Posicionamento de Sensores e Atuadores no Controle com Realimentação de Saída}

No capítulo anterior, constatou-se que para utilizar o controle LQR é necessário medir todos os estados do sistema. Como tentativa de solucionar esta dificuldade, propôsse o controle ótimo com realimentação da saída, mencionado na introdução deste trabalho, o qual visava minimizar a função custo do regulador quadrático com restrição de realimentação do sinal medido. Um problema identificado nesta abordagem dava-se pelo fato de não se considerar as questões envolvendo o posicionamento dos sensores e atuadores, o que pode exercer grande impacto no desempenho do sistema de controle. Neste capítulo, estuda-se a minimização da função custo quadrática considerando as posições dos sensores e atuadores como variáveis, além da restrição de realimentação somente do sinal medido. Determinam-se as condições necessárias para minimização da função custo quadrática e, a partir desta análise, apontam-se os principais problemas desta abordagem e propõem-se soluções para cada um dos problemas identificados. Após a análise dos principais problemas, sugere-se uma metodologia para o posicionamento de sensores e atuadores no controle de vibrações com realimentação de saída. Adicionalmente, chega-se a conclusão de que o controle colocalizado com realimentação negativa de velocidade é uma solução do problema do regulador linear quadrático, resultado que até o momento da redação deste texto não foi encontrado na literatura.

\subsection{Condições Necessárias para Minimização da Função Custo}

Para obter as condições necessárias para o ganho ótimo de saída e posicionamento ótimo de sensores e atuadores, o seguinte sistema que representa uma estrutura flexível é considerado:

$$
\begin{gathered}
\dot{\boldsymbol{x}}=A \boldsymbol{x}+B(\alpha) \boldsymbol{u} \\
\boldsymbol{y}=C(\xi) \boldsymbol{x} \\
\boldsymbol{u}=-K \boldsymbol{y}
\end{gathered}
$$

em que $\boldsymbol{x} \in \mathbb{R}^{2 n}$ é o vetor de estados, $\boldsymbol{u} \in \mathbb{R}^{a}$ é o vetor de entrada, $A \in \mathbb{R}^{2 n \times 2 n}$ é a matriz de estados, $B \in \mathbb{R}^{2 n \times a}$ é a matriz de entrada, $C \in \mathbb{R}^{s \times 2 n}$ é a matriz de saída, $K \in \mathbb{R}^{a \times s}$ é o ganho de controle, $\alpha \in \mathbb{R}^{a \times m}$ representa as posições dos atuadores, $\xi \in \mathbb{R}^{s \times m}$ as posições dos sensores, $m$ é a dimensão da estrutura (no caso de uma estrutura unidimensional $m=1$, bidimensional, $m=2$ ), a o número de atuadores e $s$ o número de sensores. A 
equação (3.1b) identifica os estados medidos do sistema, enquanto (3.1c) caracteriza a relação de realimentação de saída. O ganho de controle e as posições dos dispositivos de controles são variáveis a serem determinadas de modo a minimizar a seguinte função custo:

$$
J=\int \boldsymbol{x}^{\top} Q \boldsymbol{x}+\boldsymbol{u}^{\top} R \boldsymbol{u} d t
$$

que conforme visto na seção 2.3, considerando que (3.1) é estabilizável, é equivalente à seguinte função custo:

$$
J=\operatorname{tr}\{P X\}+\operatorname{tr}\left\{S\left(A_{c}^{\top} P+P A_{c}+Q+(K C)^{\top} R K C\right)\right\}
$$

sendo que no caso de realimentação da saída, $A_{c}=A-B K C$.

No problema do regulador linear quadrático, o objetivo era determinar o ganho que minimizava a função custo (3.3). No presente caso, as matrizes de saída $(C)$ e entrada $(B)$ também são consideradas variáveis de otimização. A forma destas matrizes depende de fatores relacionados aos sensores e atuadores, como a quantidade, o tipo, a forma e as posições. Neste trabalho, para determinar as condições necessárias de otimalidade da função custo, somente as posições são consideradas como variáveis, de modo que o tipo, a forma e a quantidade são considerados predeterminados. Além de especificar um escopo de análise, estas hipóteses são adotadas para que seja possível escrever estas matrizes como funções diferenciáveis de seus argumentos, de modo que se possa calcular o gradiente da função custo. Esta afirmação implica na exclusão de sistemas de parâmetros concentrados, pois nestes o conjunto possível de posições para os dispositivos de controle é discreto. Assumindo que as matrizes de entrada e saída são funções de no mínimo classe $C^{1}$ das posições dos atuadores e sensores, as condições necessárias para a minimização de (3.3) são:

$$
\begin{gathered}
\frac{\partial J}{\partial S}=A_{c}^{\top} P+P A_{c}+Q+C^{\top} K^{\top} R K C=0 \\
\frac{\partial J}{\partial P}=A_{c} S+S A_{c}^{\top}+X=0 \\
\frac{1}{2} \frac{\partial J}{\partial K}=R K C S C^{\top}-B^{\top} P S C^{\top}=0 \\
\frac{1}{2} \frac{\partial J}{\partial \xi_{i j}}=\operatorname{tr}\left\{\left(S C^{\top} K^{\top} R K-S P B K\right) \frac{\partial C}{\partial \xi_{i}}\right\}=0 \quad i=1, \ldots, s, \quad j=1, \ldots, m \\
-\frac{1}{2} \frac{\partial J}{\partial \alpha_{i j}}=\operatorname{tr}\left\{K C S P \frac{\partial B}{\partial \alpha_{i}}\right\}=0 \quad i=1, \ldots, a, \quad j=1, \ldots, m
\end{gathered}
$$

As equações (3.4a)-(3.4c) são as condições necessárias determinadas por Levine e Athans, enquanto as equações (3.4d) e (3.4e) dão as localizações ótimas dos sensores e 
atuadores. Diferentemente do caso da realimentação total de estados, não é possível manipular estas equações de modo a se obter uma equação em função de uma única variável. Além disso, as equações (3.4d) e (3.4e) também dependem da variável $S$, indicando que as posições dos sensores e atuadores e o ganho ótimo dependem da condição inicial do sistema. Para que exista uma solução para as equações (3.4) é necessário que exista uma configuração dos parâmetros de otimização de modo que o sistema seja estável. Uma análise da existência de parâmetros para que uma estrutura flexível seja estabilizável, assim como sugestões para lidar com a dependência dos valores ótimos com relação à condição inicial do sistema são propostos nas próximas seções.

\subsection{Estabilização de Estruturas Flexíveis}

Conforme visto na seção 3.1, a existência de uma solução das equações (3.4) está relacionada com a existência de parâmetros de otimização que estabilizem o sistema. Apesar de este ser um problema ainda aberto para um sistema linear qualquer (SADABADI; PEAUCELLE, 2016), é possível mostrar que para estruturas flexíveis que possuem uma determinada forma esta solução sempre existe. Para mostrar este resultado, considera-se o seguinte sistema na forma modal com amortecimento diagonalizável:

$$
\boldsymbol{x}=\left[\begin{array}{l}
\boldsymbol{\eta} \\
\dot{\boldsymbol{\eta}}
\end{array}\right] \quad A=\left[\begin{array}{cc}
0 & I \\
-\Lambda & -D
\end{array}\right] \quad B=\left[\begin{array}{ccc}
\mathbf{0} & \ldots & \mathbf{0} \\
\boldsymbol{\phi}\left(\boldsymbol{\alpha}_{1}\right) & \ldots & \boldsymbol{\phi}\left(\boldsymbol{\alpha}_{a}\right)
\end{array}\right]
$$

em que $\boldsymbol{\eta} \in \mathbb{R}^{n}$ é o vetor de coordenadas modais, $I \in \mathbb{R}^{n \times n}$ a matriz identidade, $\Lambda \in \mathbb{R}^{n \times n}$ representa a matriz diagonal dos autovalores ou frequências naturais ao quadrado, $D \in$ $\mathbb{R}^{n \times n}$ a matriz diagonal de amortecimento, $\phi \in \mathbb{R}^{n}$ o vetor de autofunções ou autofunções aproximadas no caso da utilização de um método de discretização e $\boldsymbol{\alpha}_{i}$ a posição do iésimo atuador. A forma considerada para a matriz $B$ indica que a atuação no sistema é realizada através de forças concentradas nas posições $\boldsymbol{\alpha}_{i}$. No caso da matriz de saída do sistema, consideram-se as seguintes possibilidades:

$$
\begin{aligned}
& C=\left[\begin{array}{ccc}
\phi\left(\boldsymbol{\xi}_{1}\right) & \ldots & \phi\left(\boldsymbol{\xi}_{s}\right) \\
\mathbf{0} & \ldots & \mathbf{0}
\end{array}\right]^{\top} \\
& C=\left[\begin{array}{ccc}
0 & \ldots & 0 \\
\phi\left(\boldsymbol{\xi}_{1}\right) & \ldots & \phi\left(\boldsymbol{\xi}_{s}\right)
\end{array}\right]^{\top}
\end{aligned}
$$

sendo (3.6a) no caso de medição de posição e (3.6b) no caso de medição de velocidade. O vetor $\boldsymbol{\xi}_{i}$ representa a posição do i-ésimo sensor, e esta forma da matriz $C$ indica que os sensores utilizados são pontuais. 
Para mostrar que este sistema é estabilizável utiliza-se uma função de Lyapunov da forma (2.58):

$$
V=\boldsymbol{x}^{\top}\left[\begin{array}{cc}
\Lambda & 0 \\
0 & I
\end{array}\right] \boldsymbol{x}
$$

a qual está associada a energia do sistema em coordenadas modais. Calculando a derivada no tempo de (3.7) chega-se na seguinte expressão:

$$
\dot{V}=-2\left(\dot{\boldsymbol{\eta}}^{\top} D \dot{\boldsymbol{\eta}}-\boldsymbol{x}^{\top} B \boldsymbol{u}\right)
$$

Como a matriz $D$ é positiva definida, utilizando a equação (3.8) é possível concluir que a origem é assintoticamente estável para uma entrada nula, o que ocorre devido à dissipação de energia através do amortecimento. No caso de um sistema sem amortecimento, ainda é possível garantir a estabilidade através da realimentação negativa de velocidade de pares de sensores e atuadores colocalizados (Figura 3). Neste caso, as posições dos sensores e atuadores são iguais $\left(\boldsymbol{\xi}_{i}=\boldsymbol{\alpha}_{i}\right)$, e utilizando as equações (3.1b), (3.1c) e a relação $C=B^{\top}$, obtém-se a seguinte expressão para $\dot{V}$ :

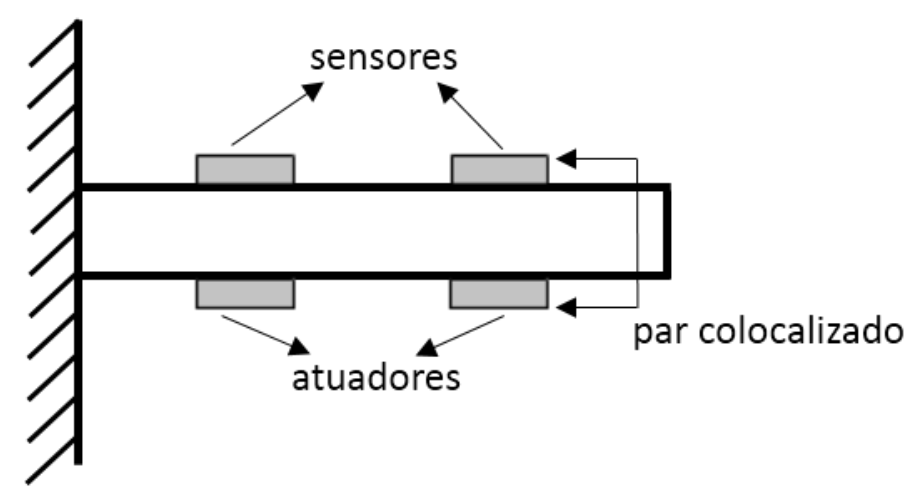

Figura 3 - Viga engastada com sensores e atuadores colocalizados.

$$
\dot{V}=-2 \boldsymbol{x}^{\top} B K B^{\top} \boldsymbol{x}
$$

mostrando que o sistema é estável para toda matriz $K$ positiva definida. No caso particular em que $K$ é uma matriz diagonal esta expressão pode ser escrita como:

$$
\dot{V}=-2 \sum_{i=1}^{a} k_{i}\left[\dot{\boldsymbol{\eta}}^{\top} \boldsymbol{\phi}\left(\boldsymbol{\alpha}_{i}\right)\right]^{2}
$$

sendo cada $k_{i}$ um termo da diagonal de $K$ que representa o ganho de controle do iésimo par colocalizado. Esta equação mostra que para valores positivos de $k_{i}$ o sistema é estável, e além disso, nota-se que a margem de ganho de cada um dos sensores é infinita, sugerindo que esta lei de controle pode ser ótima. De fato, pode-se mostrar que o controle 
colocalizado com realimentação negativa de velocidade é uma solução do problema do regulador linear quadrático. Com este objetivo, introduz-se a equação de Hamilton-JacobiBellman (HJB).

Dada a função custo:

$$
J=\int_{0}^{\infty} \boldsymbol{x}^{\top} Q \boldsymbol{x}+\boldsymbol{u}^{\top} R \boldsymbol{u} d t
$$

associada ao seguinte sistema dinâmico:

$$
\dot{\boldsymbol{x}}=\boldsymbol{f}(\boldsymbol{x})+G(\boldsymbol{x}) \boldsymbol{u}
$$

Pode-se demonstrar que o valor mínimo $J^{*}$ de (3.11) satisfaz a seguinte equação:

$$
\min _{\boldsymbol{u}}\left\{\boldsymbol{x}^{\top} Q \boldsymbol{x}+\boldsymbol{u}^{\top} R \boldsymbol{u}+\frac{\partial J^{*}}{\partial \boldsymbol{x}}[\boldsymbol{f}(\boldsymbol{x})+G(\boldsymbol{x}) \boldsymbol{u}]\right\}=0
$$

a qual representa a equação estacionária de HJB. A lei de controle $\boldsymbol{u}^{*}$ que minimiza esta equação é dada por:

$$
\boldsymbol{u}^{*}=-\frac{1}{2} R^{-1} G^{\top}\left(\frac{\partial J^{*}}{\partial \boldsymbol{x}}\right)^{\top}
$$

que substituindo em (3.13) implica na seguinte relação:

$$
\boldsymbol{x}^{\top} Q \boldsymbol{x}+\frac{\partial J^{*}}{\partial \boldsymbol{x}} \boldsymbol{f}(\boldsymbol{x})-\frac{1}{4} \frac{\partial J^{*}}{\partial \boldsymbol{x}} G(\boldsymbol{x}) R^{-1} G^{\top}(\boldsymbol{x})\left(\frac{\partial J^{*}}{\partial \boldsymbol{x}}\right)^{\top}=0
$$

Conforme demostrado em (FREEMAN; KOKOTOVIC, 2008), toda clf é uma solução da equação estacionária de HJB. Logo, a partir da clf $V$ dada em (3.7) é possível determinar uma lei de controle ótima para o sistema (3.5). Inicialmente, calculam-se os termos $\frac{\partial V}{\partial x} f$ e $\frac{\partial V}{\partial x} G$ :

$$
\begin{gathered}
\frac{\partial V}{\partial \boldsymbol{x}} f=\frac{\partial}{\partial \boldsymbol{x}}\left\{\boldsymbol{x}^{\top}\left[\begin{array}{ll}
\Lambda & 0 \\
0 & I
\end{array}\right] \boldsymbol{x}\right\}\left[\begin{array}{cc}
0 & I \\
-\Lambda & -D
\end{array}\right] \boldsymbol{x}=-2 \dot{\boldsymbol{\eta}}^{\top} D \dot{\boldsymbol{\eta}} \\
\frac{\partial V}{\partial \boldsymbol{x}} G=\frac{\partial}{\partial \boldsymbol{x}}\left\{\boldsymbol{x}^{\top}\left[\begin{array}{cc}
\Lambda & 0 \\
0 & I
\end{array}\right] \boldsymbol{x}\right\}\left[\begin{array}{ccc}
\mathbf{0} & \ldots & \mathbf{0} \\
\boldsymbol{\phi}\left(\boldsymbol{\alpha}_{1}\right) & \ldots & \boldsymbol{\phi}\left(\boldsymbol{\alpha}_{a}\right)
\end{array}\right]=2 \boldsymbol{x}^{\top} B
\end{gathered}
$$

Substituindo (3.16b) em (3.14) e adotando $R=I$ por simplicidade, encontra-se a seguinte lei de controle ótima:

$$
u_{i}=-\boldsymbol{\phi}^{\top}\left(\boldsymbol{\alpha}_{i}\right) \dot{\boldsymbol{\eta}}, \quad i=1, \ldots, a
$$


que representa exatamente a lei de controle obtida através da realimentação de velocidade de pares colocalizados. Para encontrar a função custo minimizada por esta lei de controle basta substituir as equações dadas em (3.16) na equação (3.15):

$$
\boldsymbol{x}^{\top} Q \boldsymbol{x}=2 \dot{\boldsymbol{\eta}}^{\top} D \dot{\boldsymbol{\eta}}+\boldsymbol{x}^{\top} B B^{\top} \boldsymbol{x}
$$

que implica em uma função custo do tipo (3.11) com $R=I$ e a seguinte matriz $Q$ :

$$
Q=\left[\begin{array}{cc}
0 & 0 \\
0 & 2 D+\sum_{i=1}^{a} \boldsymbol{\phi}\left(\boldsymbol{\alpha}_{i}\right) \boldsymbol{\phi}^{\top}\left(\boldsymbol{\alpha}_{i}\right)
\end{array}\right]
$$

Analisando a equação (3.18), nota-se que o segundo termo do lado direito da igualdade representa a soma dos quadrados das velocidades dos pontos em que estão os pares colocalizados. Portanto, para um sistema com amortecimento desprezível, o controle com realimentação negativa de velocidade minimiza uma função custo que representa uma medida da energia cinética dos pontos em que estão o pares colocalizados com o menor esforço. Além disso, por ser um controle ótimo, no caso de um sistema com um par colocalizado, esta lei de controle compreende todas as propriedades mencionadas na seção 2.3, como margem de ganho infinita, margem de fase de no mínimo $60^{\circ}$ e robustez.

\subsection{Condição Inicial do Sistema}

Um outro problema identificado na seção 3.1 é a dependência do ponto ótimo em relação à condição inicial do sistema. Conforme analisado na seção 1.1 deste trabalho, a maneira usual de lidar com esta dependência é assumir que a condição inicial é uma variável aleatória uniformemente distribuída em uma esfera de dimensão igual a do estado do sistema (LEVINE; ATHANS, 1970), que implica na substituição da matriz $X$ na equação (3.4b) pela matriz identidade. Um problema evidente desta abordagem é o fato de se dar a mesma importância para todos os estados do sistema. Apesar disso, na mesma seção verificou-se que não foram sugeridas propostas diferentes para lidar com este problema, além de também não se avaliar as possíveis diferenças ao se utilizar uma condição inicial distinta. Nesta seção, são propostas duas alternativas para lidar com esta dependência, as quais serão avaliadas e comparadas com a proposta de (LEVINE; ATHANS, 1970) no Capítulo 4 através de exemplos numéricos. A primeira delas consiste em aproximar o desempenho do controle com realimentação de saída do controle com realimentação de estados para qualquer condição inicial. O problema desta proposta é que, para comparar o desempenho do controle com realimentação de saída com o LQR, as posições dos atuadores devem ser predeterminadas. Para lidar com esta dificuldade, sugere-se uma segunda proposta em que se especifica um conjunto de possíveis condições iniciais para o sistema e otimiza-se para a condição que implica no maior valor para a função custo. As 
duas popostas sugeridas possuem uma abordagem de otimização robusta, que consiste em otimizar para o pior caso.

\subsubsection{Aproximação entre os Controles com Realimentação de Saída e de Es- tados}

De acordo com o que foi visto na seção 2.3, o ganho de controle obtido através da solução do regulador linear quadrático é independente da condição inicial do sistema, o que implica que a função custo associada a esse ganho assume um valor mínimo para qualquer condição inicial. Logo, um modo de avaliar o desempenho do controle com realimentação de saída é comparar o valor de sua função custo com a do LQR para diferentes condições iniciais. A sugestão proposta nesta subseção para lidar com a dependência da condição inicial é baseada nesta comparação, e consiste em otimizar a razão entre as funções custo do controle com realimentação de saída e do LQR para a condição inicial em que esta razão assume o maior valor. Para fazer esta comparação, é necessário determinar o ganho para o LQR, o que implica que as posições dos atuadores devem ser predeterminadas. Logo, as variáveis do problema são a posição dos sensores $(\xi)$ e o ganho de controle $(K)$, e a otimização pode ser escrita da seguinte forma:

$$
\min _{(\xi, K)} \max _{\boldsymbol{x}_{0}} \frac{\boldsymbol{x}_{0}^{\top} P_{s}(\xi, K) \boldsymbol{x}_{0}}{\boldsymbol{x}_{0}^{\top} P_{l} \boldsymbol{x}_{0}}
$$

em que os subscritos $s$ e $l$ referem-se ao controle de saída e LQR, respectivamente. A forma (2.64) foi utilizada para a função custo.

Este problema possui uma dificuldade potencialmente maior, pois a princípio é necessária a solução de dois problemas de otimização. No entanto, para o problema de encontrar a condição inicial que maximiza a razão entre os critérios de desemepenho, determinou-se uma solução relativamente simples. Como $P_{s}$ e $P_{l}$ são positivas definidas e simétricas, é possível realizar uma diagonalização simultânea de ambas, e a matriz que as diagonaliza é formada pelos autovetores que são solução do problema de autovalor generalizado (LAUB, 2005):

$$
P_{s} z=\lambda P_{l} \boldsymbol{z}
$$

Definindo a base de autovetores como $\left\{\boldsymbol{z}_{1}, \ldots, \boldsymbol{z}_{2 n}\right\}$, é possível escrever a condição inicial do sistema como:

$$
\boldsymbol{x}_{0}=\sum_{i=1}^{2 n} c_{i} \boldsymbol{z}_{i}
$$


Além disso, como essa base diagonaliza ambas as matrizes é possível mostrar que:

$$
\boldsymbol{z}_{r}^{\top} P_{s} \boldsymbol{z}_{t}=0, \quad \boldsymbol{z}_{r}^{\top} P_{l} \boldsymbol{z}_{t}=0, \quad r, t=1, \ldots, 2 n \quad r \neq t
$$

Substituindo (3.22) na razão dada em (3.20) e utilizando as relações de ortogonalidade (3.23), chega-se na seguinte expressão:

$$
\frac{\sum_{i=1}^{2 n} c_{i}^{2} \boldsymbol{z}_{i}^{\top} P_{s} \boldsymbol{z}_{i}}{\sum_{i=1}^{2 n} c_{i}^{2} \boldsymbol{z}_{i}^{\top} P_{l} \boldsymbol{z}_{i}}
$$

É possível mostrar que o valor máximo dessa expressão é dado pelo maior autovalor do problema generalizado (3.21), e a condição inicial que a maximiza é dada pelo autovetor correspondente. Primeiro, calcula-se o valor das funções custo associadas a cada um dos autovetores:

$$
J_{s i}=\boldsymbol{z}_{i}^{\top} P_{s} \boldsymbol{z}_{i}, \quad J_{l i}=\boldsymbol{z}_{i}^{\top} P_{l} \boldsymbol{z}_{i}, \quad i=1, \ldots, 2 n
$$

então, calcula-se a razão $J_{s i} / J_{l i}$ para cada um desses autovetores. Assumindo que esta razão assume o maior valor para o autovetor $\boldsymbol{z}_{r}$, têm-se que:

$$
\frac{J_{s r}}{J_{l r}} \geq \frac{J_{s i}}{J_{l i}} \Longleftrightarrow J_{s r} J_{l i}-J_{l r} J_{s i} \geq 0, \quad i=1, \ldots, 2 n
$$

Multiplicando o lado direito desta expressão por $c_{i}^{2}$ para cada valor de $i$ e somando para todos os valores desse índice:

$$
\begin{gathered}
\sum_{i=1}^{2 n} c_{i}^{2}\left(J_{s r} J_{l i}-J_{l r} J_{s i}\right) \geq 0 \\
\frac{J_{s r}}{J_{l r}} \geq \frac{\sum_{i=1}^{2 n} c_{i}^{2} J_{s i}}{\sum_{i=1}^{2 n} c_{i}^{2} J_{l i}} \\
\frac{\boldsymbol{z}_{r}^{\top} P_{s} \boldsymbol{z}_{r}}{\boldsymbol{z}_{r}^{\top} P_{l} \boldsymbol{z}_{r}} \geq \frac{\sum_{i=1}^{2 n} c_{i}^{2} \boldsymbol{z}_{i}^{\top} P_{s} \boldsymbol{z}_{i}}{\sum_{i=1}^{2 n} c_{i}^{2} \boldsymbol{z}_{i}^{\top} P_{l} \boldsymbol{z}_{i}}
\end{gathered}
$$


provando que a razão assume valor máximo para o autovetor $\boldsymbol{z}_{r}$. Substituindo este autovetor em (3.21), multiplicando à esquerda por $\boldsymbol{z}_{r}^{\top}$ e isolando o autovalor chega-se em:

$$
\lambda_{r}=\frac{\boldsymbol{z}_{r}^{\top} P_{s} \boldsymbol{z}_{r}}{\boldsymbol{z}_{r}^{\top} P_{s} \boldsymbol{z}_{r}}
$$

mostrando que o maior valor da razão é dado pelo maior autovalor. Portanto, para determinar a condição inicial que maximiza (3.24) e o maior valor desta expressão, basta resolver o problema generalizado de autovalor (3.21) e determinar o maior autovalor e o autovetor correspondente. Esta abordagem possui a vantagem de não ser necessário especificar nada sobre a condição inicial do sistema.

\subsubsection{Conjunto de Possíveis Condições Iniciais}

A abordagem proposta na seção anterior possui a desvantagem de não ser aplicável para a determinação das posições dos atuadores, pois ela é baseada em comparar o desempenho do controle de saída com o LQR. A proposta desta subseção consiste em especificar um possível conjunto de condições iniciais para o sistema e otimizar para a condição que implica no maior valor para a função custo. Esta abordagem possui a vantagem de também ser aplicável para a determinação das posições dos atuadores no controle LQR, de modo que é possível utilizá-la de forma complementar a abordagem anterior.

O desenvolvimento a seguir é baseado em (BEN-TAL; GHAOUI; NEMIROVSKI, 2009). Inicialmente, define-se o seguinte conjunto para parametrizar as possíveis condições iniciais do sistema:

$$
\chi=\left\{\boldsymbol{\zeta} \in \mathbb{R}^{2 n} \mid\|\boldsymbol{\zeta}\|_{\infty} \leq 1\right\}
$$

E usando este conjunto, afirma-se que as condições iniciais mais prováveis de ocorrerem no sistema real pertencem ao seguinte conjunto:

$$
\Omega=\left\{\boldsymbol{x}_{0} \in \mathbb{R}^{2 n} \mid \boldsymbol{x}_{0}=\boldsymbol{z}_{0}+\sum_{i=1}^{2 n} \zeta_{i} \boldsymbol{\delta}_{i}\right\}
$$

em que $\boldsymbol{z}_{0}$ é um valor central, $\zeta_{i}$ é o i-ésimo componente do vetor $\boldsymbol{\zeta}$ e $\boldsymbol{\delta}_{i}$ é um vetor cujo i-ésimo componente é uma constante positiva $\delta_{i}$ e todas as outras são nulas. A variável $\delta_{i}$ representa o máximo valor esperado para a variável de estado $x_{i}$ do sistema. Em outras palavras, o conjunto das condições iniciais mais prováveis de ocorrem é uma caixa de dimensão $2 n$ centrada em $\boldsymbol{z}_{0}$, em que cada aresta mede $2 \delta_{i}$. Definindo a variável:

$$
\boldsymbol{\Delta}=\sum_{i=1}^{2 n} \boldsymbol{\delta}_{i}
$$


e substituindo $\boldsymbol{x}_{0}$ dado na equação (3.30) em (3.3), determina-se o maior valor que a função custo assume para uma condição inicial que pertence ao conjunto (3.30):

$$
\bar{J}=\operatorname{tr}\left\{P\left(\boldsymbol{z}_{0} \boldsymbol{z}_{0}^{\top}+\boldsymbol{z}_{0} \boldsymbol{\Delta}^{\top}+\boldsymbol{\Delta} \boldsymbol{z}_{0}^{\top}+\boldsymbol{\Delta} \boldsymbol{\Delta}^{\top}\right)\right\}
$$

em que foi utilizado o fato de que para uma configuração de parâmetros de otimização em que o sistema é estável, o segundo termo do lado direito da igualdade em (3.3) é nulo.

Assim como na proposta de (LEVINE; ATHANS, 1970), nesta abordagem também se especifica um conjunto para as condições iniciais, com a diferença que para esta não é necessário identificar a distribuição. Além disso, esta abordagem proporciona ao projetista a liberdade de especificar um conjunto de possíveis condições iniciais que possam fazer sentido para o sistema em análise, apesar de que esta pode não ser uma tarefa simples para qualquer caso. Assim como na seção anterior, também seria possível propor uma abordagem em que não fosse necessário especificar um conjunto para as condições iniciais, utilizando por exemplo a decomposição em valores singulares da matriz P. Assim, seria possível determinar a condição inicial que implica em um maior valor absoluto da função custo e otimizar para este caso. No entanto, verificou-se numericamente que esta proposta gerava resultados piores do que a abordagem proposta nesta subseção.

\subsection{Metodologia}

A partir das discussões realizadas nas seções anteriores, propõe-se uma metodologia para o posicionamento de sensores e atuadores no controle de vibrações com realimentação de saída. Conforme sugerido na seção 3.3, a metodologia é baseada em primeiro determinar posições dos atuadores para obter um controle LQR com desempenho satisfatório e, em seguida, determinar as posições dos sensores e ganho de controle para conseguir um desempenho semelhante ao obtido pelo LQR para qualquer configuração do sistema.

Conforme visto na seção 2.3, o ganho de controle para o LQR não depende da condição inicial do sistema. No entanto, de acordo com a equação (3.4e), a qual também é valida para o LQR (substituindo $K C$ por $G$ ), as posições ótimas dos atuadores também dependem da condição inicial. Logo, consoante a abordagem proposta na subseção 3.3.2, o primeiro passo neste caso é determinar um conjunto de possíveis condições iniciais. Após esta determinação basta aplicar um método numérico para resolver as equações (3.4a), (3.4b) (sendo $X$ a matriz que multiplica $P$ em (3.32)), (3.4c) e (3.4e), ou aplicar um método para otimizar diretamente a função custo (3.32). Neste trabalho, optou-se pela segunda alternativa. Por esta otimização ser não convexa, devem ser utilizadas diferentes valores iniciais no algoritmo. Diferentemente do caso de realimentação de saída, a estabilização na realimentação de estados não é um problema pois para quaisquer posições dos atuadores o sistema é estabilizável. 
Entre os diversos métodos numéricos que podem ser utilizados, como os apresentados em (NOCEDAL; WRIGHT, 2008), optou-se pela utilização do método da programação sequencial quadrática (SQP), devido a sua eficiência para resolver problemas com restrições (HOCK; SCHITTKOWSKI, 1983) e sua disponibilidade em softwares comerciais como o MATLAB. O método SQP pode ser formulado tanto como um método de busca em linha ou como um método de região de confiança. No caso do método de busca em linha, a matriz Hessiana do problema deve ser positiva definida (NOCEDAL; WRIGHT, 2008), sendo que nos casos em que isso não ocorre utiliza-se uma aproximação que seja positiva definida ou aplicam-se modificações na matriz durante o processo de fatorização. Para o caso de região de confiança esta imposição não é necessária, no entanto, devido a inclusão de restrições para este caso o custo computacional é maior e em alguns casos a otimização pode se tornar infactível. Conforme a documentação do MATLAB (MATLAB, 2015), no algoritmo empregado formula-se o SQP como um método de busca em linha, em que a Hessiana é estimada assim como no método BFGS.

Após a determinação das posições dos atuadores e do controle LQR, utiliza-se a proposta dada na subseção 3.3.1 para o posicionamento dos sensores e determinação do ganho de realimentação de saída. Neste caso não é necessário especificar nada sobre a condição inicial do sistema, porém, há um problema em relação ao valor inicial do algoritmo. De acordo com o que foi visto na seção 3.2, estruturas flexíveis são sempre estabilizáveis, e poderia ser utilizada a configuração colocalizada como chute inicial do algoritmo. No entanto, o problema de otimização neste caso é não convexo, e é pertinente a avaliação da solução a partir da utilização de diferentes valores iniciais.

A primeira alternativa encontrada para lidar com este problema, foi a proposta de um novo problema de otimização. A partir da aplicação do controle LQR para diferentes estruturas, observou-se que o desempenho do sistema de controle praticamente não se modificava quando os ganhos relacionados à realimentação de deslocamento eram anulados. Baseado nesta observação, propôs-se a seguinte otimização:

$$
\min _{\xi}\|G-K C(\xi)\|_{2}
$$

a qual consiste em aproximar o produto entre o ganho e a matriz de saída com realimentação de velocidade, do ganho ótimo obtido pelo LQR em que os termos relacionados a realimentação de deslocamento são nulos. $K$ pode não ser considerada uma variável de otimização neste caso pois, para dadas posições $\xi, K$ pode ser determinada através do método dos mínimos quadrados. Esta otimização é não convexa, e pode gerar diferentes valores iniciais para serem utilizados para o problema de otimização (3.20). A aplicação desta otimização para o caso de um viga engastada gerou bons resultados, e para todas as posições aleatórias utilizadas como valores iniciais em (3.33), sempre foi possível encontrar um par $(\xi, K)$ que estabilizasse o sistema. No entanto, para uma placa engastada 
o resultado obtido foi diferente, e para alguns valores iniciais utilizados as posições dos sensores convergiam para pontos próximos do engaste, de modo que os ganhos tendiam a infinito. Portanto, optou-se por gerar posições e ganhos aleatórios até encontrar uma configuração em que o sistema fosse estável. Em ambos os casos testados no Capítulo 4, verificou-se que independentemente das posições dos atuadores sempre foi possível encontrar diferentes combinações $(\xi, K)$ que deixavam o sistema estável.

Após obter um par $(\xi, K)$ em que o sistema é estável, este é utilizado como valor inicial para a otimização (3.20). Para o problema de maximização utilizou-se o procedimento proposto na subseção 3.3.1, enquanto para a minimização também foi utilizado o algoritmo SQP. Para cada iteração deve ser determinada uma nova condição inicial que maximiza a razão entre os desempenhos, já que a matriz $P_{s}$ se altera de acordo com as posições e ganhos de controle calculados em cada iteração. Para o critério de convergência, considerou-se que a norma infinita do gradiente da função custo deveria ser menor que $1 \times 10^{-8}$. 


\section{Aplicações no Controle de Vibração de Es- truturas Flexíveis}

Neste capítulo avalia-se a metodologia proposta para o posicionamento de sensores e atuadores e determinação do ganho de realimentação de saída através de exemplos elementares de estruturas flexíveis. Também é realizada uma comparação entre a proposta dada em (LEVINE; ATHANS, 1970) e as abordagens desenvolvidas na seção 3.3 para lidar com a dependência em relação à condição do sistema. O desempenho dos controladores obtidos são comparados com o LQR, considerando como métricas algumas características da resposta no tempo, a magnitude da função custo e a resposta em frequência.

\subsection{Viga Engastada-Livre}

O primeiro exemplo a ser considerado é o de uma viga engastada-livre com sensores e atuadores pontuais. A Figura 4 ilustra um sistema em que se pode considerar que o atuador e os sensores são pontuais.

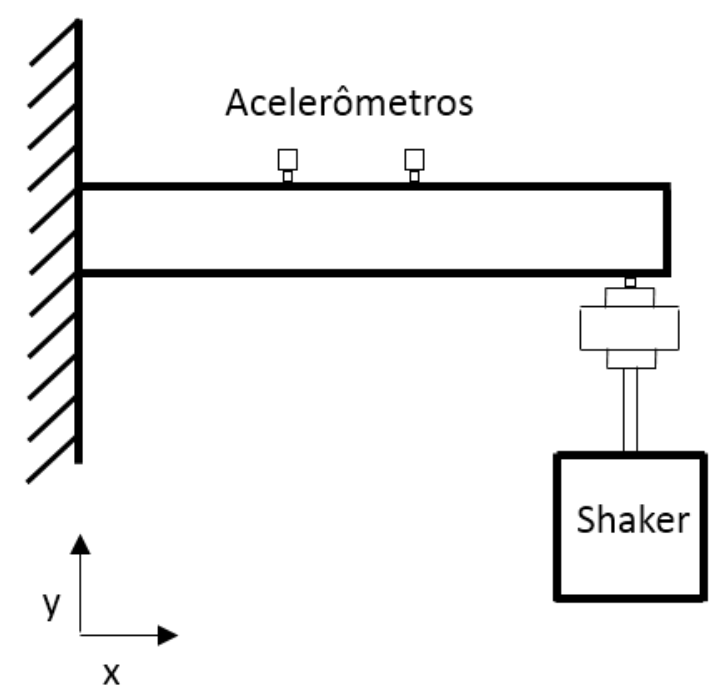

Figura 4 - Viga engastada com atuador e sensores pontuais.

\subsubsection{Modelo e Projeto do Sistema de Controle}

O modelo considerado pode ser obtido através da teoria de Euler-Bernoulli, a qual considera as seguintes hipóteses (CRAIG; KURDILA, 2006): 
- O plano $x y$ é denominado plano principal da viga e qualquer seção transversal pertencente a esse plano permanece plana durante o movimento transversal da viga;

- Existe um eixo longitudinal da viga que não sofre deformação ou extensão, o qual é chamado de eixo neutro;

- As seções perpendiculares ao eixo neutro permanecem planas, o que implica que a deformação por cisalhamento é desconsiderada;

- O material é linearmente elástico, com módulo de elasticidade $E(x)$;

- As tensões $\sigma_{y}$ e $\sigma_{z}$ são desprezíveis quando comparadas com a tensão $\sigma_{x}$;

- A inércia de rotação é desconsiderada;

- A densidade $\rho(x)$ é constante ao longo da seção transversal.

Considerando estas hipóteses e aplicando o princípio estendido de Hamilton ou as equações de Newton, é possível obter um modelo para a viga em flexão conforme as equações (2.1), com $L$ dado por:

$$
L=\frac{\partial^{2}}{\partial x^{2}}\left(E I \frac{\partial^{2}}{\partial x^{2}}\right)
$$

em que $I$ representa o segundo momento de área da seção transversal. No caso da viga engastada-livre, a deflexão e a inclinação da viga no ponto de engaste devem ser nulos. Na extremidade livre, tanto o momento de flexão quanto a força de cisalhamento também devem ser nulos, de modo que as condições de contorno podem ser escritas na forma (2.6):

$$
\begin{gathered}
B_{1}=1, \quad B_{2}=\frac{\partial}{\partial x}, \quad x=0 \\
B_{1}=\frac{\partial}{\partial x}\left(E I \frac{\partial^{2}}{\partial x^{2}}\right), \quad B_{2}=E I \frac{\partial^{2}}{\partial x^{2}}, \quad x=L
\end{gathered}
$$

Para o caso em que a densidade, o módulo de elasticidade e o momento de inércia de área são constantes, é possível obter uma solução analítica para o problema diferencial de autovalor. Apesar de no exemplo considerado estas características serem assumidas, de modo que seria possível utilizar a solução analítica, optou-se por utilizar o método de elementos finitos. Para garantir uma boa aproximação tanto para as frequências naturais quanto para as autofunções, dividiu-se a viga em 50 elementos. Como funções de interpolação foram utilizadas as cúbicas de Hermite (2.51). Para determinar numericamente as matrizes de massa e rigidez (2.36), são necessários os parâmetros da viga. No presente caso, escolheu-se estudar uma viga de aço de seção retangular, cujos parâmetros são dados na Tabela 1. 
Tabela 1 - Características da viga estudada.

\begin{tabular}{lc}
\hline \hline Densidade $\left(\mathrm{kg} / \mathrm{m}^{3}\right)$ & 7860 \\
Módulo de Elasticidade $(\mathrm{GPa})$ & 200 \\
x-Comprimento $(\mathrm{mm})$ & 300 \\
z-Largura $(\mathrm{mm})$ & 30 \\
y-Altura $(\mathrm{mm})$ & 3 \\
\hline \hline
\end{tabular}

A partir destas propriedades foram calculadas as matrizes de rigidez e massa, em que os termos associados ao deslocamento e inclinação do ponto de engaste foram anulados. Após a determinação destas matrizes, solucionou-se o problema algébrico de autovalor (2.35), e determinaram-se as frequências naturais e as autofunções através da equação (2.37). Para o método de elementos finitos, $\boldsymbol{\psi}$ é um vetor em que cada elemento é dado pela soma de todas as funções que multiplicam a coordenada correspondente. O modelo foi truncado até o $10^{\circ}$ modo, de modo que o sistema em espaço de estados fosse constituído por 20 estados. As frequências naturais do modelo foram comparadas com as frequências teóricas, as quais são dadas pela seguinte fórmula:

$$
\omega_{i}=\beta_{i}^{2} \sqrt{\frac{E I}{\rho A L^{4}}}
$$

em que $A$ é a área da seção transversal e $\beta_{i}$ são as raízes positivas da equação:

$$
\cos \beta_{i} \cosh \beta_{i}=-1
$$

As frequências naturais do modelo e teóricas estão indicadas na tabela a seguir, expressas em Hz:

Tabela 2 - Comparação entre os valores de frequência teóricos e os obtidos pelo modelo em elementos finitos.

\begin{tabular}{cccc}
\hline \hline Modo & Frequência Teórica & Frequência Modelo & Erro(\%) \\
\hline $1^{\circ}$ & 27,16 & 27,16 & $1 \times 10^{-6}$ \\
$2^{\circ}$ & 170,22 & 170,22 & $5 \times 10^{-6}$ \\
$3^{\circ}$ & 476,63 & 476,63 & $4.2 \times 10^{-5}$ \\
$4^{\circ}$ & 934,00 & 934,00 & $1.62 \times 10^{-4}$ \\
$5^{\circ}$ & 1543,96 & 1543,97 & $4.42 \times 10^{-4}$ \\
$6^{\circ}$ & 2306,41 & 2306,44 & $9.85 \times 10^{-4}$ \\
$7^{\circ}$ & 3221,35 & 3221,42 & $1.919 \times 10^{-3}$ \\
$8^{\circ}$ & 4288,78 & 4288,93 & $3.395 \times 10^{-3}$ \\
$9^{\circ}$ & 5508,71 & 5509,01 & $5.589 \times 10^{-3}$ \\
$10^{\circ}$ & 6881,12 & 6881,72 & $8.701 \times 10^{-3}$ \\
\hline \hline
\end{tabular}

A faixa de frequências estudada pode ser considerada larga para aplicações práticas, no entanto, um dos objetivos de trabalhar com um número maior de modos é avaliar o 
desempenho do controle para uma razão pequena entre número de sensores pelo número de estados. Ademais, conforme manual consultado (OMEGA, 2017), há acelerômetros disponíveis para medição nesta faixa de frequências. Além destas propriedades, adotou-se um fator de amortecimento $(\zeta)$ de $0,5 \%$ para cada um dos modos do sistema. A fim de obter o modelo completo do sistema ainda é necessário determinar as características do sistema de controle. Como um dos objetivos é comparar a resposta do controlador com realimentação da saída com o LQR, a fim de simplificar o sistema, optou-se por utilizar somente um atuador com uma entrada de força. Para realizar esta comparação, inicialmente é necessário projetar o controle LQR, que nesse caso consiste basicamente em determinar as matrizes de ponderação da função custo e especificar a posição do atuador. Seguindo os passos indicados na seção 3.4, inicialmente é necessário especificar um conjunto de condições iniciais mais prováveis para o sistema.

A ideia utilizada para determinar este conjunto é baseada em uma excitação estática, e consiste inicialmente em determinar qual ponto da estrutura atingirá o maior deslocamento dada a aplicação de uma força concentrada de uma dada magnitude, cuja posição pode ser modificada. Após a determinação da posição da força que proporciona o maior deslocamento, especifica-se um deslocamento máximo para este ponto e calcula-se o valor da força necessário para atingir esta configuração. Por fim, aplica-se esta força em cada um dos pontos da viga, e calcula-se o máximo deslocamento modal para cada um dos modos. O conjunto de possíveis condições iniciais para o sistema é da forma (3.30), em que cada $\delta_{i}$ é dado pelo máximo deslocamento modal correspondente. Para $\boldsymbol{z}_{0}$ assume-se o valor nulo, para incluir o caso de uma excitação simétrica.

No caso da viga engastada-livre este procedimento pode ser realizado sem dificuldades. Utilizando a equação (2.24), têm-se que o deslocamento modal correspondente a aplicação de uma força constante $F$ na posição $\boldsymbol{x}_{p}$ é dado por:

$$
\eta_{r}=\frac{F \phi_{r}\left(\boldsymbol{x}_{p}\right)}{\omega_{r}^{2}}, \quad r=1, \ldots, n
$$

sendo que neste caso $n$ é o número de modos em que a viga foi discretizada e $\phi_{r}$ é a r-ésima autofunção aproximada. Dadas estas considerações e substituindo (4.5) em (2.17):

$$
w\left(x_{p}\right)=F \sum_{r=1}^{n}\left(\frac{\phi_{r}\left(\boldsymbol{x}_{p}\right)}{\omega_{r}}\right)^{2}
$$

Logo, o ponto que possui maior deslocamento devido a aplicação de uma força concentrada constante é o valor de $\boldsymbol{x}_{p}$ que maximiza (4.6). No caso de uma viga engastada-livre, $\boldsymbol{x}_{p}$ é um escalar e o ponto em que (4.6) assume valor máximo é $x_{p}=L$, já que todas as autofunções assumem valor máximo em módulo na extremidade da viga, conforme a Figura 5. Na imagem foram indicados somente os cinco primeiros modos para facilitar a visualização. 


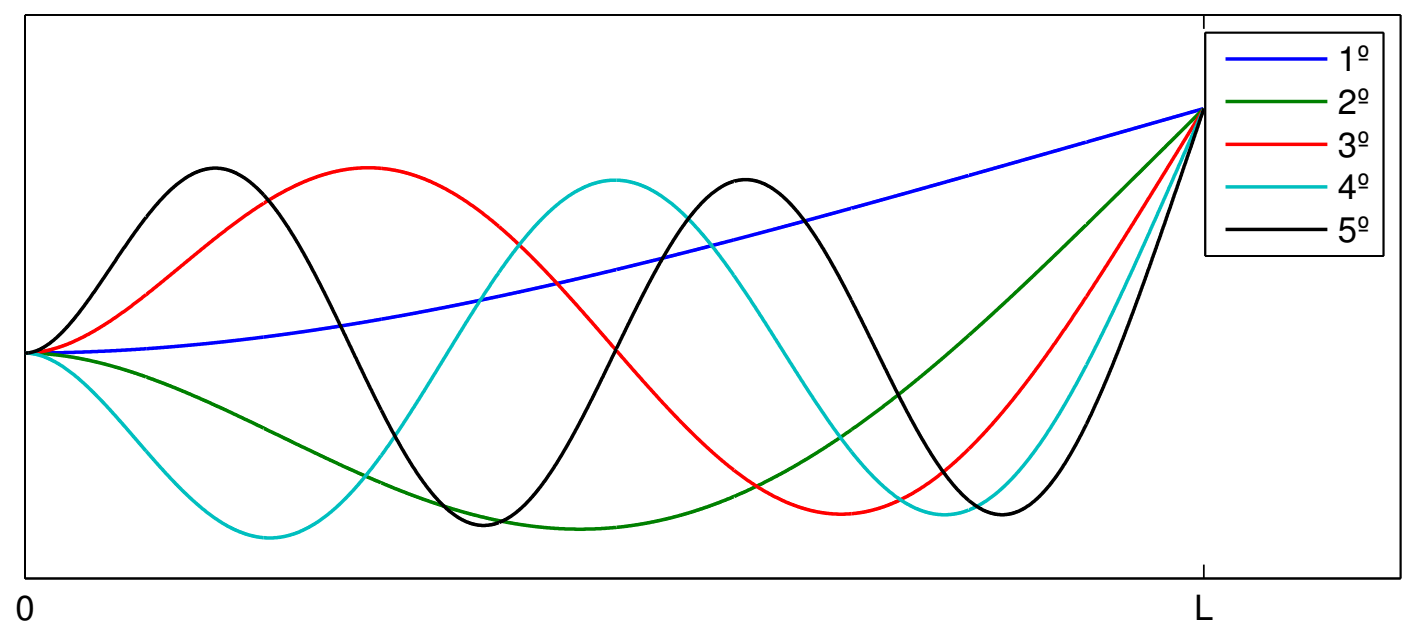

Figura 5 - Forma dos modos de vibrar de uma viga engastada-livre.

O próximo passo é determinar o máximo deslocamento admitido para a extremidade livre da viga. Como o modelo utilizado é baseado em pequenas deformações, escolheu-se um deslocamento máximo de $3 \mathrm{~mm}$, o qual é equivalente à altura da seção transversal da viga. Utilizando este valor e a equação (4.6), obteve-se a força necessária, de magnitude $4.5 \mathrm{~N}$. Aplicando esta força sobre cada um dos pontos da viga e calculando o valor do maior deslocamento correspondente, o que neste caso é equivalente a substituir a força $F$ obtida na equação (4.5), encontram-se os seguintes valores para os deslocamentos modais:

$$
\boldsymbol{\eta}_{M}=\left[\begin{array}{llllllllll}
67078 & 1708 & 218 & 57 & 21 & 9 & 5 & 3 & 2 & 1
\end{array}\right]^{\top} \times 10^{-8}
$$

Associando cada um destes valores às respectivas constantes $\delta_{i}$ e assumindo as constantes associadas às velocidades modais nulas, têm-se que:

$$
\boldsymbol{\Delta}=\left[\begin{array}{c}
\boldsymbol{\eta}_{M} \\
\mathbf{0}
\end{array}\right]
$$

Esta forma para o conjunto de possíveis condições iniciais prioriza o controle do primeiro modo, possuindo uma predileção um pouco menor para os modos de baixa frequência e quase nenhuma para os modos de alta frequência.

Para determinar completamente a função custo, ainda é necessário especificar as matrizes de ponderação de desempenho e esforço. Em relação à matriz de ponderação $Q$, foram realizadas algumas simulações comparando a matriz identidade com as sugestões encontradas em (MEIROVITCH; BARUH; OZ, 1983) e (HANAGUD; OBAL; CALISE, 1992) que são dadas respectivamente por uma ponderação dos deslocamentos modais pelos autovalores e pelo inverso dos autovalores. Apesar da pequena diferença entre os resultados 
obtidos, notou-se que o desempenho no caso da ponderação dada pelos autovalores era ligeiramente superior. Logo, adotou-se uma matriz $Q$ dessa forma:

$$
Q=\left[\begin{array}{ll}
\Lambda & 0 \\
0 & I
\end{array}\right]
$$

a qual, assim como a matriz $P$ utilizada em (3.7), está diretamente ligada a energia do sistema na representação em coordenadas modais. No caso da matriz de ponderação $R$, em pesquisas teóricas normalmente são arbitrados valores. Para um problema prático, esta matriz deve ser ajustada de acordo com a capacidade dos atuadores e potência disponível. A partir da consulta de um manual de um shaker eletrodinâmico (MODAL SHOP, 2009), verificou-se que mesmo shakers de baixa potência possuem limite de força de até $20 \mathrm{~N}$, valor bem acima do necessário para a viga atingir o deslocamento de $3 \mathrm{~mm}$ a partir de uma força constante. No caso da viga escolhida, verificou-se que, após a realização de simulações utilizando diferentes tipos de excitações, o valor $R=0.005$ proporcionava uma boa relação de compromisso entre desempenho e esforço empregado.

A partir destas considerações, é possível determinar a posição do atuador para o controle LQR. Para resolver a ARE e determinar tanto a matriz $P$ necessária para a função custo quanto o ganho de controle, utilizou-se a função lqr disponível no software MATLAB, a qual é baseada no artigo (ARNOLD; LAUB, 1984). Foram comparadas tanto a proposta de Levine e Athans quanto a proposta da subseção 3.3.2 para lidar com a dependência da posição do atuador em relação à condição inicial. Em ambos os casos obteve-se que a posição ótima do atuador era na extremidade livre da viga. Este resultado era esperado, já que, como se pode ver na Figura 5, esta posição é a melhor para o controle de todos os modos. Não obstante, considerou-se esta exposição neste instante da dissertação para exemplificar uma forma de determinar o conjunto de condições iniciais, já que a mesma é utilizada na seção 4.2 para determinação das posições dos atuadores no controle de vibração de uma placa.

Para realizar o cálculo das posições dos sensores e do ganho de realimentação de saída, é necessário determinar primeiro o tipo dos sensores que serão utilizados. Conforme já antecipado na seção 3.4, verificou-se que o desempenho do controle LQR praticamente não se altera quando os ganhos relacionados à realimentação de deslocamento são anulados. Utilizando o procedimento proposto na subseção 3.3.1, verificou-se que no pior caso, ou seja, para a condição inicial que maximiza a razão entre a função custo do sistema com somente realimentação de velocidade e a do sistema com realimentação de estados, a diferença relativa entre as funções custo é de somente $0.92 \%$. Este resultado também pode ser verificado na Figura 6 e na Figura 7, em que é possível notar que as curvas de ambos controladores são praticamente indistinguíveis. O termo LQRv foi utilizado para identificar o controle com realimentação somente de velocidade e a abreviação Mal. Ab. para o 
sistema sem controle. A partir deste resultado, optou-se por utilizar somente sensores de velocidade. Em todas as figuras da viga engastada-livre foi considerada uma medida de deslocamento do ponto na extremidade livre.

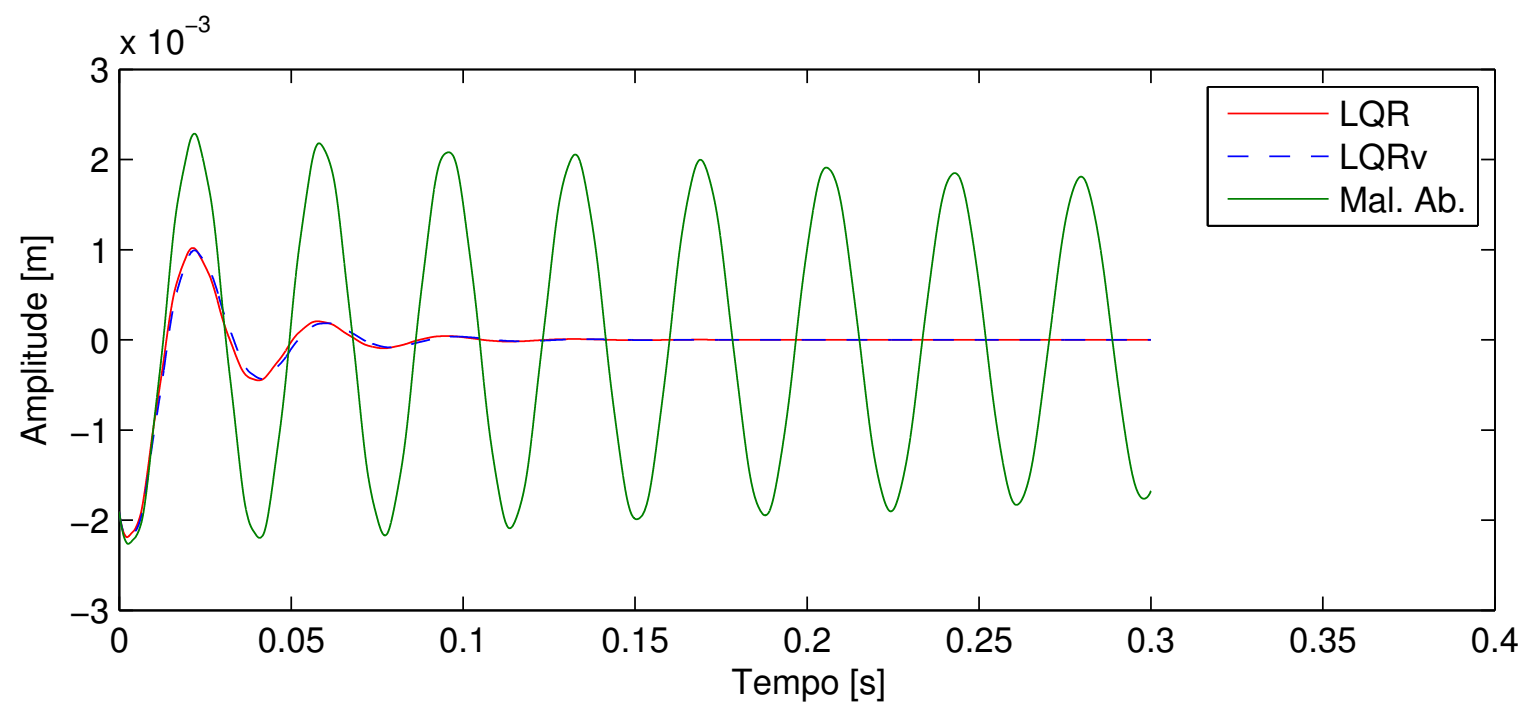

Figura 6 - Comparação entre as respostas temporais considerando a pior condição inicial do controle LQRv.

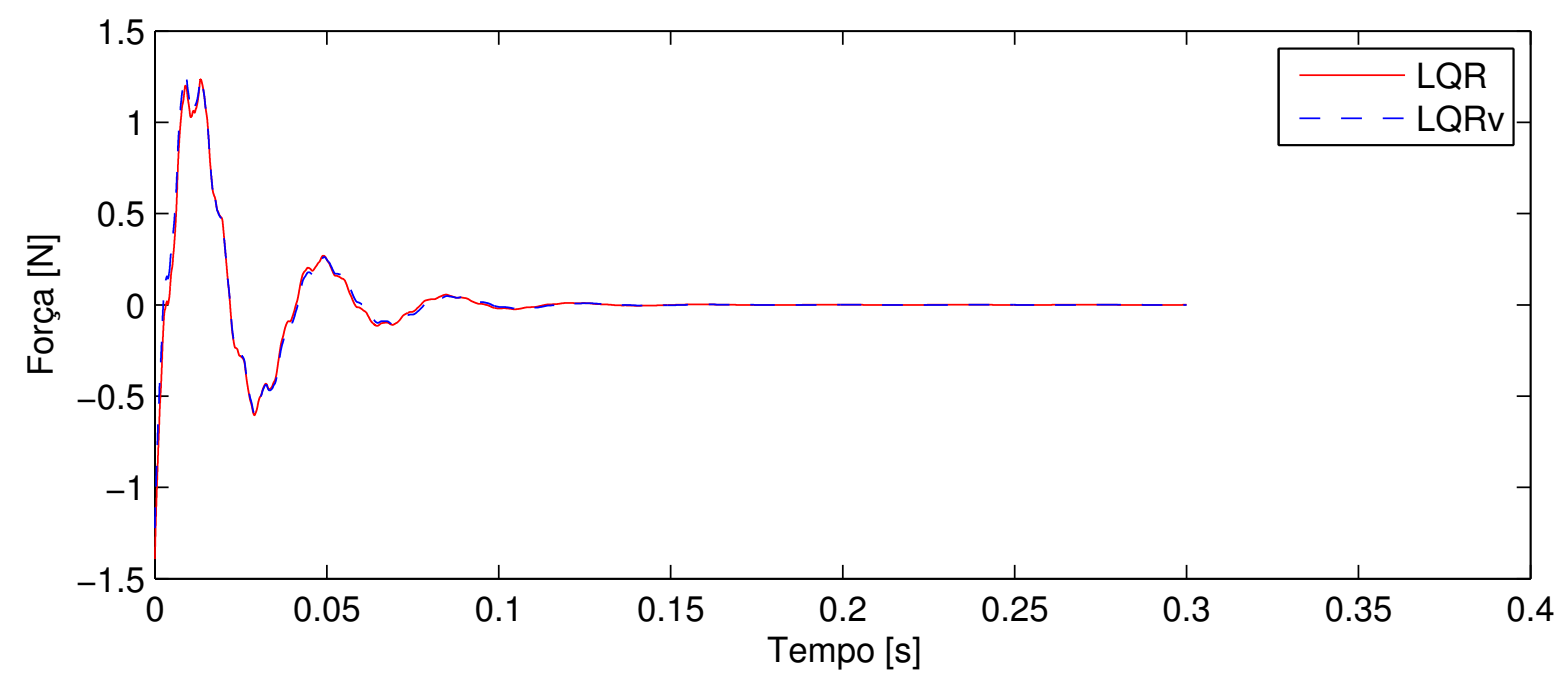

Figura 7 - Comparação entre os esforços de controle para a pior condição inicial do controle LQRv.

A partir da determinação da posição do atuador e especificação do tipo de sensor é possível projetar o sistema de controle com realimentação de saída. Inicialmente, para comparar a proposta de Levine e Athans com a sugerida na subseção 3.3.1, doravante intitulada "minmax" para simplificar a terminologia, considerou-se o controle colocalizado. Optou-se por esta configuração pois foi possível notar uma característica relevante com relação à dependência do valor inicial do algoritmo. Primeiramente, quando se considera o 
controle colocalizado com realimentação somente de velocidade, a função custo $J$ é função unicamente de um ganho escalar $k$. Conforme demonstrado na seção 3.2, para qualquer valor positivo de $k$ o sistema é estável. No caso de sistemas com amortecimento, é possível que o sistema ainda seja estável para um ganho negativo quando o termo à esquerda na equação (3.8) for maior que o termo à direita. No entanto, fica claro que a partir do momento em que o termo à direita for maior, o sistema não é estável, mostrando que o conjunto que contém os valores de ganho que estabilizam o sistema é conexo. Ademais, diminuindo o valor de $k$ a partir do mínimo global a função custo tende ao infinito, de modo que é possível esperar que não existam pontos extremos nessa região. A partir destas considerações, espera-se que este problema de otimização seja convexo, ou seja, que o ponto ótimo não dependa do valor inicial do algoritmo. De fato, esta característica foi observada nas simulações, conforme ilustrado na Figura 8. No gráfico, para cada valor do ganho $k$, foi utilizada a condição inicial que maximiza a razão entre os critérios de desempenho dos controladores.

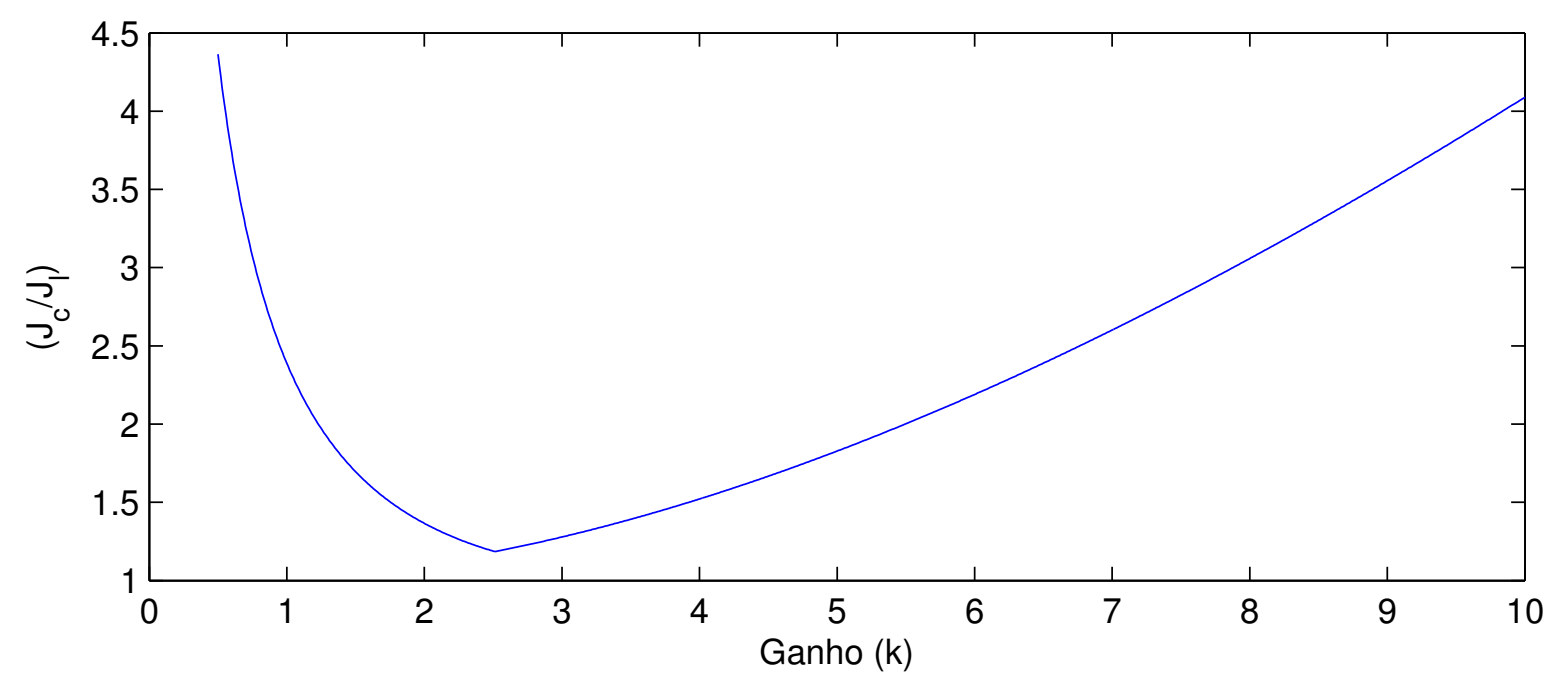

Figura 8 - Razão entre critérios de desempenho do controle colocalizado $\left(J_{c}\right)$ e do LQR $\left(J_{l}\right)$ em função do ganho de realimentação de velocidade.

Escolhida a configuração colocalizada, avaliam-se as propostas Levine-Athans e minmax para lidar com a dependência em relação a condição inicial. O ganho da proposta Levine-Athans é calculado a partir da minimização da função custo (3.32), substituindo a matriz que multiplica $P$ pela identidade, e para a proposta minmax utiliza-se o método descrito na subseção 3.3.1. Utilizando ambos procedimentos, obteve-se um ganho ótimo $k=0.89$ para a proposta dada por Levine-Athans e $k=2.51$ considerando a abordagem minmax. Comparando ambos controladores com o LQR utilizando a magnitude da função custo, verificou-se que para o minmax a diferença considerando a pior condição inicial foi de 18,62\%, enquanto que para Levine-Athans esta diferença atingiu 165,30\%. Na figura Figura 9 e na Figura 10, faz-se uma comparação entre ambos os controladores 
para a pior condição inicial da proposta de Levine-Athans. A diferença entre os critérios de desempenho nesse caso é de $91,11 \%$ a favor do minmax. Nestas figuras, nota-se que apesar de o esforço inicial para o controle obtido através da abordagem minmax ser maior, sua magnitude se anula mais rápido, pois a velocidade de resposta do sistema também é maior.

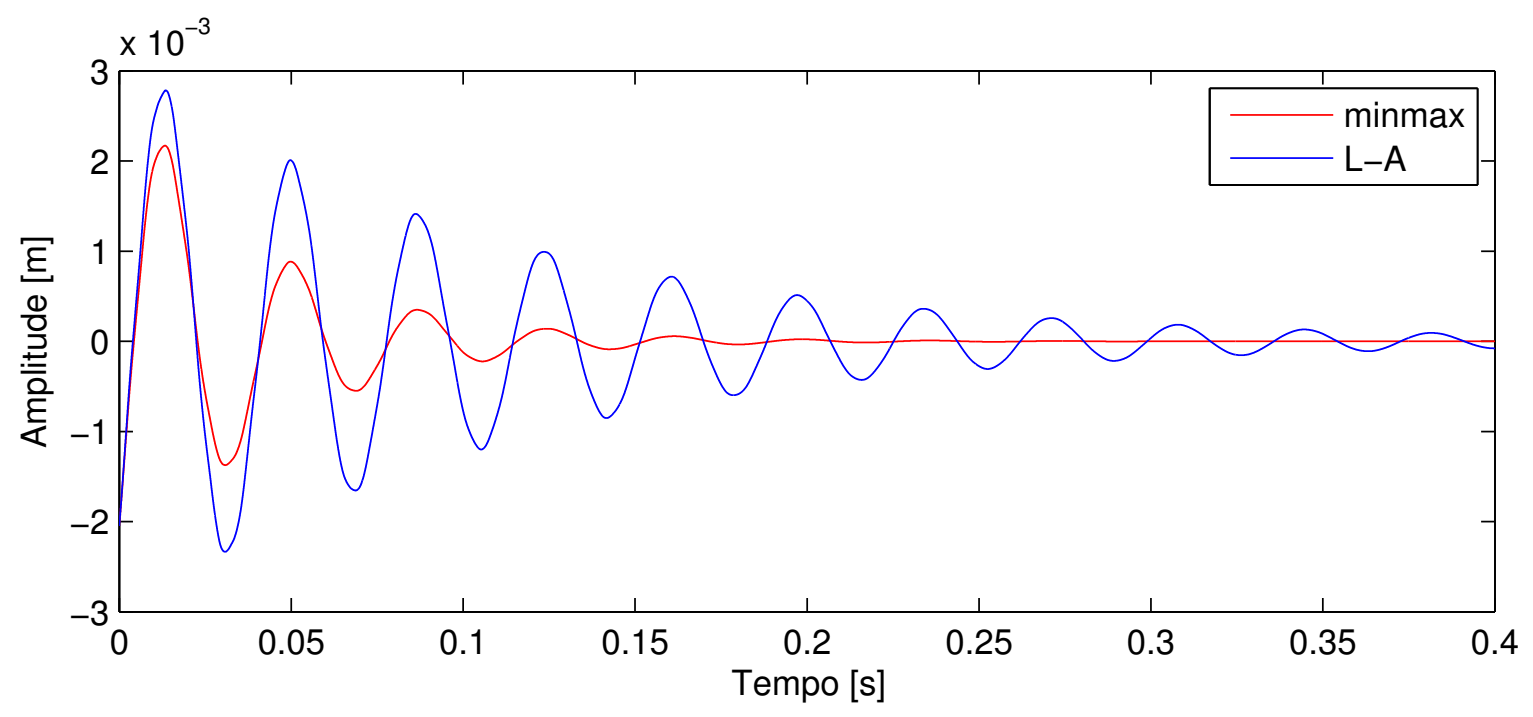

Figura 9 - Comparação entre as respostas temporais considerando a pior condição inicial da proposta Levine-Athans em relação ao LQR.

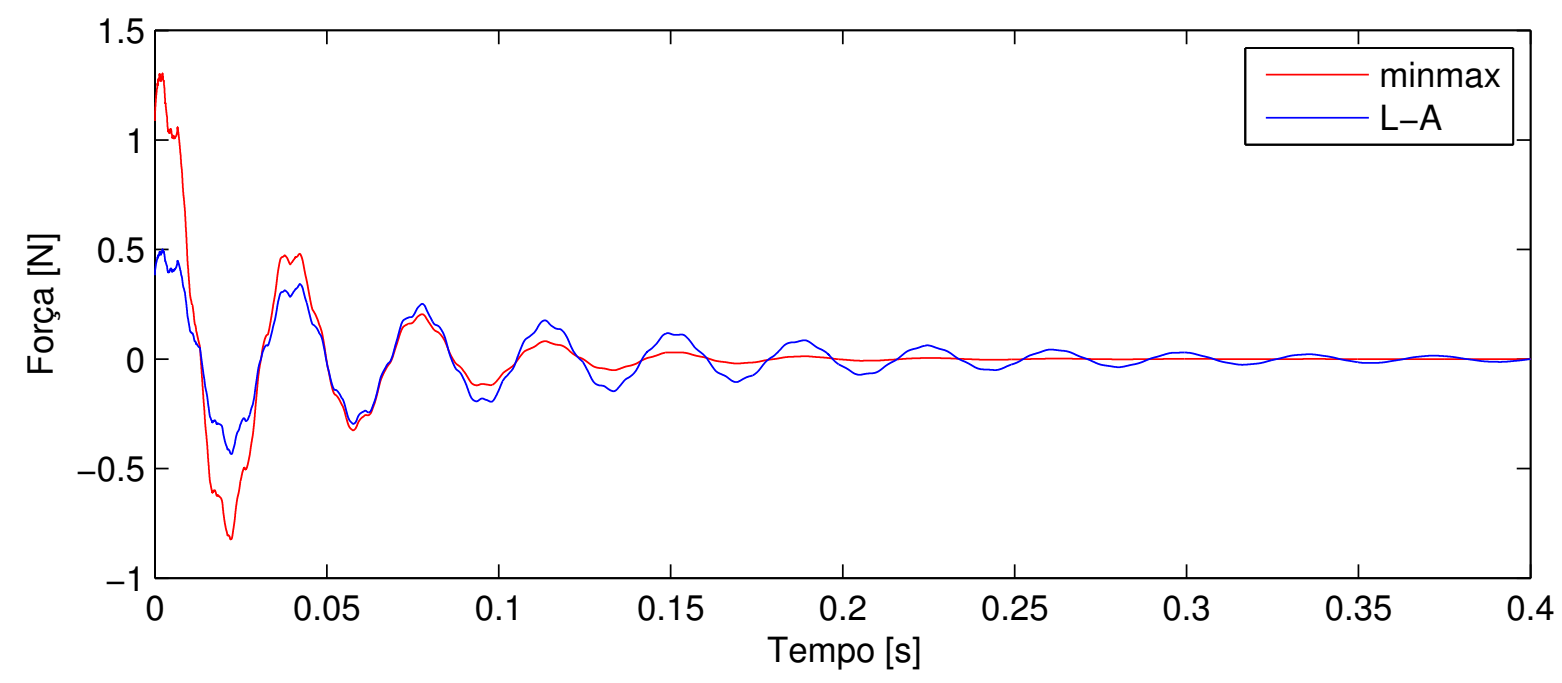

Figura 10 - Comparação entre os esforços de controle considerando a pior condição inicial da proposta Levine-Athans em relação ao LQR.

A abordagem minmax garante que o controlador obtido se aproxima mais do LQR que qualquer outro quando considerada a condição inicial que maximiza a razão entre as funções custo. No entanto, não há uma garantia do mesmo comportamento para outras condições iniciais. Para comparar as propostas minmax e Levine-Athans para outras 
condições iniciais, foi utilizada a base que diagonaliza simultaneamente as matrizes $P$ associadas à função custo de cada um dos controladores. Verificou-se que cada um dos controladores possuía um desempenho melhor para 10 condições iniciais dessa base, sendo que o controle baseado na proposta minmax possuía um desempenho melhor para condições iniciais associadas ao deslocamento e velocidade do primeiro ao quinto modo, enquanto o controle baseado na proposta Levine-Athans era melhor para os modos restantes. No entanto, para a condição inicial que mais favorecia a proposta minmax, a diferença entre os critérios de desempenho chegava a 124,59\%, enquanto que para a condição inicial que mais favorecia a proposta Levine-Athans, essa diferença era de somente 17,55\%. A razão entre a função custo associada ao Levine-Athans pela associada ao $\operatorname{minmax}\left(J_{c L A} / J_{c m m}\right)$ para cada uma das condições iniciais da base está indicada na Tabela 3, junto com o modo que prevalecia na condição inicial. Em geral, a velocidade de resposta da proposta minmax foi melhor para todos os modos. No entanto, para os modos de frequência mais alta não houve muita diferença no desempenho (Figura 11), enquanto o esforço de controle possuiu uma diferença considerável (Figura 12). Para a comparação entre as métricas consideradas, simulou-se uma das condições iniciais identificadas na Tabela 3 cuja razão $\left(J_{c L A} / J_{c m m}\right)$ foi de 1,01 .

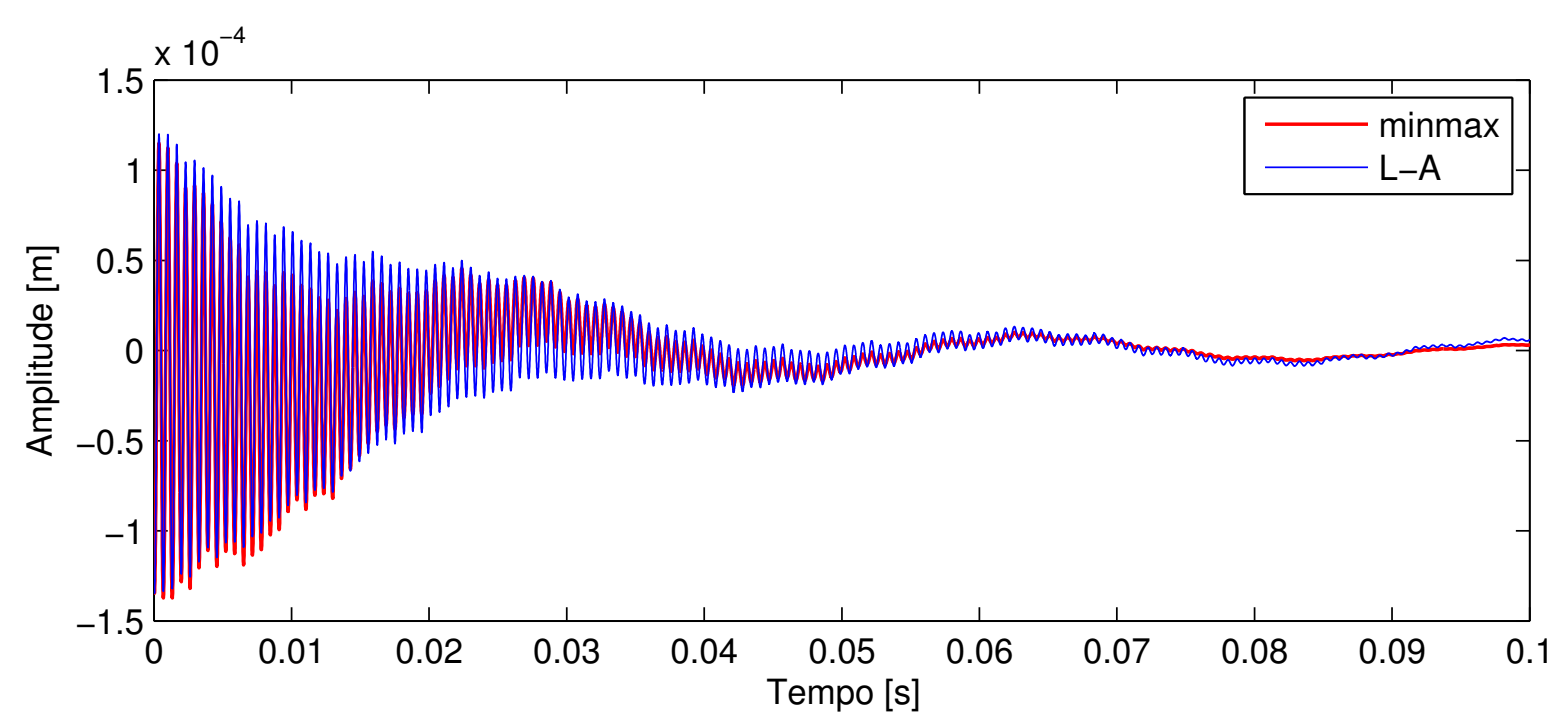

Figura 11 - Comparação entre as respostas temporais para uma condição inicial dada aproximadamente por uma velocidade inicial no quinto modo.

A última métrica utilizada para realizar uma comparação entre as duas propostas é a resposta em frequência. Como esta análise não considera o esforço empregado, o controle obtido através da proposta minmax obteve um desempenho melhor para todos os modos. A partir da Figura 13, nota-se que houve uma redução significativa de amplitude para os primeiros modos, principalmente para a proposta minmax. Como para os modos seguintes a redução de amplitude é baixa, optou-se por limitar a faixa de frequências no gráfico para uma melhor visualização da redução para os primeiros modos. No caso da proposta de 


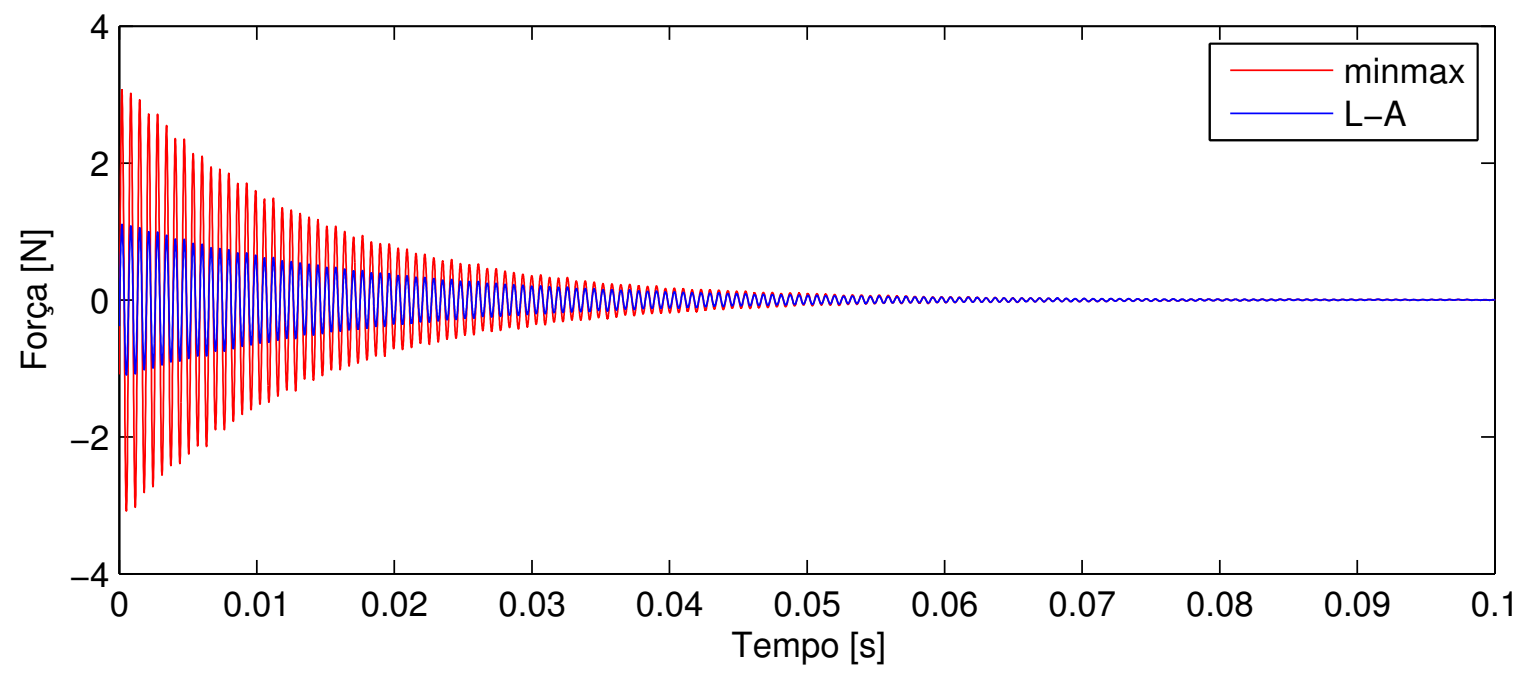

Figura 12 - Comparação entre os esforços de controle para uma condição inicial dada aproximadamente por uma velocidade inicial no quinto modo.

Tabela 3 - Comparação entre as funções custo para as propostas Levine-Athans e min$\max$.

\begin{tabular}{ccccccccccc}
\hline$\left(J_{c L A} / J_{c m m}\right)$ & 2,25 & 2,00 & 1,70 & 1,67 & 1,33 & 1,32 & 1,12 & 1,12 & 1,01 & 1,01 \\
\hline Modo & $1^{\circ}$ & $1^{\circ}$ & $2^{\circ}$ & $2^{\circ}$ & $3^{\circ}$ & $3^{\circ}$ & $4^{\circ}$ & $4^{\circ}$ & $5^{\circ}$ & $5^{\circ}$ \\
\hline & & & & & & & & & & \\
\hline$\left(J_{c L A} / J_{c m m}\right)$ & 0,95 & 0,95 & 0,85 & 0,85 & 0,91 & 0,91 & 0,87 & 0,87 & 0,89 & 0,88 \\
\hline Modo & $6^{\circ}$ & $6^{\circ}$ & $10^{\circ}$ & $10^{\circ}$ & $7^{\circ}$ & $7^{\circ}$ & $9^{\circ}$ & $9^{\circ}$ & $8^{\circ}$ & $8^{\circ}$ \\
\hline
\end{tabular}

Levine-Athans, a redução foi de $20,6 d B, 8,1 d B$ e $3,9 d B$ para os três primeiros modos, enquanto para a proposta minmax essa diferença foi de $29,2 d B, 14,3 d B$ e $8,3 d B$.

Após esta análise sobre a dependência em relação à condição inicial do sistema, estuda-se o caso em que são utilizados mais sensores não colocalizados. De início, faz-se uma observação sobre uma característica possivelmente relevante do ponto de vista prático sobre o método utilizado. Como o modelo utilizado para estrutura é contínuo, foi possível aplicar um método de busca linear para a otimização, o qual usualmente converge mais rápido que métodos heurísticos. Observou-se que o tempo necessário para a convergência dependia, além do número de sensores utilizados, do valor inicial do algoritmo. No caso em que foram utilizados somente dois sensores o tempo de convergência esteve sempre abaixo de um segundo. Aumentando a quantidade para até cinco sensores, este valor não passou de três segundos. Apesar de o problema ser não convexo e ser necessário a utilização de diferentes valores iniciais para o algoritmo, acredita-se a partir dos resultados obtidos que o tempo computacional necessário não é um fator desfavorável ao método.

Para o projeto do sistema de controle com realimentação de saída, foram considerados inicialmente dois sensores. Utilizando a proposta minmax e usando diferentes valores iniciais para o algoritmo, obteve-se um vetor de ganhos $\boldsymbol{k}=[3,08 \quad 1,54]$ e o vetor 

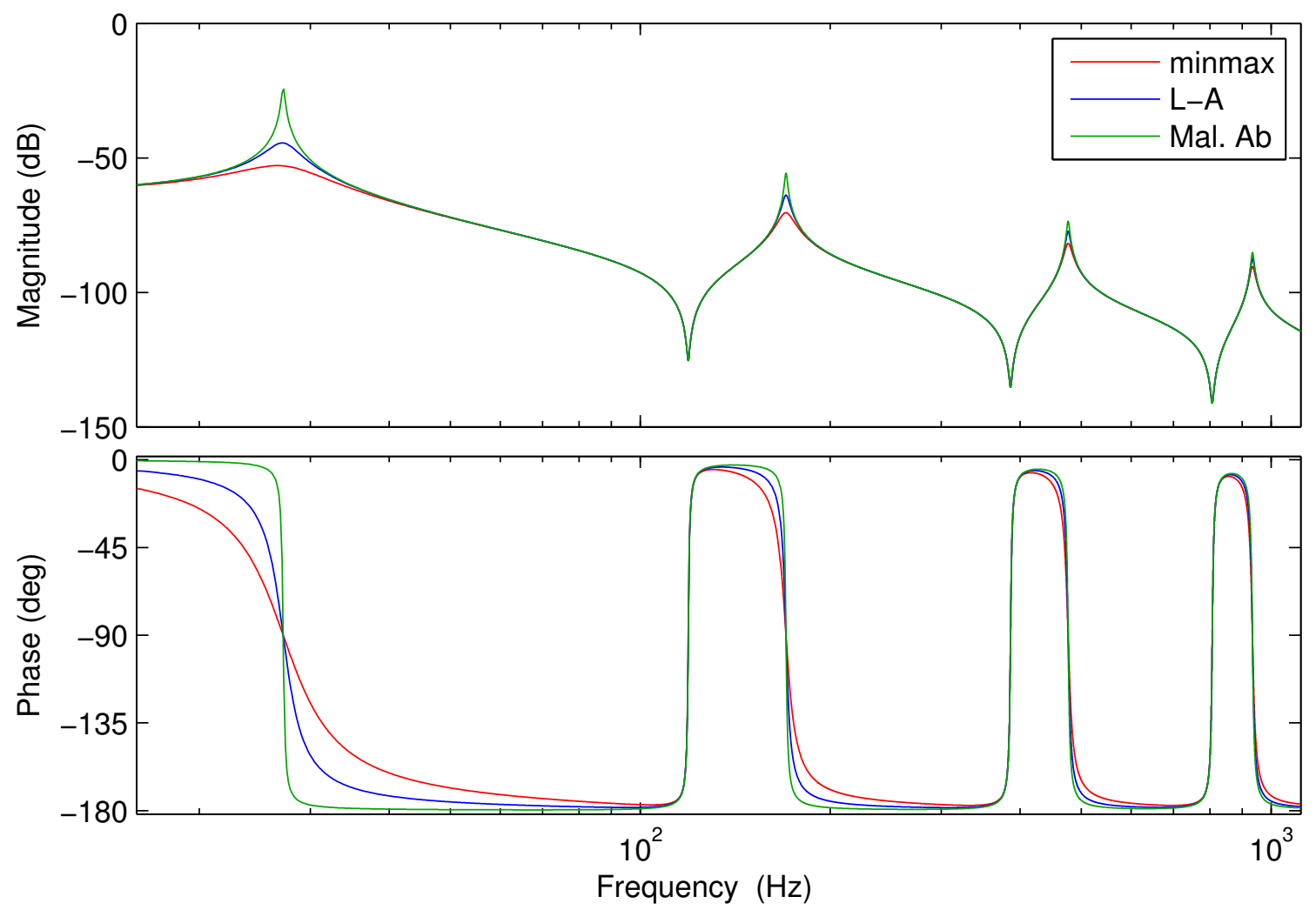

Figura 13 - Comparação entre as propostas de Levine-Athans e minmax através do diagrama de Bode.

de posições $\boldsymbol{\xi}=[282,8 \quad 300,0]$, sendo estas dadas em milímetros. A precisão utilizada para a solução pode ser questionável a partir da perspectiva experimental, no entanto, posteriormente na subseção 4.1.2 é realizado uma análise de sensibilidade à variação dos parâmetros, avaliando alterações no desempenho e estabilidade. A máxima diferença obtida entre as funções custo do controle com realimentação de saída com estes parâmetros e o LQR foi de somente 0,89\%, valor ligeiramente abaixo do LQR com os ganhos associados ao deslocamento anulados. Este valor revela um desempenho notável do método proposto, pois, considerando o pior caso, com somente dois sensores obteve-se praticamente o mesmo desempenho de um controlador ótimo que necessitaria de 20 sensores. Assim como na comparação entre a proposta minmax e a proposta Levine-Athans, também foi realizada uma comparação entre as funções custo da realimentação de saída com dois sensores $\left(J_{2 m m}\right)$ e do controle colocalizado $\left(J_{c m m}\right)$ para cada uma das condições iniciais da base que diagonaliza as matrizes $P$ associadas às funções custo. O resultado obtido está indicado na Tabela 4. Apesar da pequena diferença no desempenho, nota-se que o controle com dois sensores foi melhor ou igual para todas as condições iniciais consideradas. Na Figura 14 e Figura 15, exemplifica-se o que pode representar o valor de $18 \%$ para 
a máxima diferença entre as funções custo considerando a resposta temporal e esforço do controle. O segundo gráfico da figura Figura 15 é apenas uma ampliação do primeiro nos instantes iniciais da simulação.

Tabela 4 - Comparação entre as funções custo do controle colocalizado e controle com dois sensores utilizando a proposta minmax.

\begin{tabular}{ccccccccccc}
\hline$\left(J_{c m m} / J_{2 m m}\right)$ & 1,18 & 1,18 & 1,18 & 1,16 & 1,16 & 1,00 & 1,00 & 1,01 & 1,01 & 1,02 \\
\hline Modo & $1^{\circ}$ & $1^{\circ}$ & $10^{\circ}$ & $10^{\circ}$ & $9^{\circ}$ & $4^{\circ}$ & $4^{\circ}$ & $5^{\circ}$ & $5^{\circ}$ & $3^{\circ}$ \\
\hline$\left(J_{c m m} / J_{2 m m}\right)$ & 1,02 & 1,05 & 1,05 & 1,13 & 1,13 & 1,13 & 1,09 & 1,09 & 1,09 & 1,10 \\
\hline Modo & $3^{\circ}$ & $6^{\circ}$ & $6^{\circ}$ & $8^{\circ}$ & $8^{\circ}$ & $9^{\circ}$ & $2^{\circ}$ & $2^{\circ}$ & $7^{\circ}$ & $7^{\circ}$ \\
\hline
\end{tabular}

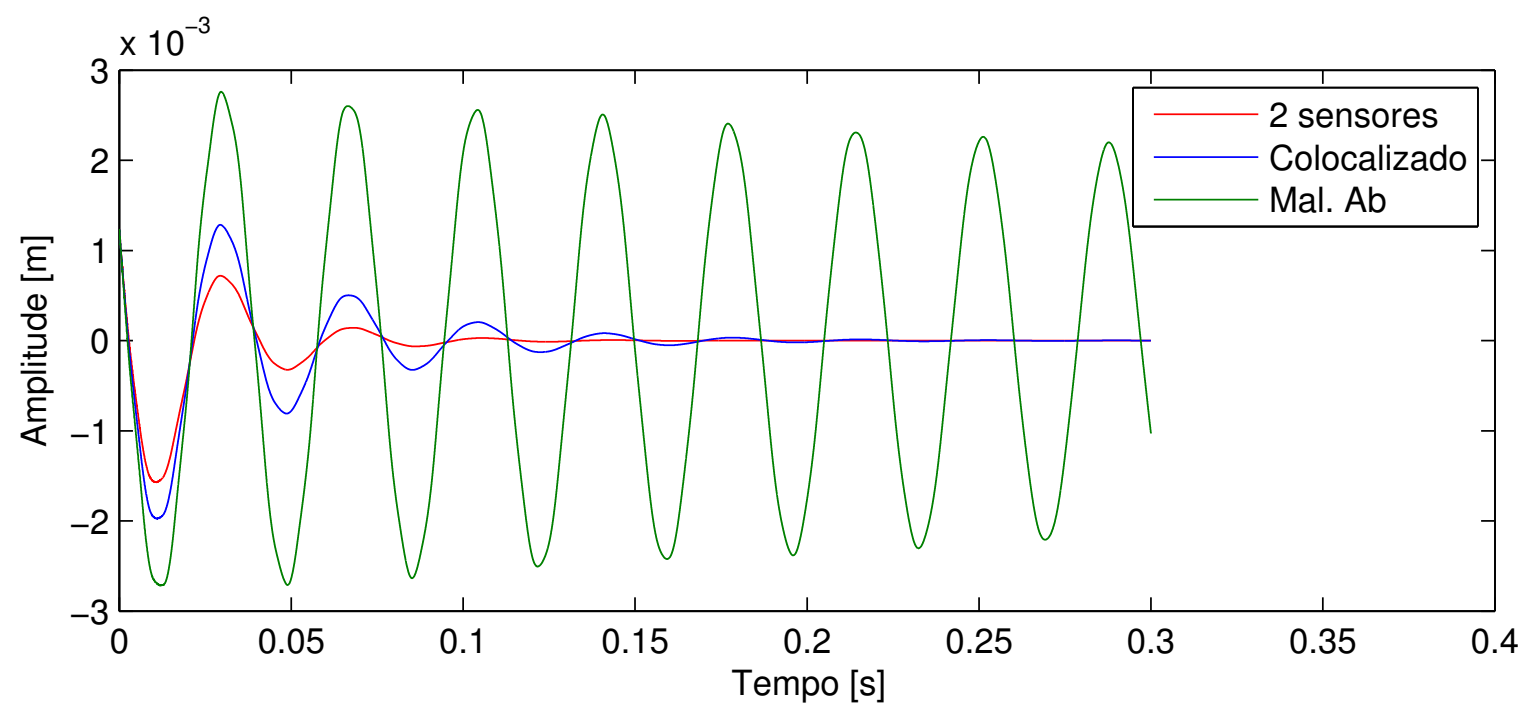

Figura 14 - Comparação entre as respostas temporais para uma condição inicial dada aproximadamente por uma velocidade inicial no primeiro modo.

A proximidade entre a magnitude das funções custo entre o controle de saída e o LQR também é um indicativo de um desempenho semelhante tanto no domínio do tempo quanto no domínio da frequência. De fato, este comportamento foi observado para todas as simulações realizadas. Um exemplo de resposta temporal para ambos os controladores é exibido na Figura 16 e na Figura 17. Um comportamento semelhante é observado para a resposta em frequência, conforme indicado na Figura 18, em que as curvas do controle com realimentação de saída e do LQR também estão praticamente sobrepostas. A redução de amplitude para os três primeiros modos nesse caso foi de $33,8 d B, 18,4 d B$ e $9 d B$.

Apesar da diferença entre o LQR e o caso com dois sensores ser praticamente desprezível, ainda foram investigados casos com um número maior de sensores. No entanto, a diferença observada foi insignificante. Para o caso com três sensores, a máxima diferença entre as funções custo entre o controle de saída e o LQR foi de 0,87\%. Aumentando para 

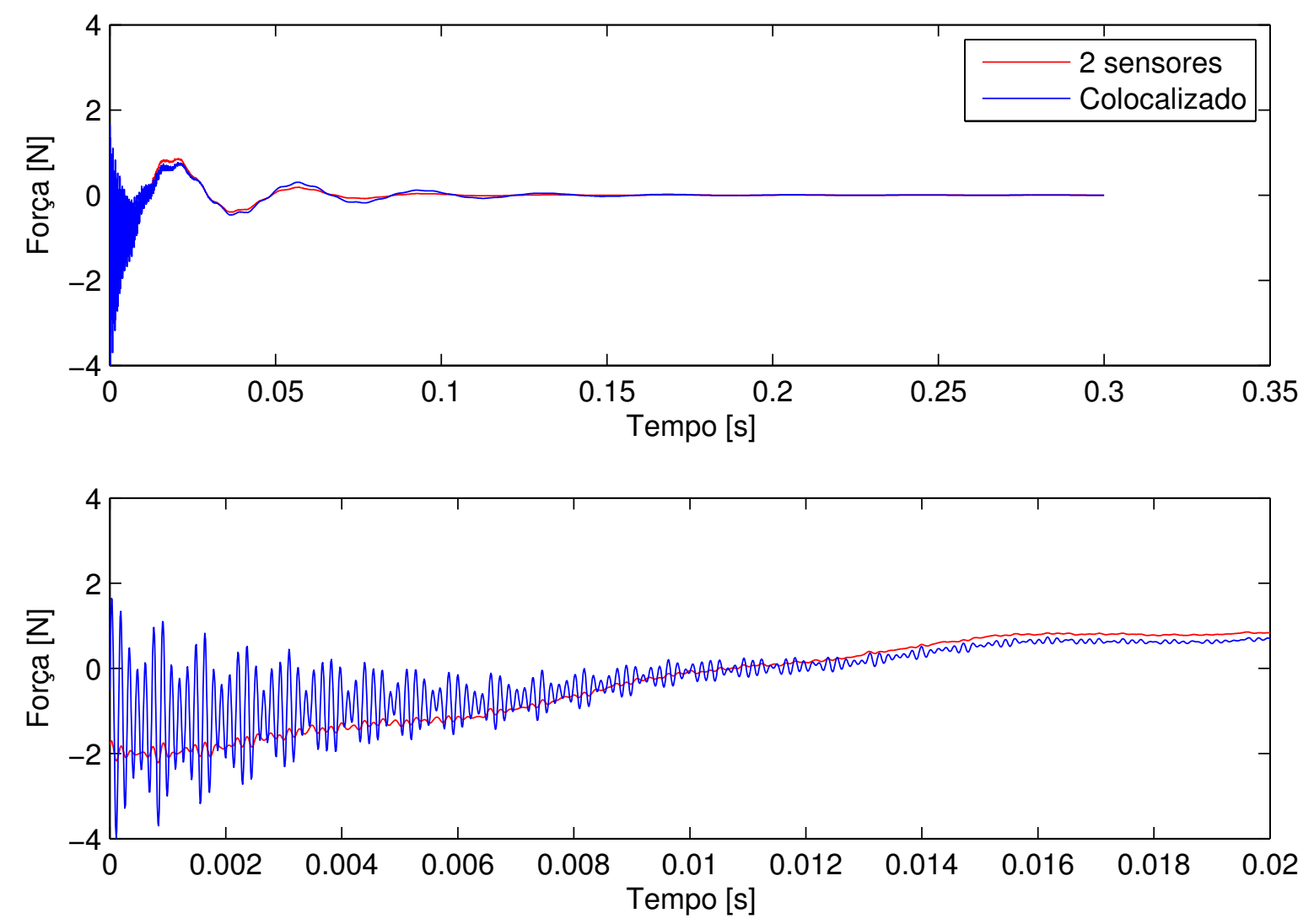

Figura 15 - Comparação entre os esforços de controle para uma condição inicial dada aproximadamente por uma velocidade inicial no primeiro modo.

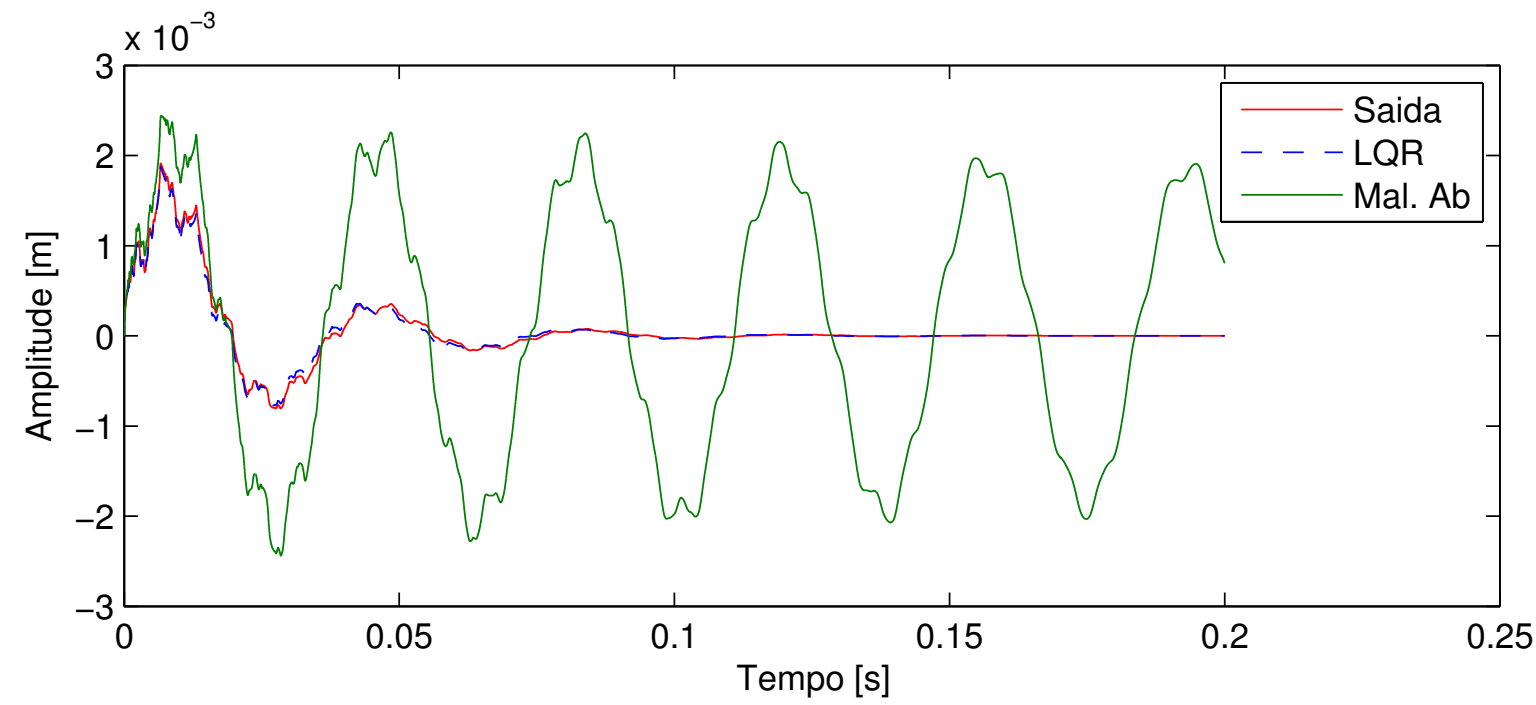

Figura 16 - Resposta temporal para o controle com realimentação de saída com dois sensores e o LQR, considerando uma entrada impulsiva aplicada na extremidade livre da viga.

o maior valor possível de sensores de velocidade, de 10 sensores, a diferença ainda se manteve em $0,87 \%$. 


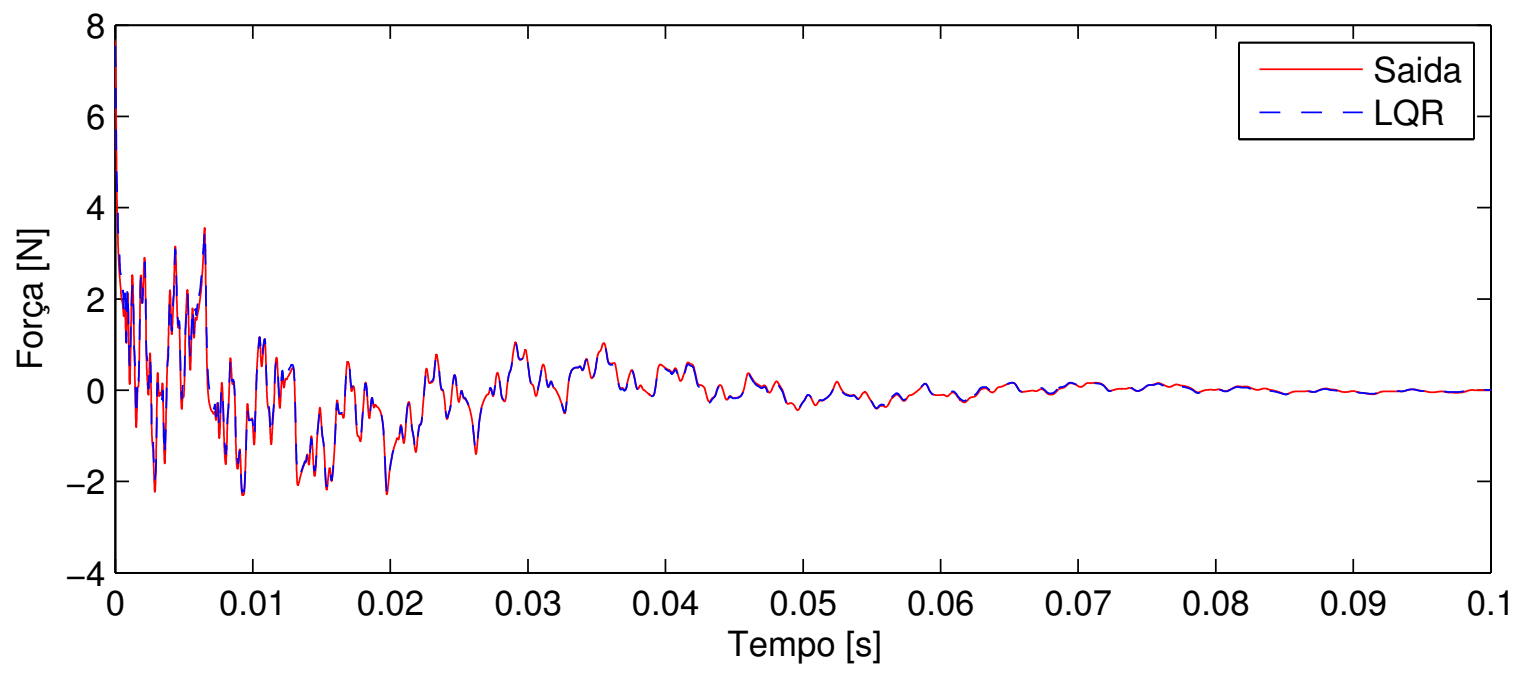

Figura 17 - Esforço de controle para o LQR e o controle com realimentação de saída com dois sensores, considerando uma entrada impulsiva aplicada na extremidade livre da viga.

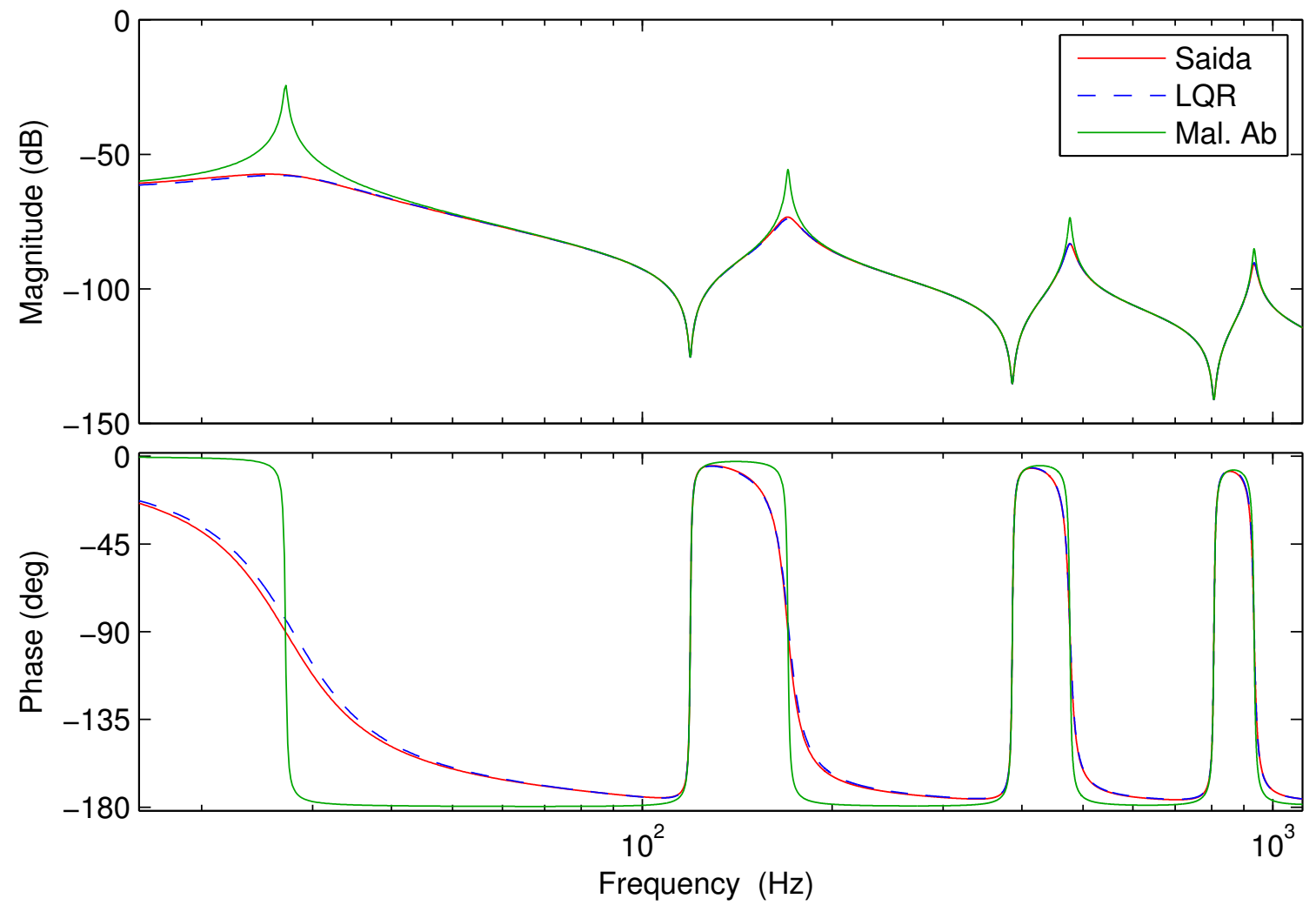

Figura 18 - Comparação entre o LQR e o controle com realimentação de saída com dois sensores através do diagrama de Bode. 
Os principais resultados deste exemplo, como a máxima diferença entre a função custo do controle de saída e o LQR, valores dos ganhos e posições dos sensores foram coligidos na Tabela 5 .

Tabela 5 - Principais resultados para a viga engastada-livre.

\begin{tabular}{ccccc}
\hline & Abordagem & Ganhos & Posições $(\mathrm{mm})$ & Máxima diferença para LQR \\
\hline \multirow{2}{*}{ Colocalizado } & Levine-Athans & $k=0,89$ & $\xi=300,0$ & $165,30 \%$ \\
& minmax & $k=2,51$ & $\xi=300,0$ & $18,62 \%$ \\
\hline \multirow{2}{*}{ Dois Sensores } & \multirow{2}{*}{$\operatorname{minmax}$} & $k_{1}=3,08$ & $\xi_{1}=282,8$ & $0,89 \%$ \\
& & $k_{2}=1,54$ & $\xi_{2}=300,0$ & 0 \\
\hline
\end{tabular}

\subsubsection{Variação de Parâmetros}

Conforme indicado na seção 2.3, o controle LQR possui robustez com relação à variação de parâmetros tanto da planta quanto do ganho de controle. De acordo com (ANDERSON; MOORE, 1971), espera-se que o controle com realimentação de saída possua as mesmas propriedades do LQR quando o valor esperado de suas funções custo se aproximarem. Nesta subseção, pretende-se fazer uma breve análise sobre a estabilidade e desempenho do sistema a partir da variação das posições e dos ganhos de controle em uma vizinhança do ponto ótimo.

Inicialmente, para avaliar as possíveis margens de alteração para os parâmetros do sistema, aplica-se novamente o método de Lyapunov. Utilizando a mesma função de Lyapunov definida em (3.7), e considerando o fato de que há somente um atuador na posição $\alpha$ e $n$ sensores de velocidade nas posições $\left(\xi_{1}, \ldots, \xi_{n}\right)$, chega-se na seguinte expressão para a derivada temporal de $V$ :

$$
\dot{V}=-2 \dot{\boldsymbol{\eta}}^{\top}\left(D+\sum_{i=1}^{n} k_{i} \boldsymbol{\phi}(\alpha) \boldsymbol{\phi}^{\top}\left(\xi_{i}\right)\right) \dot{\boldsymbol{\eta}}
$$

Portanto, a estabilidade do sistema está relacionada ao fato de a matriz entre parênteses na equação (4.10) ser positiva definida. Como a matriz $D$ representa o amortecimento intrínseco da estrutura e deve ser sempre positiva definida, a estabilidade do sistema depende do quanto as combinações entre as matrizes $\phi(\alpha) \phi^{\top}\left(\xi_{i}\right)$ alteram a positividade da matriz $D$. No caso em que há um número ímpar de nós entre o sensor na posição $\xi_{i}$ e o atuador na posição $\alpha$ para algum dos modos, a matriz $\phi(\alpha) \phi^{\top}\left(\xi_{i}\right)$ não pode ser positiva definida, já que pelo menos um dos elementos de sua diagonal é negativo. Neste caso, há uma faixa limitada de ganhos do sensor correspondente de modo a manter o sistema estável. No presente caso, em que a posição de um dos sensores é $\xi_{1}=282,8$, constatou-se que há um nó entre o sensor e o atuador do sétimo ao décimo modo. No entanto, ainda assim verificou-se que era possível manter o sistema estável para uma variação considerável a partir do ganho ótimo $\left(k_{1}=3,08\right)$. Para avaliar a estabilidade e desempenho do sistema 
variando os ganhos de cada um dos sensores, considerou-se uma faixa de 0,5 a 10 para cada um dos ganhos e calculou-se o máximo valor da razão entre as funções custo do controle de saída e o LQR para diferentes combinações possíveis. Os resultados obtidos estão indicados na Figura 19. Nota-se que a função custo cresce rapidamente quando os ganhos dos sensores tendem a zero.

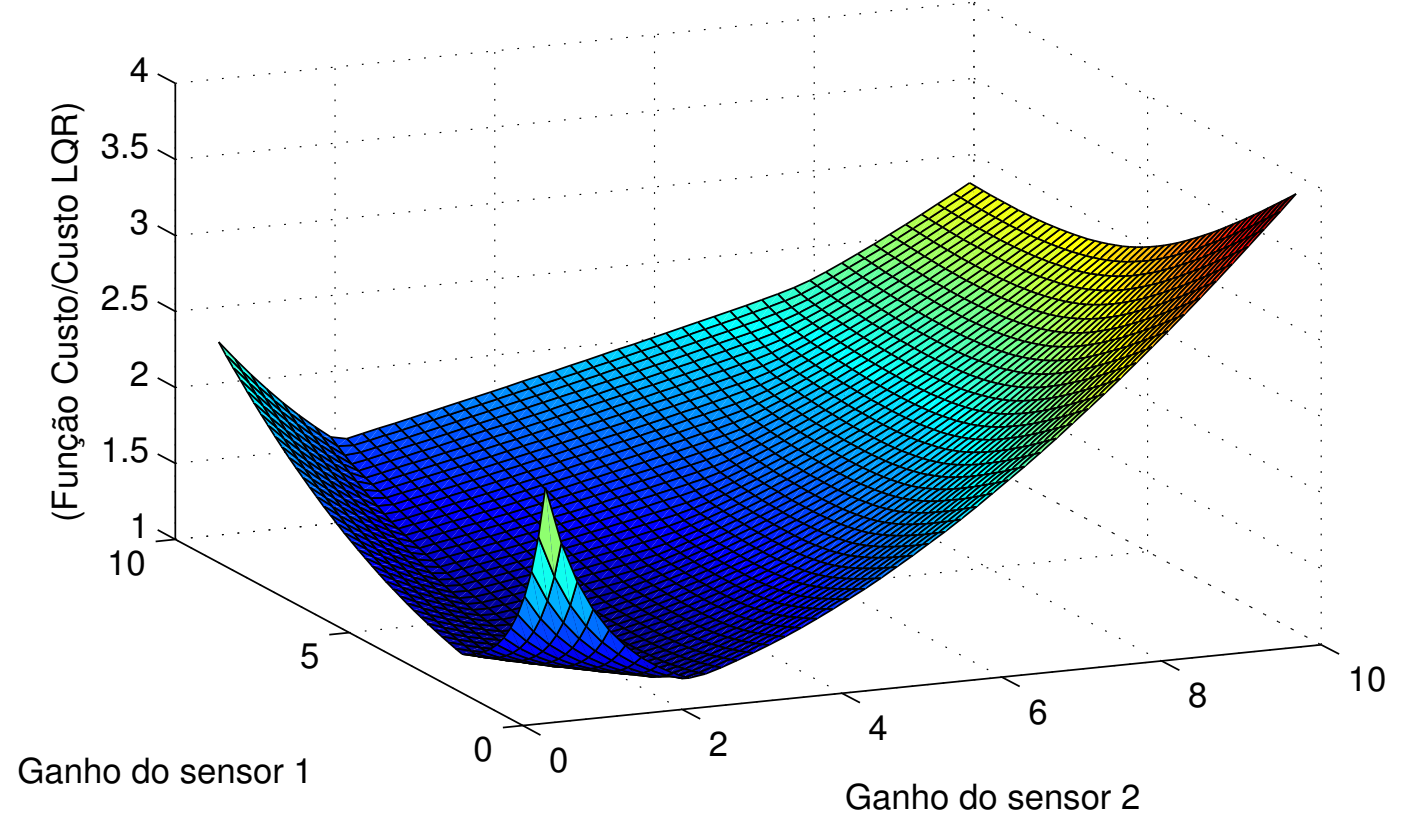

Figura 19 - Máximo valor da razão entre as funções custo do controle de saída e LQR quando os ganhos dos sensores são variados.

Uma análise semelhante foi realizada a partir de variações nas posições dos sensores. Considerou-se uma faixa de variação para cada um dos sensores de 250 a $300 \mathrm{~mm}$, e também foi gerada uma superfície comparando as funções custo do controle de saída e o LQR, a qual está indicada na Figura 20. Verificou-se que considerando os ganhos ótimos, o sistema é instável para valores de posições dos sensores próximos aos $240 \mathrm{~mm}$.

Além desses casos considerando variações separadas de ganhos e sensores, considerouse um caso de variação de $-3 \%$ nas posições dos sensores, de modo a obter as posições $\boldsymbol{\xi}=[274,3 \quad 291,0]$, e nos ganhos, obtendo os valores $\boldsymbol{k}=[2,98 \quad 1,49]$. Comparou-se este caso com o ponto ótimo a partir da aplicação de uma entrada impulsiva na extremidade livre da viga e os resultados estão indicados na Figura 21 e na Figura 22. Nota-se que mesmo para uma variação de aproximadamente $9 \mathrm{~mm}$ nas posições de ambos sensores ainda foi possível obter uma resposta semelhante ao ótimo.

Em seguida, realizou-se uma análise sobre o desempenho do controle com realimentação de saída variando a quantidade de modos considerados no modelo. O número de modos foi variado de 5 a 15 , sendo a frequência do $15^{\circ}$ modo de aproximadamente $16 \mathrm{kHz}$, e foram determinados os parâmetros ótimos para o controle colocalizado e o con- 


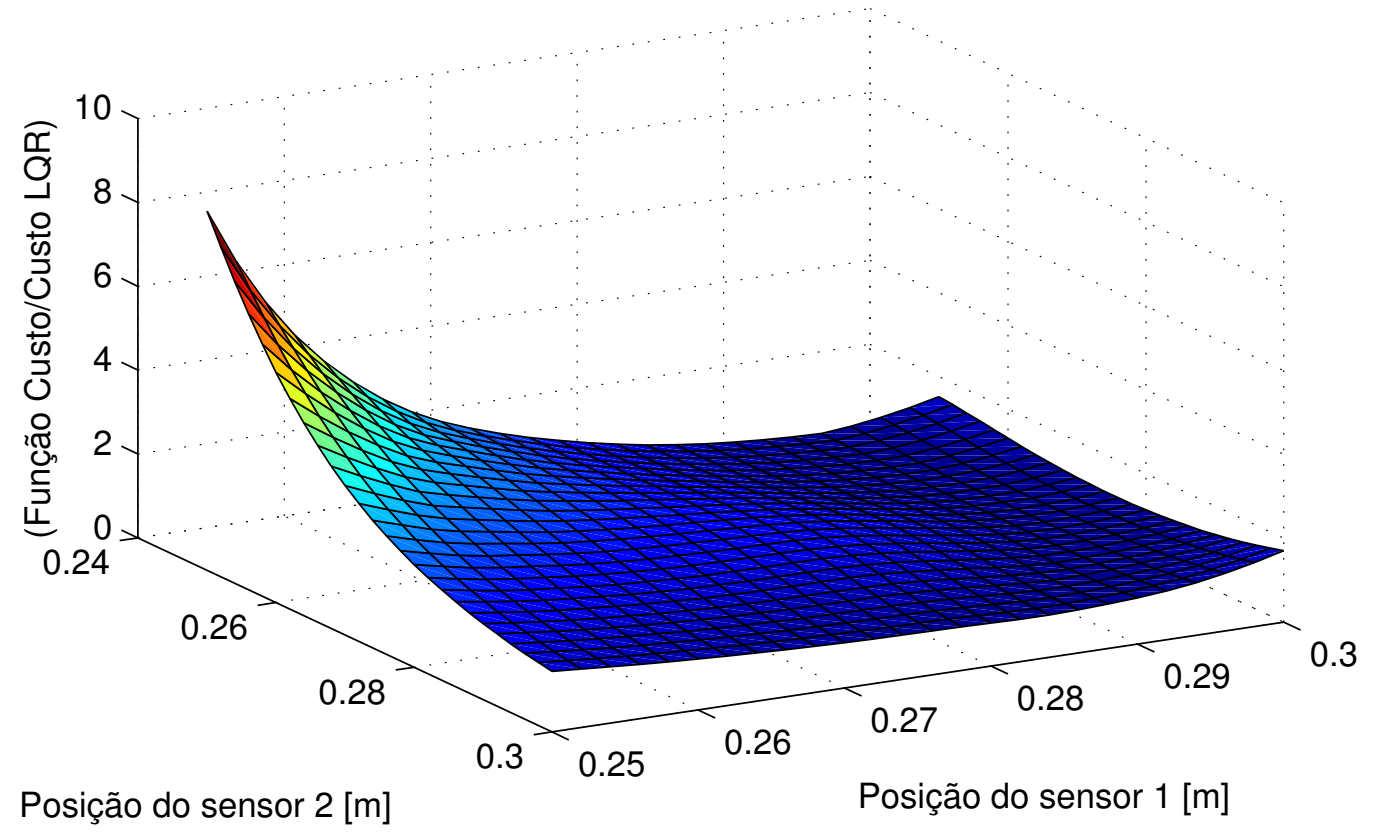

Figura 20 - Máximo valor da razão entre as funções custo do controle de saída e LQR quando as posições dos sensores são variadas.

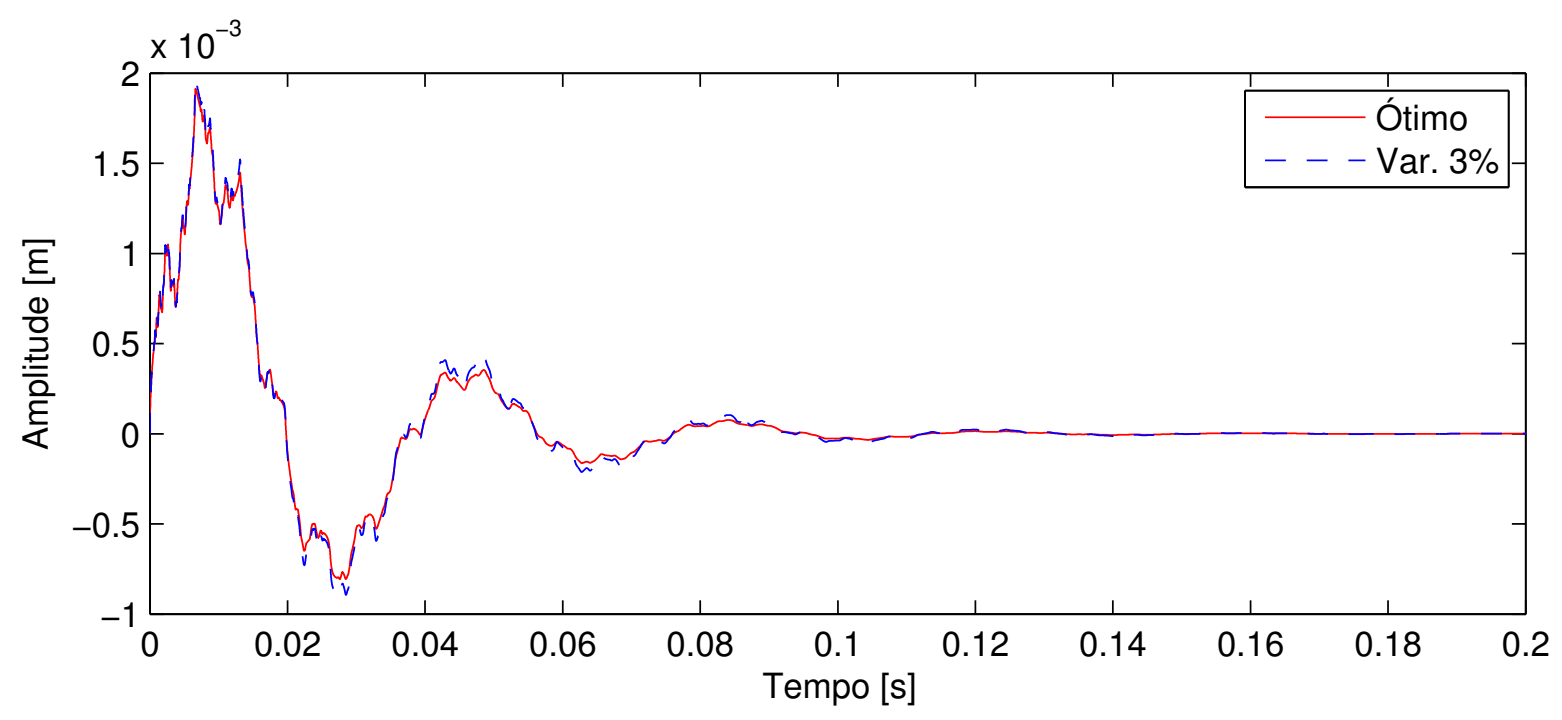

Figura 21 - Comparação entre as respostas temporais dos sistemas com parâmetros ótimos e com erro de $3 \%$ para uma entrada impulsiva na extremidade livre.

trole com dois sensores para cada uma das configurações. A máxima diferença entre as funções custo de cada um dos controles e o LQR também foi determinada. Os resultados obtidos estão indicados na Tabela 6 e na Tabela 7 .

O comportamento esperado para estes casos era o de um aumento na diferença de desempenho entre o controle com realimentação de saída e o LQR conforme o aumento do número de modos. Este resultado foi observado para o controle colocalizado, em que 


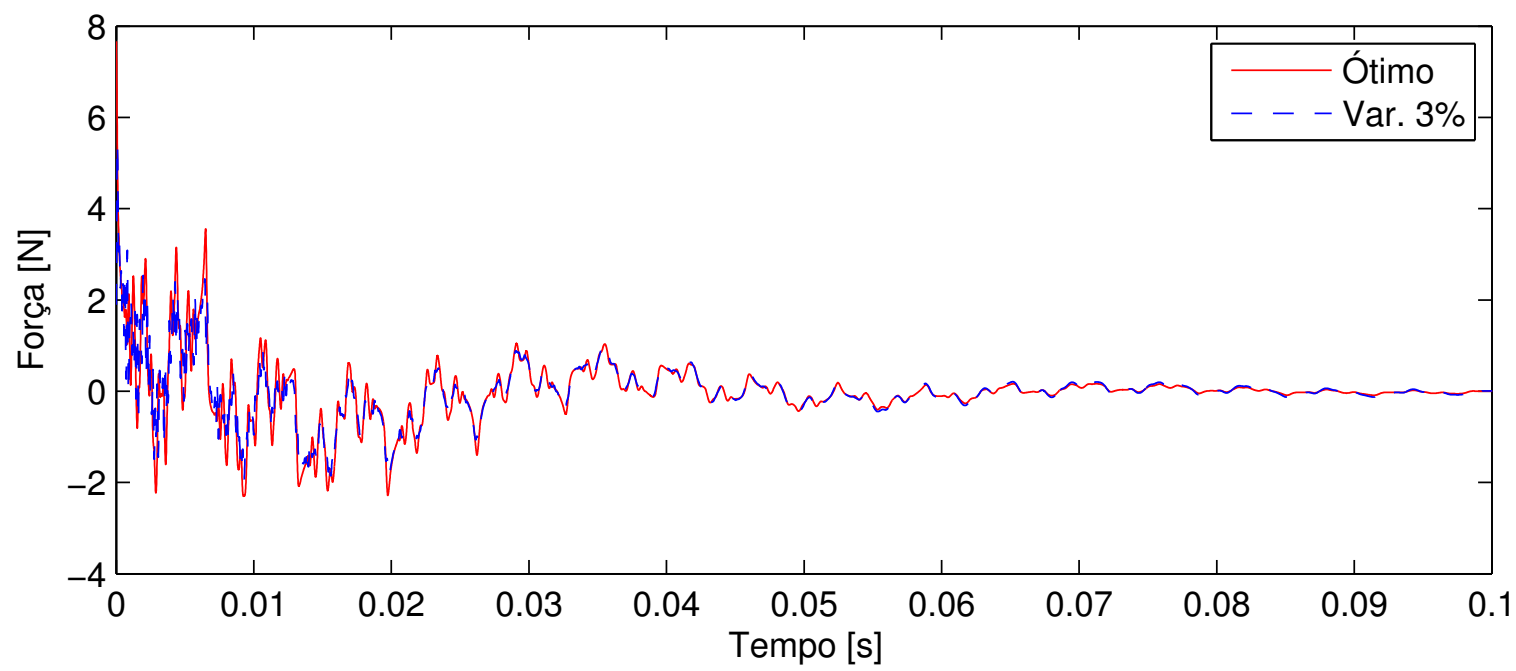

Figura 22 - Comparação entre os esforços de controle dos sistemas com parâmetros ótimos e com erro de $3 \%$ para uma entrada impulsiva na extremidade livre.

Tabela 6 - Comparação de desempenho entre o controle colocalizado e o LQR variando o número de modos.

\begin{tabular}{ccc}
\hline Número de modos & Ganho & Máxima diferença para LQR (\%) \\
\hline 5 & 3,17 & 6,97 \\
\hline 6 & 2,93 & 10,28 \\
\hline 7 & 2,76 & 13,12 \\
\hline 8 & 2,65 & 15,40 \\
\hline 9 & 2,57 & 17,19 \\
\hline 10 & 2,51 & 18,62 \\
\hline 11 & 2,47 & 19,78 \\
\hline 12 & 2,43 & 20,76 \\
\hline 13 & 2,40 & 21,60 \\
\hline 14 & 2,38 & 22,34 \\
\hline 15 & 2,36 & 22,99
\end{tabular}

notou-se também uma diminuição do ganho de acordo com o aumento do número de modos. Este comportamento não foi observado para o controle com realimentação de saída de dois sensores, em que a diferença de desempenho para o LQR foi praticamente a mesma independentemente do número de modos considerados. Além disso, verificou-se também uma mudança significativa dos parâmetros ótimos entre os sistemas com nove e dez modos.

Uma outra análise que pode ser realizada e que representa um problema importante para o controle não colocalizado, envolve a instabilização dos modos não considerados no modelo, fenômeno conhecido como spillover. Conforme indicado em (PREUMONT, 2011), considerando o controle LQG, o qual consiste em estimar todos os estados do modelo a partir de um sinal medido, é possível observar instabilidade no sistema a partir da inclusão 
Tabela 7 - Comparação de desempenho entre o controle com dois sensores e o LQR variando o número de modos.

\begin{tabular}{|c|c|c|c|}
\hline Número de modos & Posições $(\mathrm{mm})$ & Ganho & Máxima diferença para LQR (\%) \\
\hline 5 & $\begin{array}{l}\xi_{1}=288,0 \\
\xi_{2}=171,7\end{array}$ & $\begin{array}{c}k_{1}=4,77 \\
k_{2}=-0,35\end{array}$ & 0,87 \\
\hline 6 & $\begin{array}{l}\xi_{1}=287,7 \\
\xi_{2}=177,1\end{array}$ & $\begin{array}{c}k_{1}=4,80 \\
k_{2}=-0,37\end{array}$ & 0,87 \\
\hline 7 & $\begin{array}{l}\xi_{1}=287,7 \\
\xi_{2}=177,6\end{array}$ & $\begin{array}{c}k_{1}=4,80 \\
k_{2}=-0,37\end{array}$ & 0,87 \\
\hline 8 & $\begin{array}{l}\xi_{1}=287,8 \\
\xi_{2}=179,1\end{array}$ & $\begin{array}{c}k_{1}=4,80 \\
k_{2}=-0,37\end{array}$ & 0,87 \\
\hline 9 & $\begin{array}{l}\xi_{1}=288,5 \\
\xi_{2}=191,3\end{array}$ & $\begin{array}{c}k_{1}=4,77 \\
k_{2}=-0,31\end{array}$ & 0,87 \\
\hline 10 & $\begin{array}{l}\xi_{1}=300,0 \\
\xi_{2}=282,8\end{array}$ & $\begin{array}{l}k_{1}=1,54 \\
k_{2}=3,08\end{array}$ & 0,89 \\
\hline 11 & $\begin{array}{l}\xi_{1}=300,0 \\
\xi_{2}=281,5\end{array}$ & $\begin{array}{l}k_{1}=1,74 \\
k_{2}=2,88\end{array}$ & 0,89 \\
\hline 12 & $\begin{array}{l}\xi_{1}=300,0 \\
\xi_{2}=281,1\end{array}$ & $\begin{array}{l}k_{1}=1,79 \\
k_{2}=2,83\end{array}$ & 0,89 \\
\hline 13 & $\begin{array}{l}\xi_{1}=300,0 \\
\xi_{2}=281,0\end{array}$ & $\begin{array}{l}k_{1}=1,80 \\
k_{2}=2,82\end{array}$ & 0,89 \\
\hline 14 & $\begin{array}{l}\xi_{1}=300,0 \\
\xi_{2}=281,0\end{array}$ & $\begin{array}{l}k_{1}=1,81 \\
k_{2}=2,81\end{array}$ & 0,89 \\
\hline 15 & $\begin{array}{l}\xi_{1}=300,0 \\
\xi_{2}=281,0\end{array}$ & $\begin{array}{l}k_{1}=1,81 \\
k_{2}=2,81\end{array}$ & 0,89 \\
\hline
\end{tabular}

de somente um modo no modelo. No caso da metologia proposta neste trabalho, ao menos para o exemplo da viga engastada-livre, obteve-se um bom desempenho em relação ao fenômeno de spillover. Para realizar esta análise, utilizaram-se as posições e ganhos ótimos do sistema com 5 modos (linha 2 da Tabela 7) para o controle dos casos de 6 modos a 15 modos. Em todos os casos obteve-se um sistema estável em malha fechada. Em relação ao desempenho do sistema de controle, verificou-se que a razão entre a função custo do controle de saída e do LQR aumentava conforme o número de modos inseridos no modelo. Como exemplo, para o sistema com 10 modos obteve-se uma diferença máxima para o LQR de 7,04\%, enquanto para o sistema com 15 modos esta diferença foi de 39,61\%. Além disso, a partir da base que diagonaliza as matrizes $P$ associadas ao controle de saída e LQR, verificou-se que para condições iniciais dadas pelos primeiros modos a diferença para o LQR ainda era pequena, sendo o principal motivo do aumento na diferença o esforço de controle para os modos de alta frequência. Da Figura 23 a Figura 25 foram comparadas as respostas temporais e esforços de controle entre o LQR o controle com realimentação de saída utilizando o ponto ótimo do sistema com 5 modos, identificado como "O5", para condições iniciais dadas por modos de alta e baixa frequência. 

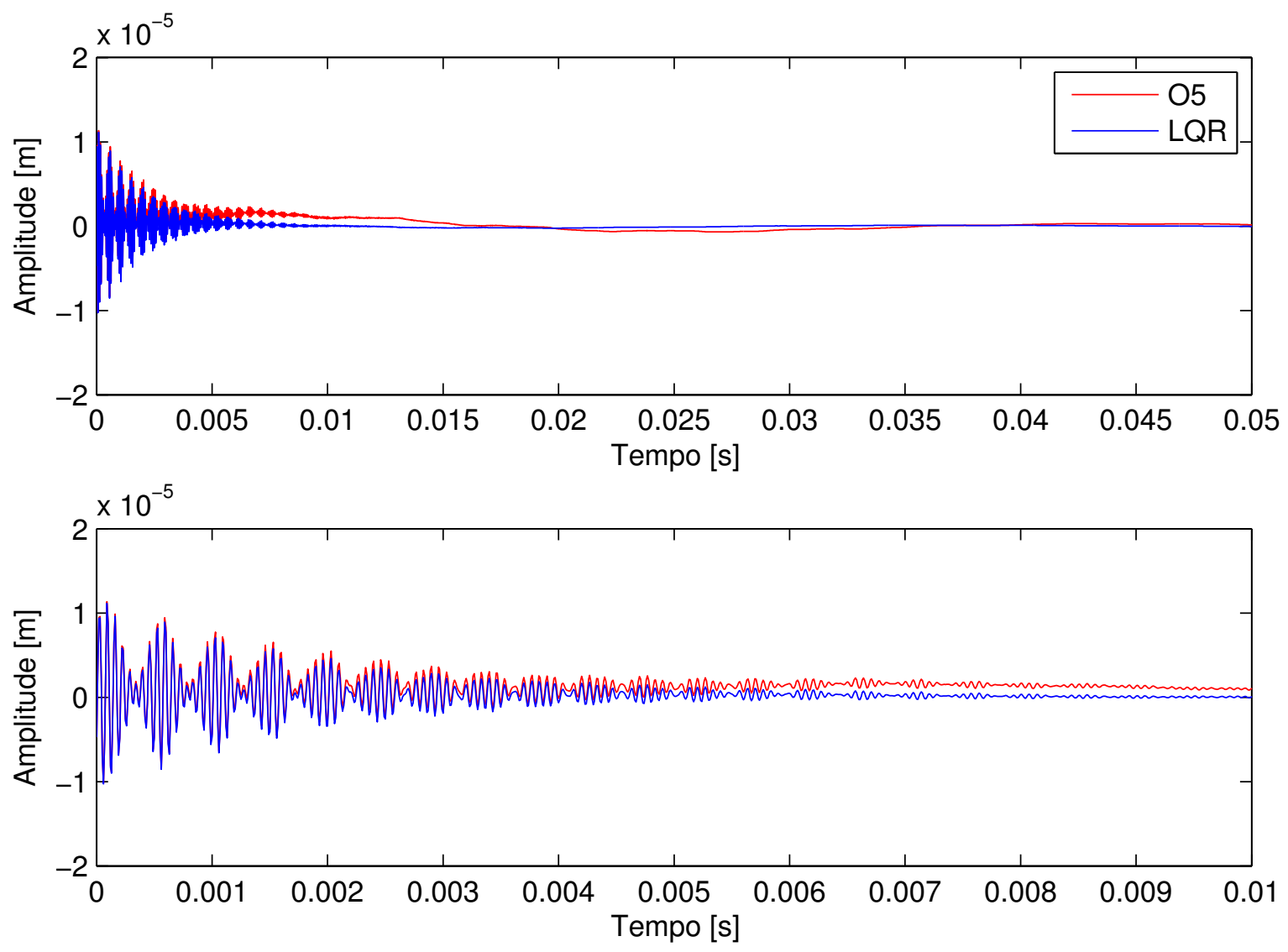

Figura 23 - Comparação das respostas temporais entre LQR e controle com realimentação de saída para uma condição inicial dada aproximadamente por uma velocidade inicial no $14^{\circ}$ modo.

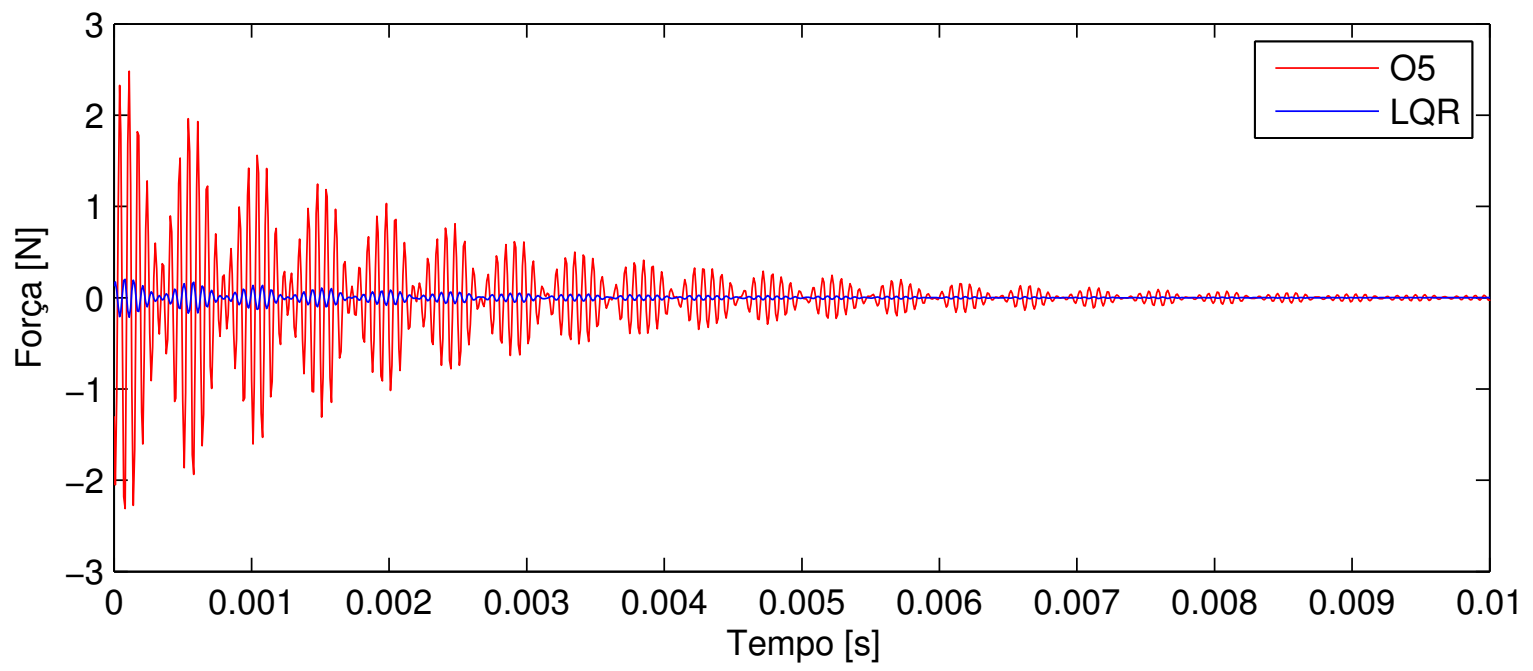

Figura 24 - Comparação dos esforços de controle entre LQR e controle com realimentação de saída para uma condição inicial dada aproximadamente por uma velocidade inicial no $14^{\circ}$ modo. 


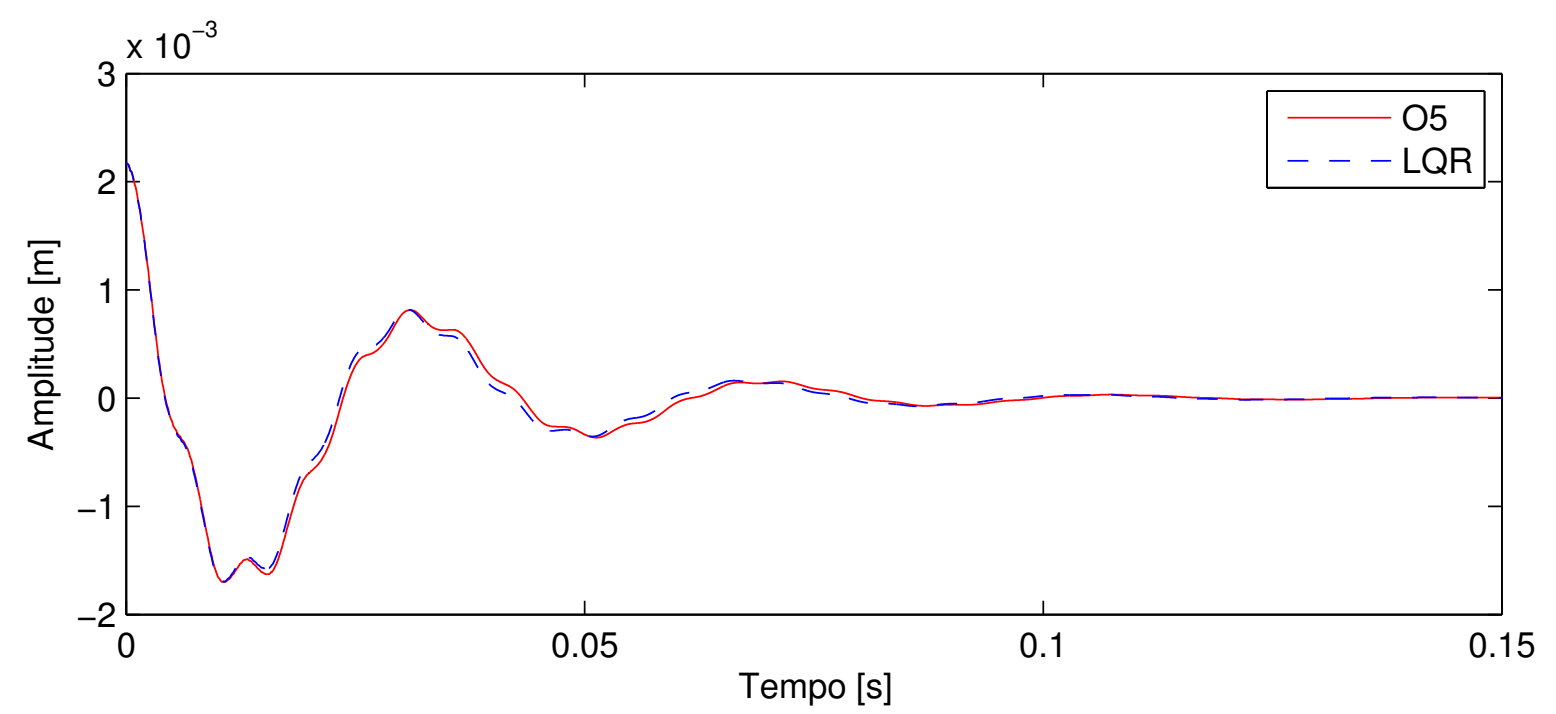

Figura 25 - Comparação das respostas temporais entre LQR e controle com realimentação de saída para uma condição inicial dada aproximadamente por uma velocidade inicial no $1^{\circ}$ modo.

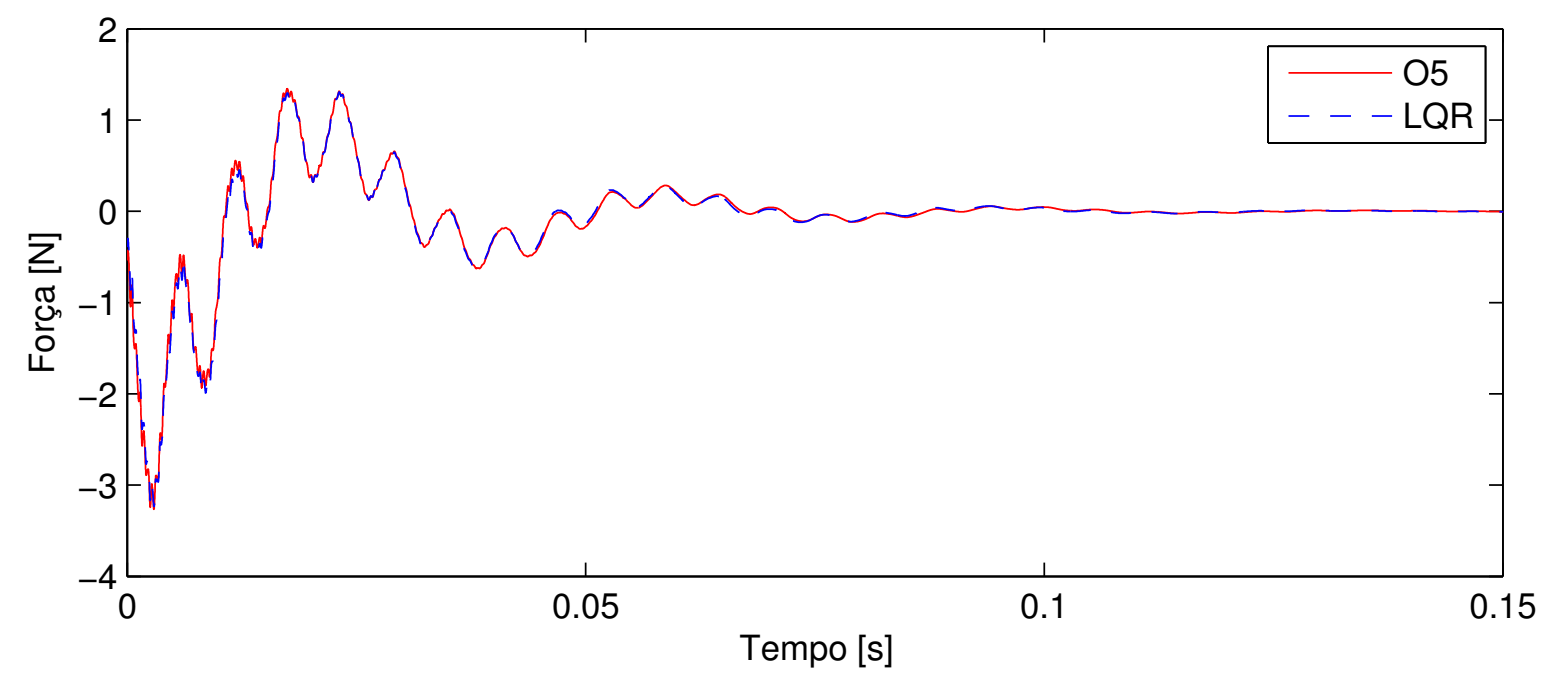

Figura 26 - Comparação dos esforços de controle entre LQR e controle com realimentação de saída para uma condição inicial dada aproximadamente por uma velocidade inicial no $1^{\circ}$ modo.

\subsection{Placa Simplesmente Apoiada}

Os resultados obtidos para a viga engastada-livre indicaram um bom desempenho da metodologia proposta. A fim de investigar se os bons resultados são decorrentes da simplicidade do modelo considerado, propõe-se o estudo de uma estrutura mais complexa, dada por um placa simplesmente apoiada em todos os lados (Figura 27). 


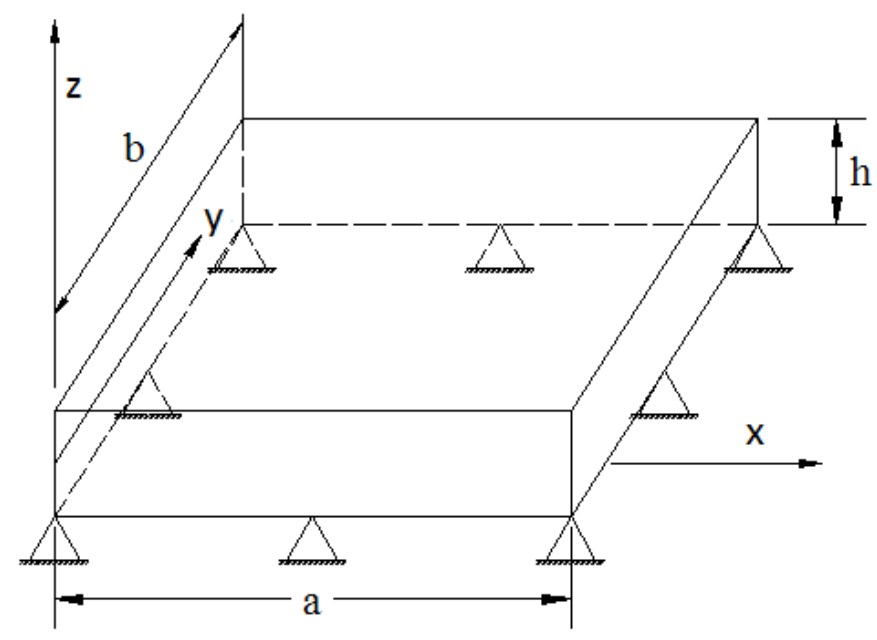

Figura 27 - Sistema de coordenadas e dimensões de uma placa retangular simplesmente apoiada em todos os lados.

\subsubsection{Modelo e Projeto do Sistema de Controle}

Para obtenção do modelo, é considerada a teoria de Kirchhoff-Love, a qual é baseada nas seguintes hipóteses (CRAIG; KURDILA, 2006):

- Durante a flexão da placa, existe um plano que não sofre deformação, o qual é denominado plano neutro;

- A deflexão de qualquer ponto da placa é pequena quando comparada a sua espessura;

- A tensão normal na direção perpendicular a placa $\left(\sigma_{z}\right)$ é desprezível quando comparada às tensões normais nas outras direções $\left(\sigma_{x}\right.$ e $\left.\sigma_{y}\right)$;

- A placa é feita de material homogêneo, isotrópico e linearmente elástico;

- Qualquer linha reta pertencente a placa e normal ao plano neutro permanece reta e normal ao plano durante a flexão.

Presumindo estas hipóteses e aplicando princípios variacionais ou as equações de Newton, obtém-se as equações do movimento assim como na equação (2.1), com $L$ dado por:

$$
L=D \nabla^{4}, \quad D=\frac{E h^{3}}{12\left(1-\nu^{2}\right)}
$$

em que $D$ é a rigidez à flexão da placa e $\nu$ é o coeficiente de Poisson do material. Para a placa simplesmente apoiada em todos os lados, o deslocamento em todos os pontos da 
fronteira são nulos, enquanto o momento na direção $x$ é nulo para os pontos $(x=0, a)$ e na direção $y$ para os pontos $(y=0, b)$. Logo, as condições de contorno podem ser escritas na forma (2.6):

$$
\begin{array}{lll}
B_{1}=1, & B_{2}=\frac{\partial^{2}}{\partial x^{2}}, & x=0, a \\
B_{1}=1, & B_{2}=\frac{\partial^{2}}{\partial y^{2}}, & y=0, b
\end{array}
$$

Assim como no caso da viga engastada-livre, a placa simplesmente apoiada também possui solução analítica. No entanto, também foi utilizado o método de elementos finitos. O intuito de utilizar o FEM para os modelos mais simples é exemplificar que a metodologia proposta também é compatível com esta técnica de modelagem. A placa foi discretizada em elementos retangulares iguais, com dimensões no eixo $x$ de $(a / 20)$ e no eixo $y$ de $(b / 20)$. Como funções de interpolação foram utilizadas as cúbicas de Hermite não conformes (2.53). Os parâmetros da placa estudada estão indicados na Tabela 8.

Tabela 8 - Características da placa estudada.

\begin{tabular}{lc}
\hline \hline Densidade $\left(\mathrm{kg} / \mathrm{m}^{3}\right)$ & 2700 \\
Módulo de Elasticidade $(\mathrm{GPa})$ & 69 \\
Coeficiente de Poisson & 0,33 \\
x-Comprimento $(\mathrm{mm})$ & 545 \\
y-Largura $(\mathrm{mm})$ & 400 \\
z-Altura $(\mathrm{mm})$ & 3 \\
\hline \hline
\end{tabular}

O procedimento utilizado para determinar as frequências naturais do modelo são semelhantes aos descritos para a viga. Foi considerada uma faixa de frequência de $1 \mathrm{kHz}$, o que implicou em um truncamento do modelo para os 18 primeiros modos. No caso da placa, as frequências teóricas são dadas pela equação (4.13). Uma comparação entre as frequências teóricas e as obtidas pelo modelo de elementos finitos está indicada na Tabela 9. Adicionalmente, também foi considerado um fator de amortecimento de 0,5\% para cada um dos modos.

$$
\omega_{m n}=\pi^{2}\left[\left(\frac{m}{a}\right)^{2}+\left(\frac{n}{b}\right)^{2}\right] \sqrt{\frac{D}{\rho h}}
$$

Dado o modelo da estrutura, contempla-se o projeto do controle LQR. Diferentemente do caso da viga engastada-livre, a escolha da posição do atuador para a placa simplesmente apoiada não é uma tarefa trivial. Conforme exibido na Figura 28, em que estão indicados somente os primeiros quatro modos para exemplificar, não há um ponto em que todos os modos assumem valor máximo. Neste caso, conforme já mencionado na 
Tabela 9 - Comparação entre os valores de frequência teóricos e os obtidos pelo modelo em elementos finitos.

\begin{tabular}{cccc}
\hline \hline Modo & Frequência Teórica $(\mathrm{Hz})$ & Frequência Modelo $(\mathrm{Hz})$ & Erro $(\%)$ \\
\hline $1^{\circ}$ & 70,06 & 70,01 & 0,06 \\
$2^{\circ}$ & 143,64 & 143,48 & 0,11 \\
$3^{\circ}$ & 206,65 & 206,46 & 0,09 \\
$4^{\circ}$ & 266,27 & 265,93 & 0,13 \\
$5^{\circ}$ & 280,23 & 279,53 & 0,25 \\
$6^{\circ}$ & 402,86 & 401,37 & 0,37 \\
$7^{\circ}$ & 434,31 & 433,88 & 0,10 \\
$8^{\circ}$ & 437,96 & 437,37 & 0,13 \\
$9^{\circ}$ & 507,89 & 506,24 & 0,33 \\
$10^{\circ}$ & 574,55 & 572,03 & 0,44 \\
$11^{\circ}$ & 630,52 & 626,98 & 0,56 \\
$12^{\circ}$ & 658,70 & 657,82 & 0,13 \\
$13^{\circ}$ & 753,03 & 752,28 & 0,10 \\
$14^{\circ}$ & 795,29 & 791,51 & 0,48 \\
$15^{\circ}$ & 802,21 & 796,21 & 0,75 \\
$16^{\circ}$ & 826,61 & 823,64 & 0,36 \\
$17^{\circ}$ & 928,49 & 927,29 & 0,13 \\
$18^{\circ}$ & 949,24 & 942,78 & 0,68 \\
\hline \hline
\end{tabular}

seção 4.1, a posição do atuador será determinada de modo a minimizar a função custo quadrática. Para lidar com a dependência em relação à condição inicial, são comparadas as propostas de Levine-Athans e a sugerida na subseção 3.3.2, doravante chamada de "robusta".

O primeiro passo da abordagem robusta é determinar um conjunto de possíveis condições inciais para o sistema. Para a placa simplesmente apoiada, é utilizada a mesma proposta de conjunto dada na seção 4.1. Inicialmente, determina-se o ponto da estrutura $\boldsymbol{x}_{p}$ que maximiza $w$ na equação (4.6). Utilizando o método SQP, determinou-se o ponto

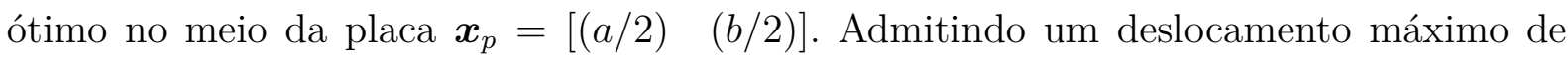
$2 \mathrm{~mm}$ para esse ponto, para ser coerente com a hipótese de pequenos deslocamentos, calculou-se uma força estática necessária de 153, $7 \mathrm{~N}$. Aplicando esta força sobre todos os pontos da placa e calculando o máximo deslocamento modal para cada um dos modos, chegou-se no seguinte vetor de deslocamentos modais:

$$
\begin{gathered}
\boldsymbol{\eta}_{M}=\left[\begin{array}{ccccccccccc}
11873 & 2827 & 1365 & 823 & 745 & 361 & 309 & 304 & 227 & 178 & \ldots \\
148 & 134 & 103 & 93 & 92 & 86 & 68 & 65
\end{array}\right]^{\top} \times 10^{-7} \\
\end{gathered}
$$

Assumindo velocidades iniciais nulas, chega-se em um vetor $\boldsymbol{\Delta}$ na forma (4.8). Nota-se a partir da equação (4.7) e da equação (4.14) que esta abordagem prioriza os modos de baixa frequência, o que decorre da utilização de uma força estática. Para de- 
$1^{\circ}$ modo

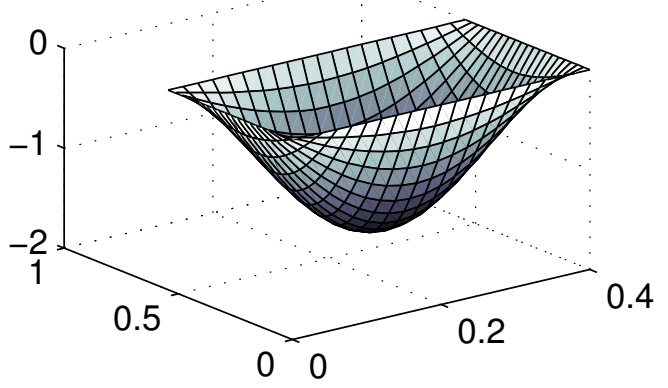

3ำ modo

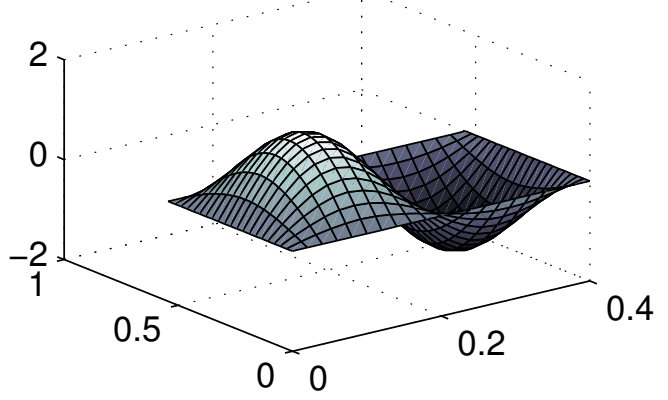

$2^{\circ}$ modo

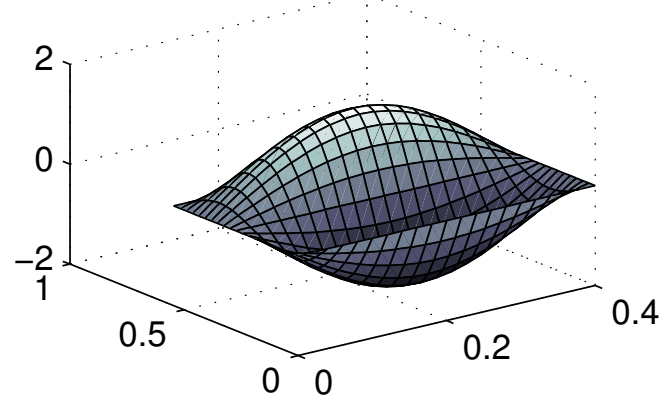

4ํㅡodo

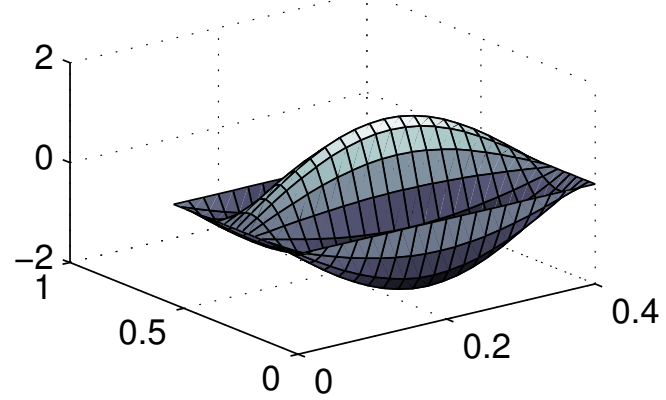

Figura 28 - Modos de vibrar para uma placa simplesmente apoiada.

terminar completamente a função custo, ainda é necessário especificar as matrizes de ponderação. No caso da ponderação dos estados, adotou-se novamente a matriz dada em (4.9). Para a matriz $R$, após algumas simulações escolheu-se o valor 0, 001.

Dados estes parâmetros, pode-se determinar a posição do atuador. Para a proposta robusta, obteve-se a posição $\boldsymbol{\alpha}_{r}=[211,6 \quad 162,9]$, enquanto para Levine-Athans a posição obtida foi $\boldsymbol{\alpha}_{L A}=\left[\begin{array}{ll}464,9 & 67,7\end{array}\right]$. Inicialmente, as duas propostas foram comparadas de acordo com a magnitude da função custo considerando diferentes condições iniciais. Uma vantagem desta métrica em relação à resposta em frequência é o fato desta não depender de posições de excitação e medição. As condições iniciais utilizadas são dadas pela base que diagonaliza as matrizes $P$ associadas a ambas funções custo, de modo que é possível determinar a máxima diferença de desempenho de ambas propostas. Diferentemente dos casos estudados na viga engastada-livre, cada uma das condições iniciais deste caso possuíam componentes relevantes de diversos modos. Apesar de não ser possível identificar um modo predominante na condição inicial, optou-se por exibir os valores entre as razões das funções custo a fim de mostrar o desempenho de cada uma das propostas para condições distintas. Os resultados obtidos estão indicados na Tabela 10, em que as condições iniciais foram enumeradas de 1 a 36 .

Apesar de a proposta Levine-Athans ter obtido um desempenho melhor para a maior parte das condições iniciais, entende-se que a maior diferença entre os desempenhos, 
Tabela 10 - Comparação entre as funções custo para as propostas Levine-Athans e robusta.

\begin{tabular}{cc}
\hline Con.In. & $J_{L A} / J_{r}$ \\
\hline 1 & 3,31 \\
2 & 3,15 \\
3 & 1,86 \\
4 & 1,84 \\
5 & 1,40 \\
6 & 1,38 \\
7 & 0,61 \\
8 & 0,62 \\
9 & 1,23 \\
\hline
\end{tabular}

\begin{tabular}{cc}
\hline Con.In. & $J_{L A} / J_{r}$ \\
\hline 10 & 1,23 \\
11 & 1,17 \\
12 & 1,20 \\
13 & 1,19 \\
14 & 1,19 \\
15 & 0,69 \\
16 & 0,69 \\
17 & 0,71 \\
18 & 0,71 \\
\hline
\end{tabular}

\begin{tabular}{cc}
\hline Con.In. & $J_{L A} / J_{r}$ \\
\hline 19 & 1,06 \\
20 & 1,06 \\
21 & 0,76 \\
22 & 0,76 \\
23 & 0,77 \\
24 & 0,77 \\
25 & 0,80 \\
26 & 0,80 \\
27 & 0,86 \\
\hline
\end{tabular}

\begin{tabular}{cc}
\hline Con.In. & $J_{L A} / J_{r}$ \\
\hline 28 & 0,86 \\
29 & 0,88 \\
30 & 0,88 \\
31 & 0,99 \\
32 & 0,98 \\
33 & 0,97 \\
34 & 0,97 \\
35 & 0,95 \\
36 & 0,95 \\
\hline
\end{tabular}

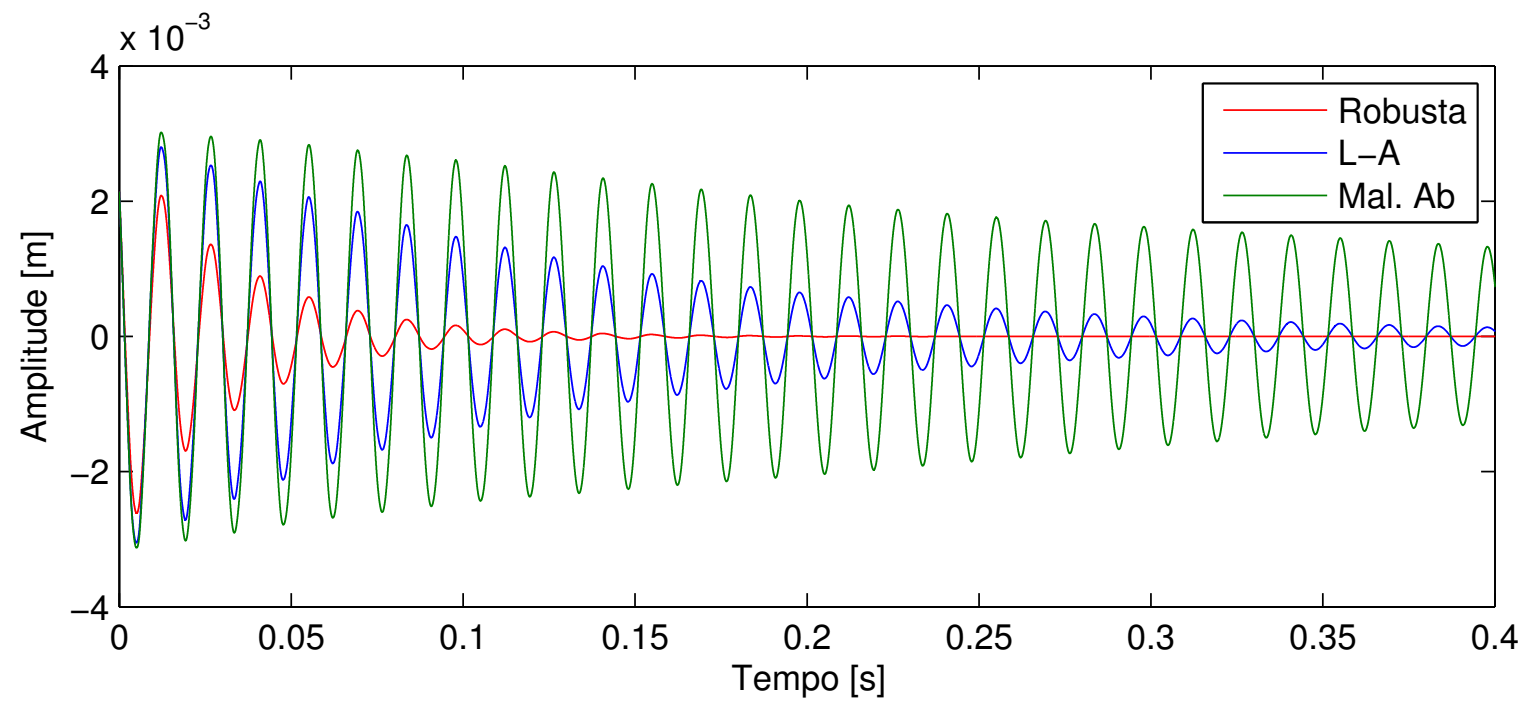

Figura 29 - Comparação entre as respostas temporais para a condição inicial 1 considerando medida de deslocamento na posição $\boldsymbol{\alpha}_{r}$.

de 63,49\% favorável a proposta Levine-Athans e de 231,36\% a favor da proposta robusta, é um fator decisivo para a comparação, pois para pequenas diferenças as respostas de ambos sistemas tendem a ser semelhantes. Não obstante, para alguns casos em que a proposta Levine-Athans supera a robusta a diferença entre os critérios de desempenho é praticamente desprezível. Comparações utilizando as condições mais favoráveis para cada uma das propostas estão indicadas da Figura 29 a Figura 32, em que foram escolhidos pontos de medição de deslocamento que evidenciavam as diferenças desempenho. Nota-se que para a condição inicial 1, a proposta robusta possui desempenho significativamente melhor tanto para a resposta temporal quanto para o esforço de controle. Para a condição inicial 7, observa-se que a proposta Levine-Athans apresenta um desempenho ligeiramente melhor para a resposta temporal com um esforço de controle quase semelhante. A partir desta análise, considera-se que a proposta robusta obteve um desempenho melhor, de modo que a posição $\boldsymbol{\alpha}_{r}$ foi escolhida para o projeto do controle com realimentação de 


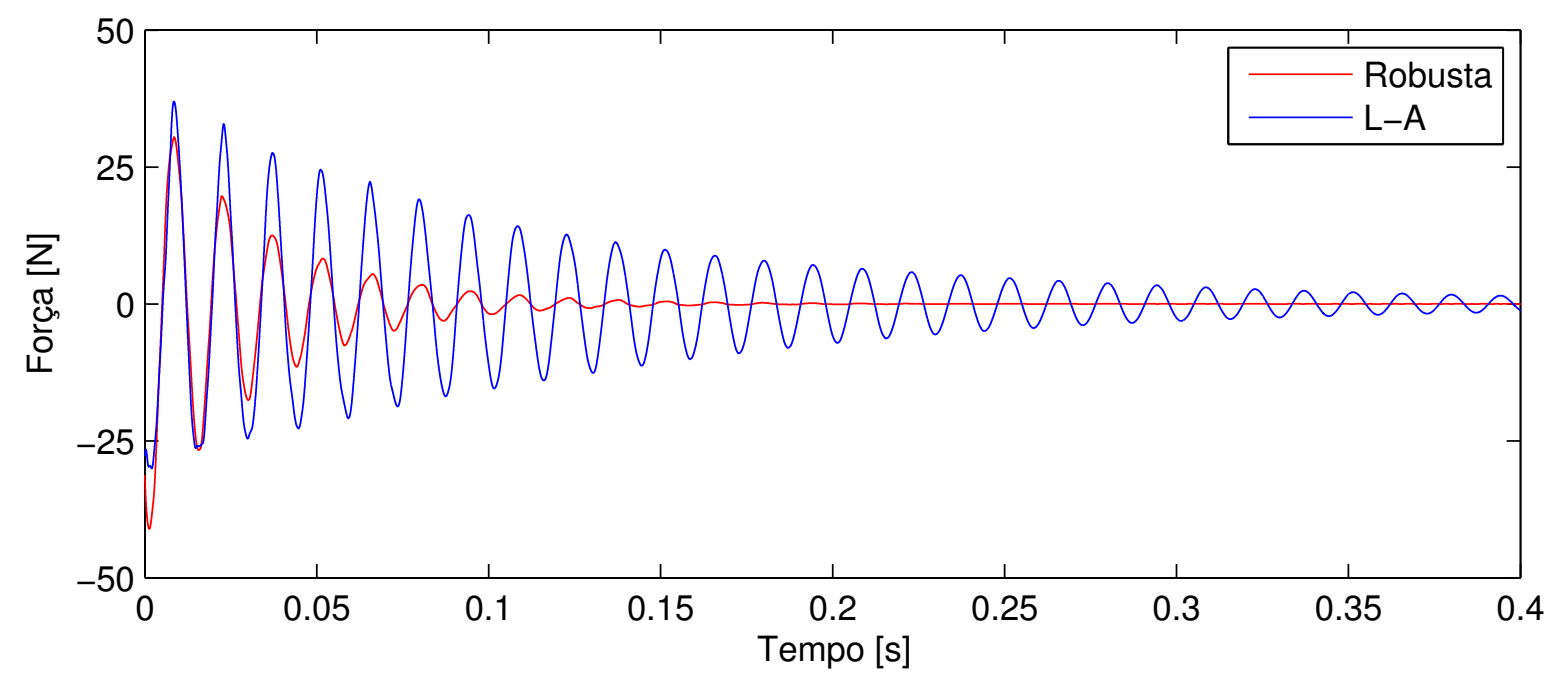

Figura 30 - Comparação entre os esforços de controle para a condição inicial 1.

saída.

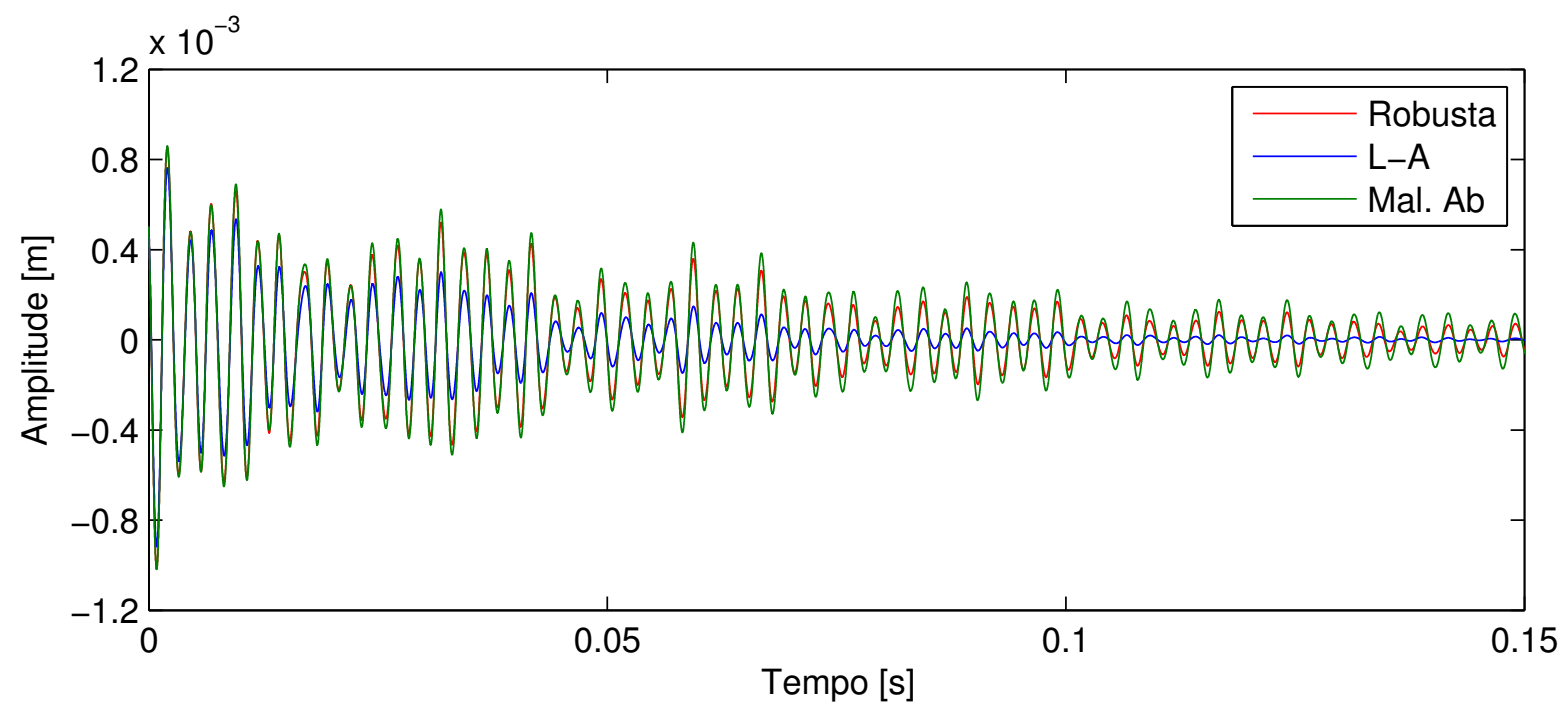

Figura 31 - Comparação entre as respostas temporais para a condição inicial 7 considerando medida de deslocamento na posição $\boldsymbol{\alpha}_{L A}$.

Assim como no caso da viga engastada-livre, analisou-se inicialmente o caso em que os ganhos associados ao deslocamento do controle LQR são anulados. Comparando as duas leis de controle utilizando a condição inicial que maximiza a razão entre as funções custo, observou-se uma diferença quase desprezível, mas um pouco maior que para a viga engastada-livre, de 1,01\%. Estes resultados indicam que, pelo menos para uma avaliação de desempenho a partir das métricas consideradas neste trabalho, a realimentação de deslocamento pode ser desconsiderada.

Portanto, para o projeto do controle com realimentação de saída foram considerados somente sensores com realimentação de velocidade. De início, já foram observadas 


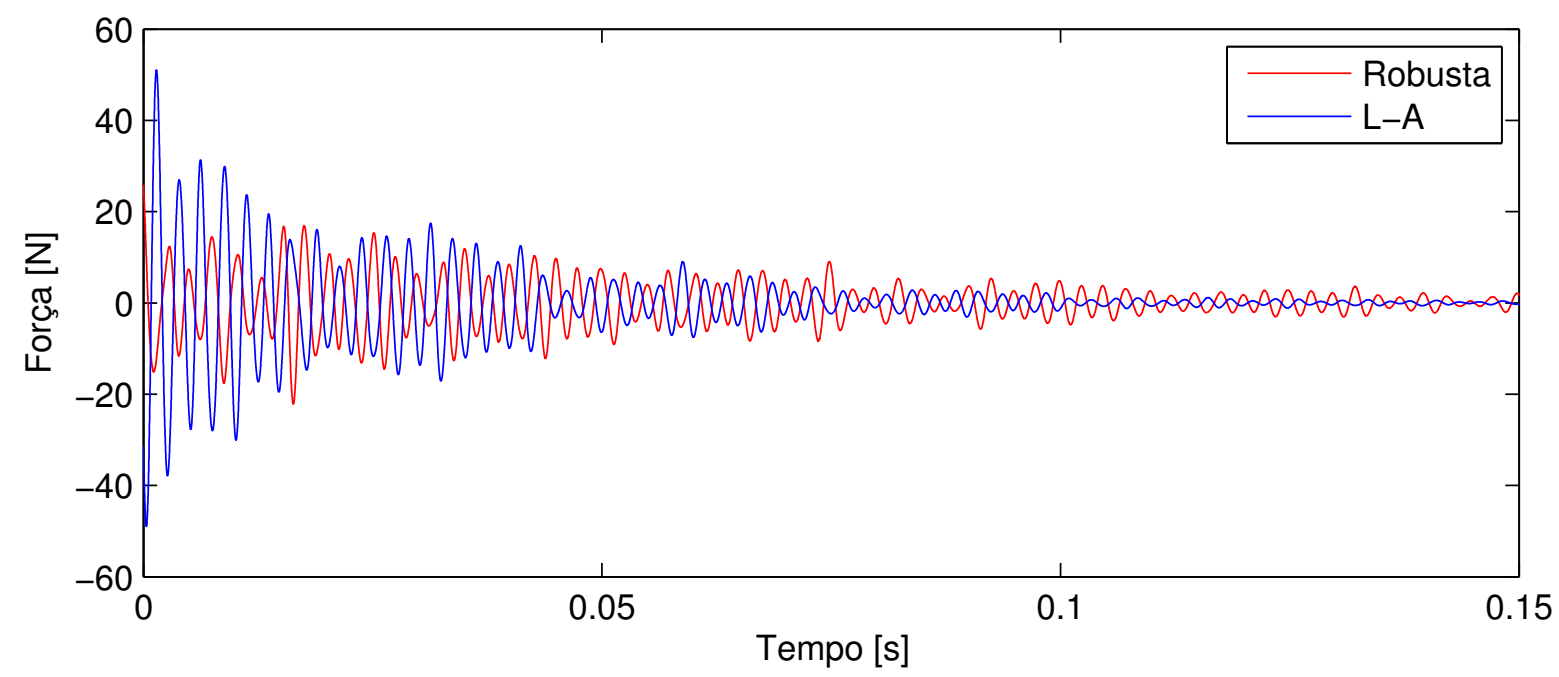

Figura 32 - Comparação entre os esforços de controle para a condição inicial 7.

algumas características diferentes em relação ao exemplo da viga. Considerando somente um sensor colocalizado foi possível obter uma diferença pequena para a função custo do LQR, de somente 7,31\% considerando a pior condição inicial. Utilizando dois sensores não foi possível melhorar tanto o desempenho quanto no caso da viga, já que a máxima diferença obtida para o LQR foi de 4,05\%. Portanto, optou-se por calcular os parâmetros ótimos para diferentes quantidades de sensores e os resultados obtidos estão indicados na Tabela 11. Em todos os casos foi utilizada a abordagem minmax.

Tabela 11 - Parâmetros ótimos e comparação com o LQR para a placa simplesmente apoiada variando o número de sensores.

\begin{tabular}{|c|c|c|c|}
\hline Sensores & Ganhos & Posições $(\mathrm{mm})$ & Máxima diferença para LQR \\
\hline 1 & $k=24,87$ & $\boldsymbol{\xi}=[211,6 \quad 162,9]$ & $7,31 \%$ \\
\hline \multirow{2}{*}{2} & $k_{1}=21,35$ & $\boldsymbol{\xi}_{1}=[224,2 \quad 170,2$ & \multirow{2}{*}{$4,05 \%$} \\
\hline & $k_{2}=8,46$ & $\boldsymbol{\xi}_{2}=[166,1 \quad 118,2$ & \\
\hline \multirow{3}{*}{3} & $k_{1}=10,87$ & $\boldsymbol{\xi}_{1}=[261,9123,7$ & \multirow{3}{*}{$1,91 \%$} \\
\hline & $k_{2}=13,00$ & $\boldsymbol{\xi}_{2}=[160,0 \quad 137,0$ & \\
\hline & $k_{3}=13,68$ & $\boldsymbol{\xi}_{3}=[223,8 \quad 197,0$ & \\
\hline \multirow{4}{*}{4} & $k_{1}=-4,49$ & $\boldsymbol{\xi}_{1}=[434,9 \quad 164,3$ & \multirow{4}{*}{$1,69 \%$} \\
\hline & $k_{2}=6,89$ & $\boldsymbol{\xi}_{2}=[227,954,8]$ & \\
\hline & $k_{3}=24,41$ & $\boldsymbol{\xi}_{3}=[201,4 \quad 170,0$ & \\
\hline & $k_{4}=5,81$ & $\boldsymbol{\xi}_{4}=[310,4164,3$ & \\
\hline \multirow{5}{*}{5} & $k_{1}=20,55$ & $\boldsymbol{\xi}_{1}=\left[\begin{array}{ll}191,6 & 169,3\end{array}\right.$ & \multirow{5}{*}{$1,18 \%$} \\
\hline & $k_{2}=9,36$ & $\boldsymbol{\xi}_{2}=[271,0 \quad 164,9$ & \\
\hline & $k_{3}=-3,65$ & $\boldsymbol{\xi}_{3}=[457,2 \quad 150,5$ & \\
\hline & $k_{4}=-17,17$ & $\boldsymbol{\xi}_{4}=[517,1 \quad 395,9$ & \\
\hline & $k_{5}=20,43$ & $\boldsymbol{\xi}_{5}=[223,3 \quad 15,8]$ & \\
\hline
\end{tabular}

Os valores obtidos para a máxima diferença entre as funções custo no caso dos sensores não colocalizados foram maiores para a placa do que para a viga. No entanto, a 
partir de simulações no tempo das piores condições iniciais e da resposta em frequência, notou-se que a diferença obtida ainda pode ser considerada como praticamente desprezível. Como exemplo, são comparados da Figura 33 a Figura 35, a resposta no tempo, o esforço de controle e o diagrama de Bode para o LQR e o controle com realimentação de saída utilizando dois sensores. Para a resposta em frequência as maiores reduções de amplitude foram para o primeiro, segundo, oitavo, terceiro e quarto modo, de $22,8 d B, 12,5 d B$, $8,3 d B, 8,1 d B$ e $6,3 d B$, respectivamente. Assim como para o exemplo da viga engastadalivre, nota-se que com um número muito menor de sensores obteve-se um desempenho equivalente ao do regulador linear quadrático.

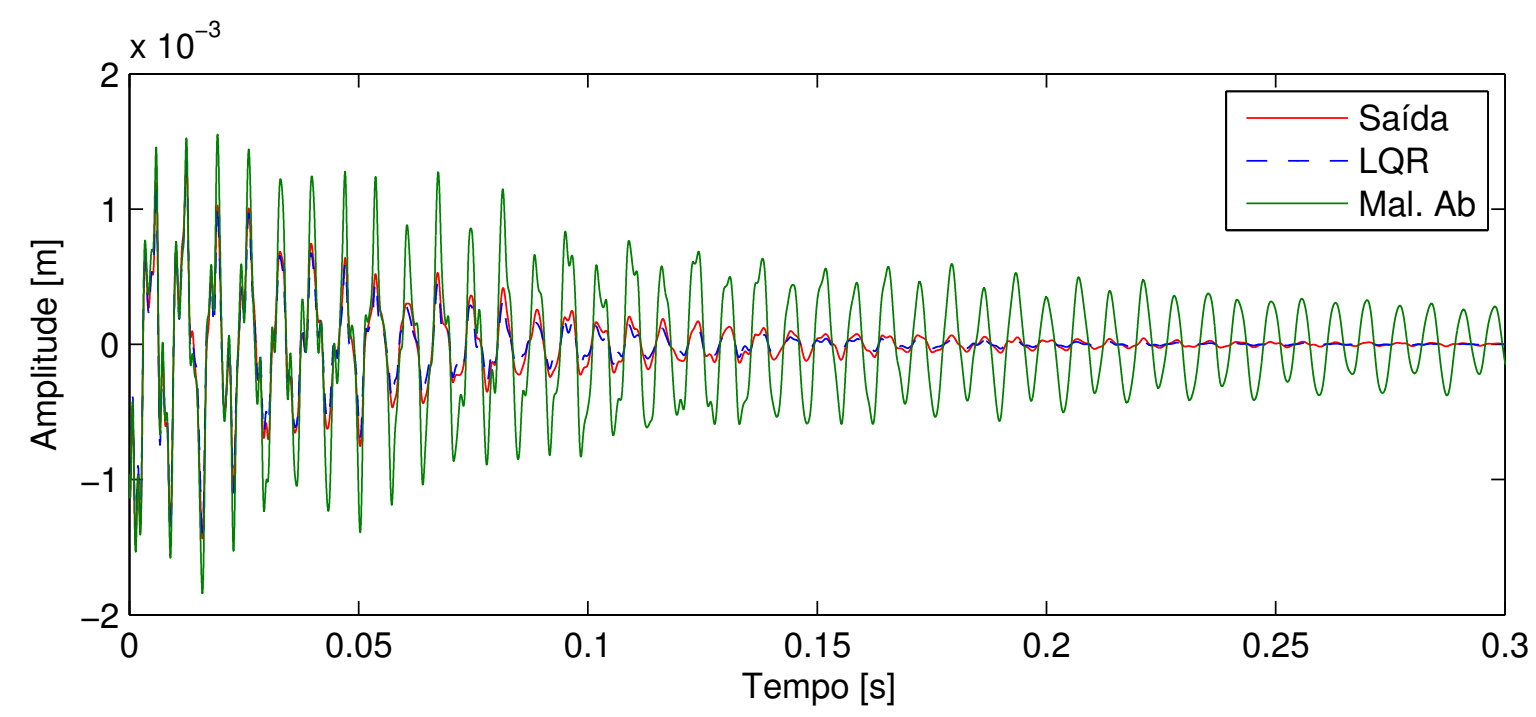

Figura 33 - Comparação entre as respostas temporais de um ponto na posição $\boldsymbol{\alpha}_{r}$ considerando a pior condição inicial do controle com realimentação de saída.

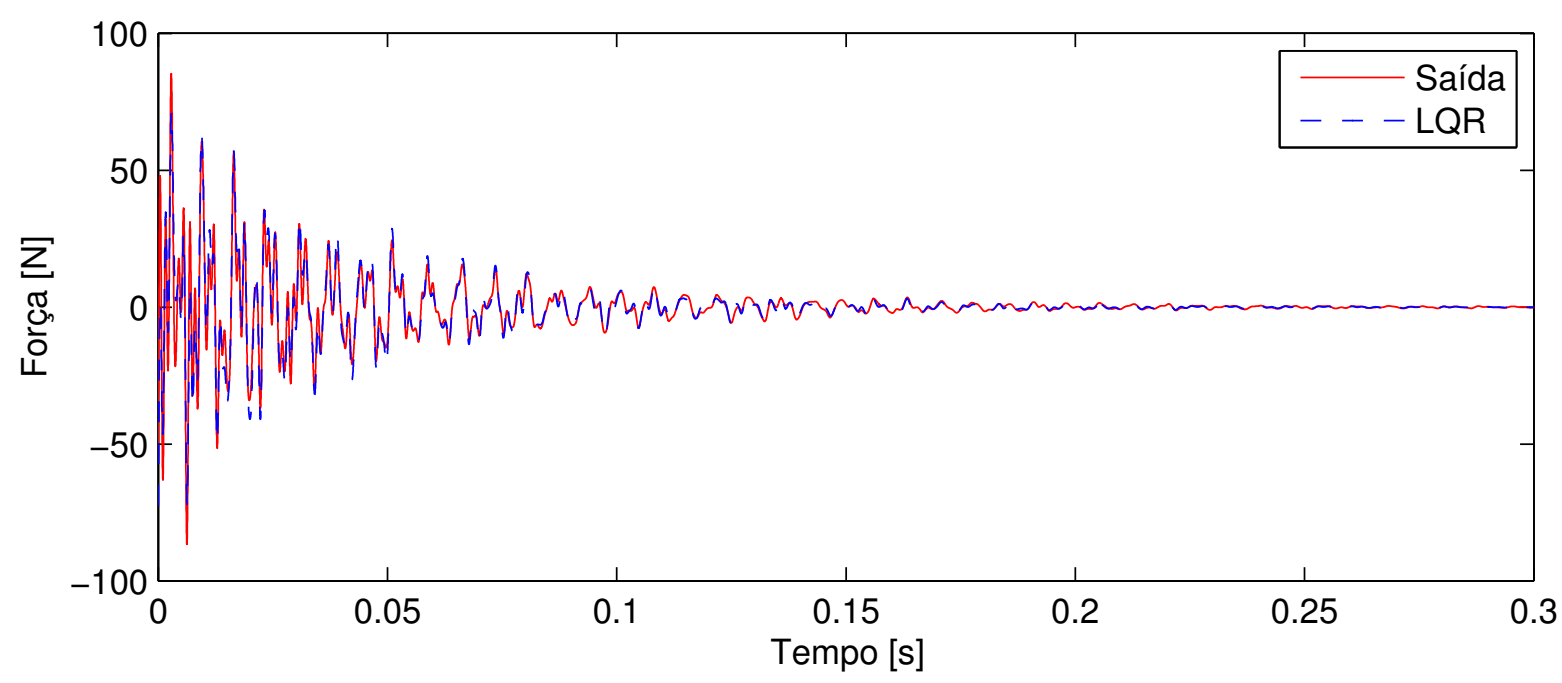

Figura 34 - Comparação entre os esforços de controle considerando a pior condição inicial do controle com realimentação de saída. 


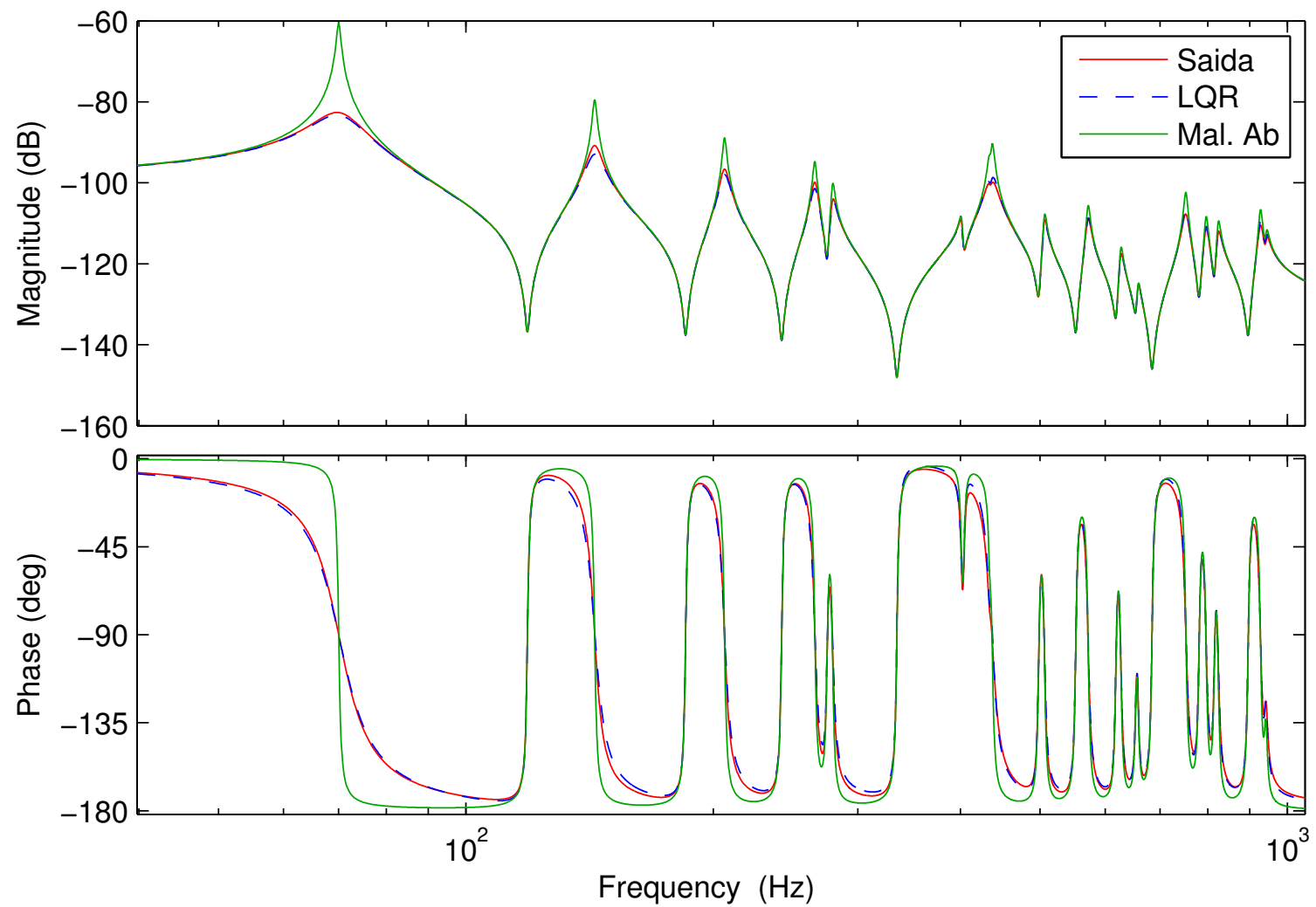

Figura 35 - Comparação entre as respostas em frequência considerando a entrada e a saída na posição $\boldsymbol{\alpha}_{r}$.

\subsubsection{Variação de Parâmetros}

Assim como para a viga engastada-livre, também foi analisado o desempenho e estabilidade do sistema de controle projetado para a placa a partir da variação de parâmetros. Neste caso, qualquer uma das configurações indicadas na Tabela 11 poderia ser utilizada, e optou-se novamente por um caso com dois sensores. A partir do cálculo dos autovalores para diferentes combinações de ganhos dos sensores, verificou-se que ambos possuíam uma margem de ganho considerável. Na Figura 36 é indicada a alteração no critério de desempenho a partir de uma variação de \pm 8 em relação ao valor ótimo para cada um dos ganhos. Nota-se que na vizinhança dos pontos ótimos ainda é possível variar os ganhos sem acometer significativamente o desempenho. Avaliou-se também o limite de variação dos ganhos para o sistema permanecer estável, sendo que para o sensor mais distante do atuador foi necessário aumentar o ganho para aproximadamente 90 parece observar instabilidade mantendo o ganho do primeiro sensor. Para o sensor mais próximo verificou-se que mesmo para variações de algumas ordens de grandeza o sistema em malha fechada ainda permanecia estável.

No caso das posições das sensores, a estabilidade do sistema também foi avaliada 


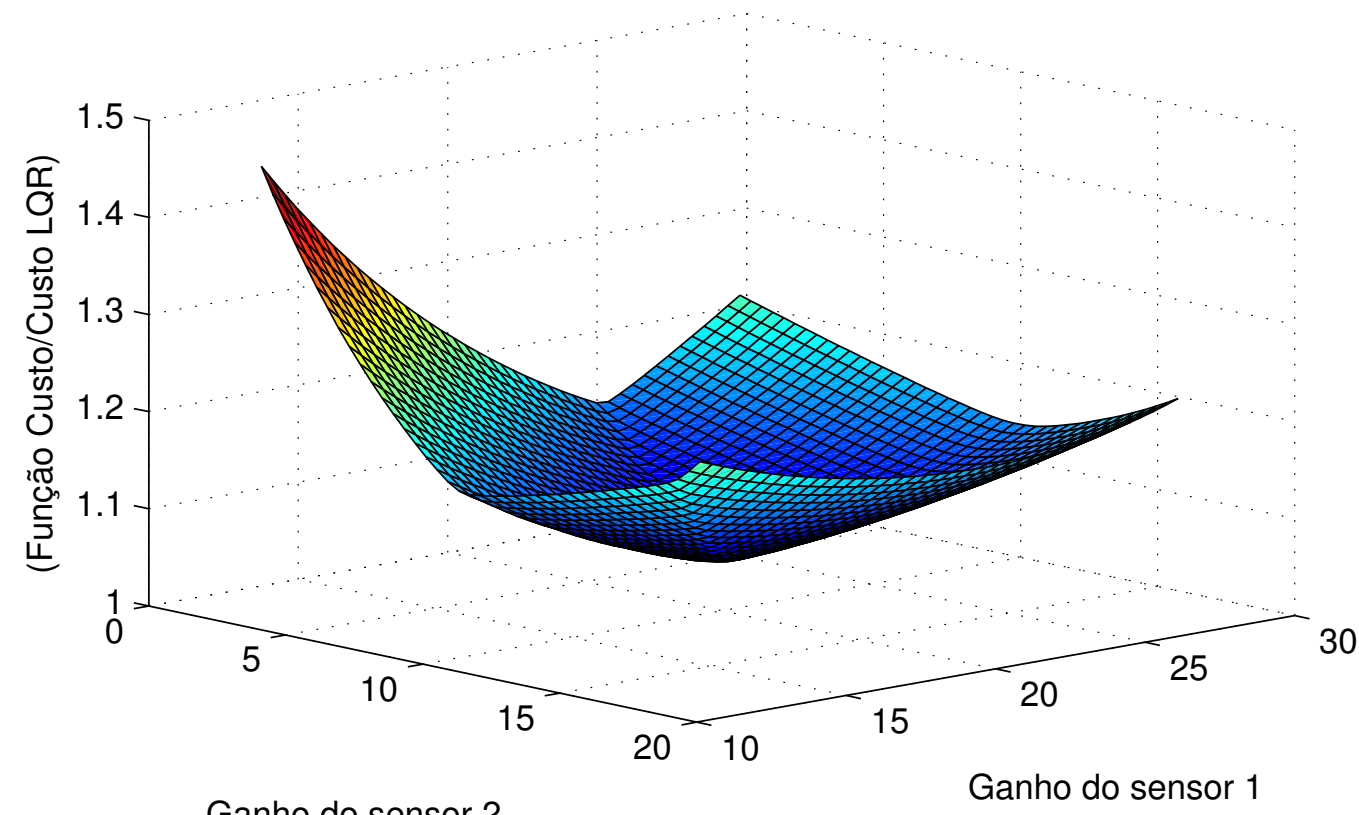

Figura 36 - Máximo valor da razão entre as funções custo do controle de saída e LQR quando os ganhos dos sensores são variados.

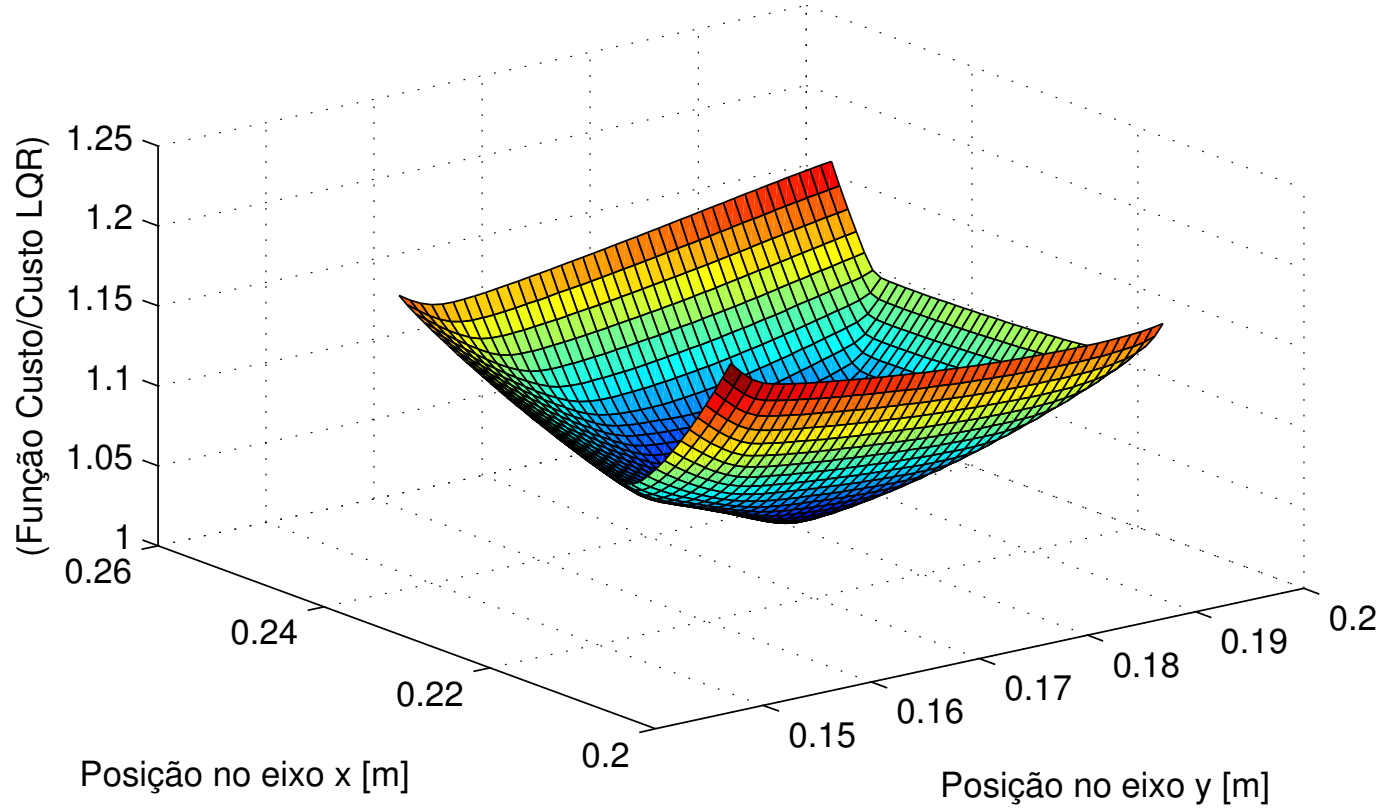

Figura 37 - Máximo valor da razão entre as funções custo do controle de saída e LQR quando a posição do sensor 1 é variada.

a partir do cálculo dos autovalores para diferentes configurações. Como a estrutura é bidimensional, a função custo neste caso é função de quatro variáveis. Desse modo, a superfície do critério de performance foi gerada para cada um dos sensores, e os resultados estão indicados na Figura 37 e Figura 38. Para cada um dos eixos, a posição dos sensores 
foi variada em $\pm 20 \mathrm{~mm}$, e ainda assim verificou-se que o desempenho do sistema foi pouco afetado.

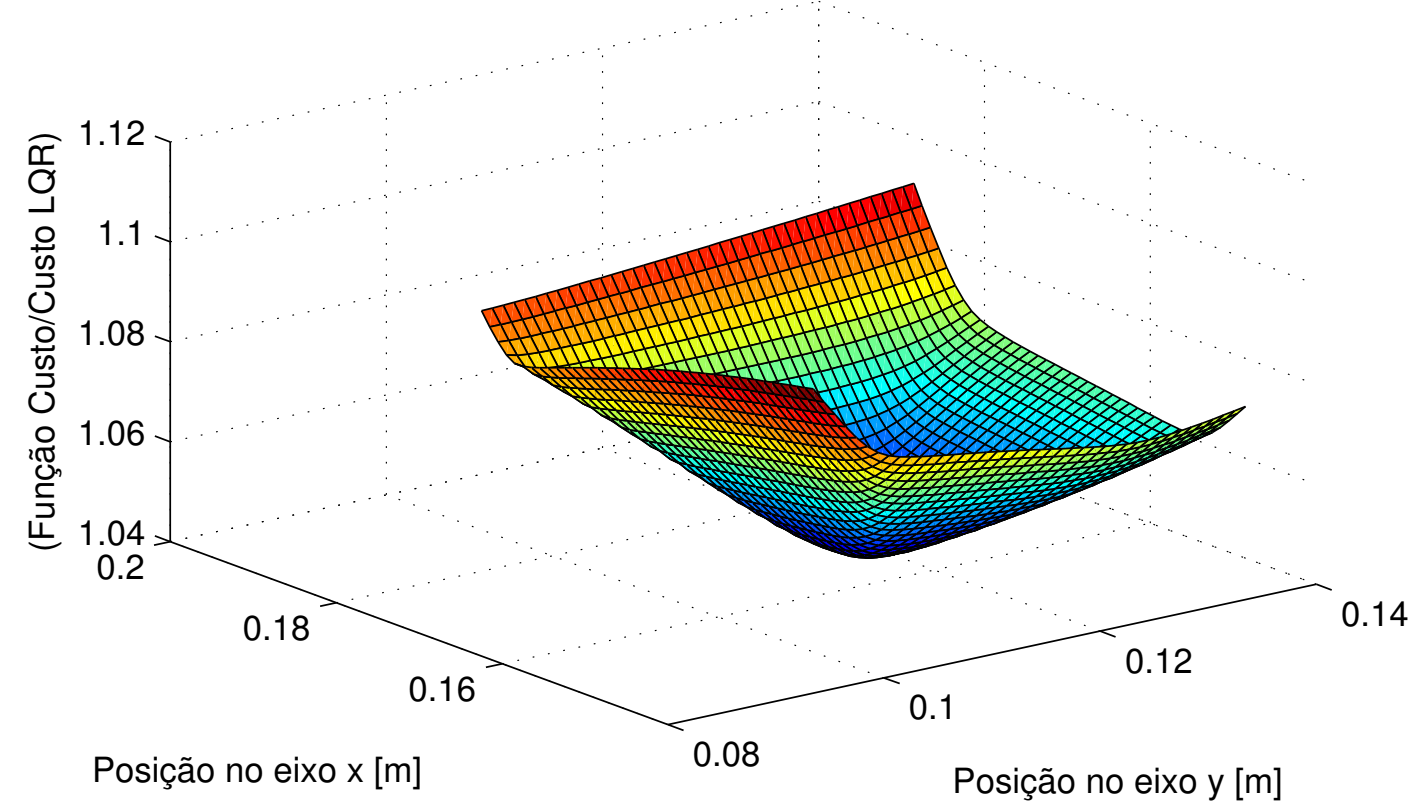

Figura 38 - Máximo valor da razão entre as funções custo do controle de saída e LQR quando a posição do sensor 2 é variada.

Estas análises avaliam a estabilidade do sistema para um alteração individual dos parâmetros. Para avaliar o caso de variações combinadas, considerou-se que os parâmetros ótimos poderiam ser variados em uma faixa de $\pm i \%$, em que $i \in\{1,2, \ldots\}$. Incrementando os valores de $i$ neste conjunto, notou-se que somente a partir de uma variação de $19 \%$ nos parâmetros era observada uma instabilidade no sistema. Este resultado indica que além de um desempenho muito próximo do LQR, o controle com realimentação de saída ainda pode exibir propriedades consideráveis de robustez. Assim como para a viga, ainda analisou-se em particular um caso em que foi considerada uma variação de $+5 \%$ em todos os parâmetros ótimos, de modo a obter as posições $\boldsymbol{\xi}_{1}=\left[\begin{array}{ll}235,5 & 178,7\end{array}\right]$, $\boldsymbol{\xi}_{2}=[174,5124,1]$ e o ganho $\boldsymbol{k}=[22,428,89]$. A condição inicial que implicava na maior diferença entre os critérios de desempenho deste caso e o ótimo foi determinada e comparações de desempenho entre ambos os casos são ilustradas na Figura 39 e na Figura 40. Em todas as figuras a seguir considerou-se medição de deslocamento na posição do atuador. Nota-se que apesar de a resposta temporal ser equivalente, obteve-se um acréscimo relevante no esforço de controle para o caso de variação de 5\%. Ainda assim, este é o pior caso e espera-se que o comportamento de ambos sistemas seja mais próximo para outras excitações.

Por último, avaliou-se o novamente o fenômeno de spillover. Inicialmente, considerouse o ponto ótimo do sistema com dois sensores (linha 3 da Tabela 11) e uma ampliação na faixa de frequências para até $2 k H z$, o que implicou na discretização do modelo em até 40 

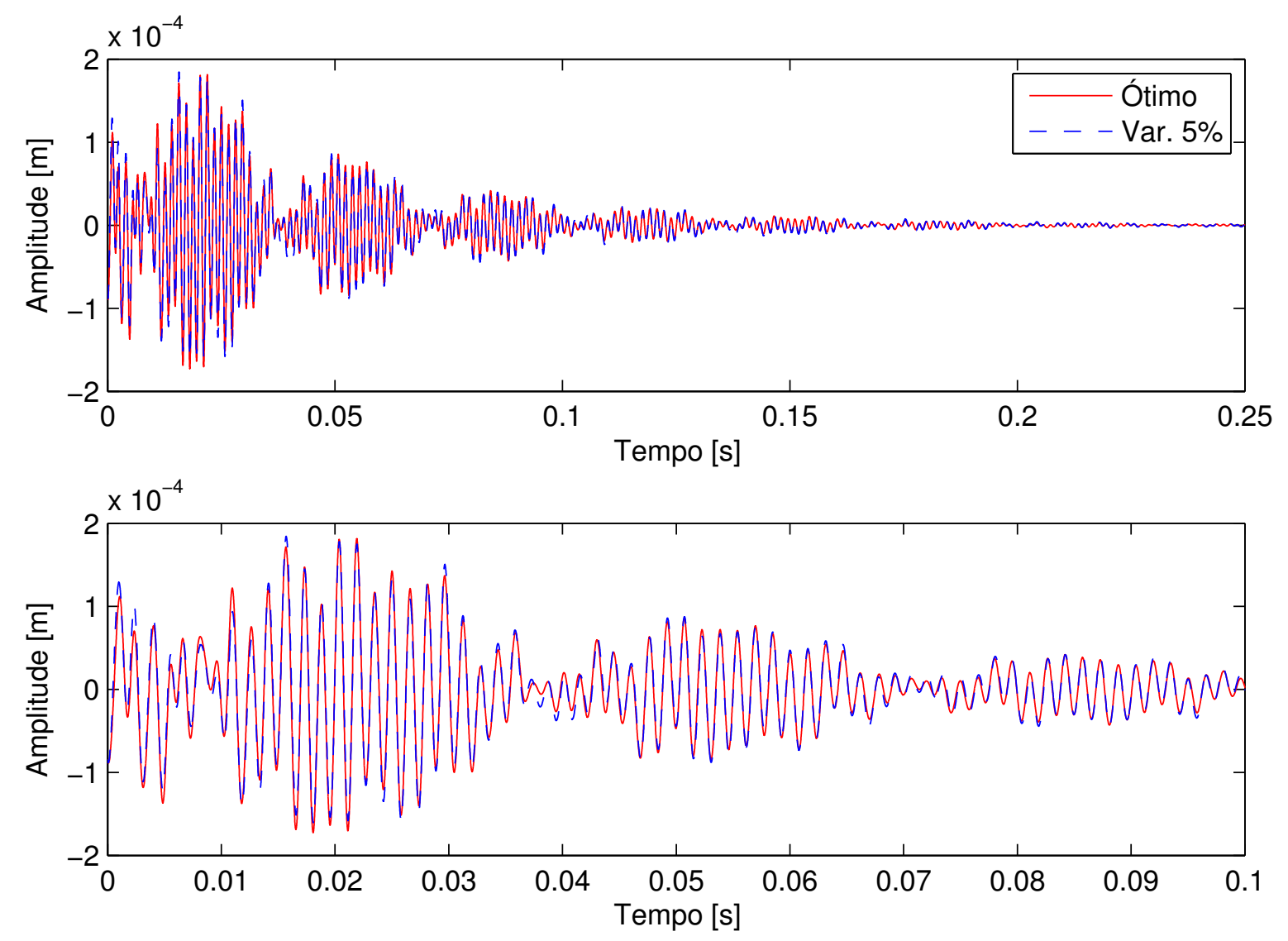

Figura 39 - Comparação entre as respostas temporais dos sistemas com parâmetros ótimos e com erro de $5 \%$.

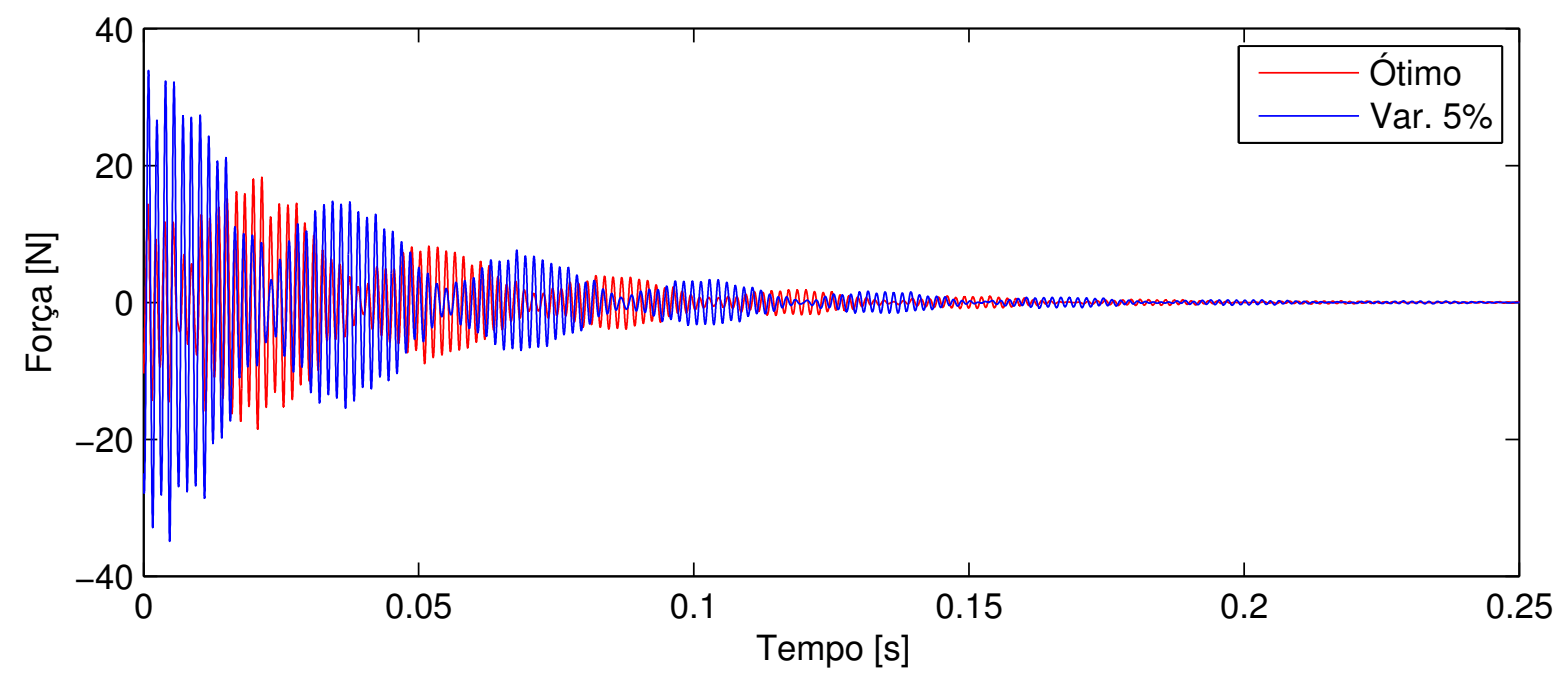

Figura 40 - Comparação entre os esforços de controle dos sistemas com parâmetros ótimos e com erro de $5 \%$.

modos. O número de modos foi incrementado de 18 a 40, e em todos os casos verificou-se que o sistema em malha fechada permanecia estável para realimentação de velocidade dos dois sensores. Do mesmo modo que para a viga, o desempenho do sistema projetado 
para 18 modos se deteriorava conforme a inclusão de modos no modelo. Como exemplo, para o sistema com 30 modos a máxima diferença entre o controle com realimentação de saída e o LQR foi de 12,66\%, enquanto para 40 modos essa diferença atingiu 30, 30\%. No entanto, as condições iniciais que implicavam em diferenças maiores em relação ao LQR correspondiam aos modos de alta frequência, de modo que o desempenho do sistema para baixa frequência não foi alterado com a inclusão de modos no modelo. Da Figura 41 a Figura 45 foram comparadas as respostas temporais e esforços de controle entre o LQR o controle com realimentação de saída utilizando o ponto ótimo do sistema com 18 modos, identificado como "O18", para condições iniciais dadas por modos de alta e baixa frequência. Na Figura 45 também foi realizada uma comparação para a faixa de $2 \mathrm{kHz}$ através da resposta em frequência.

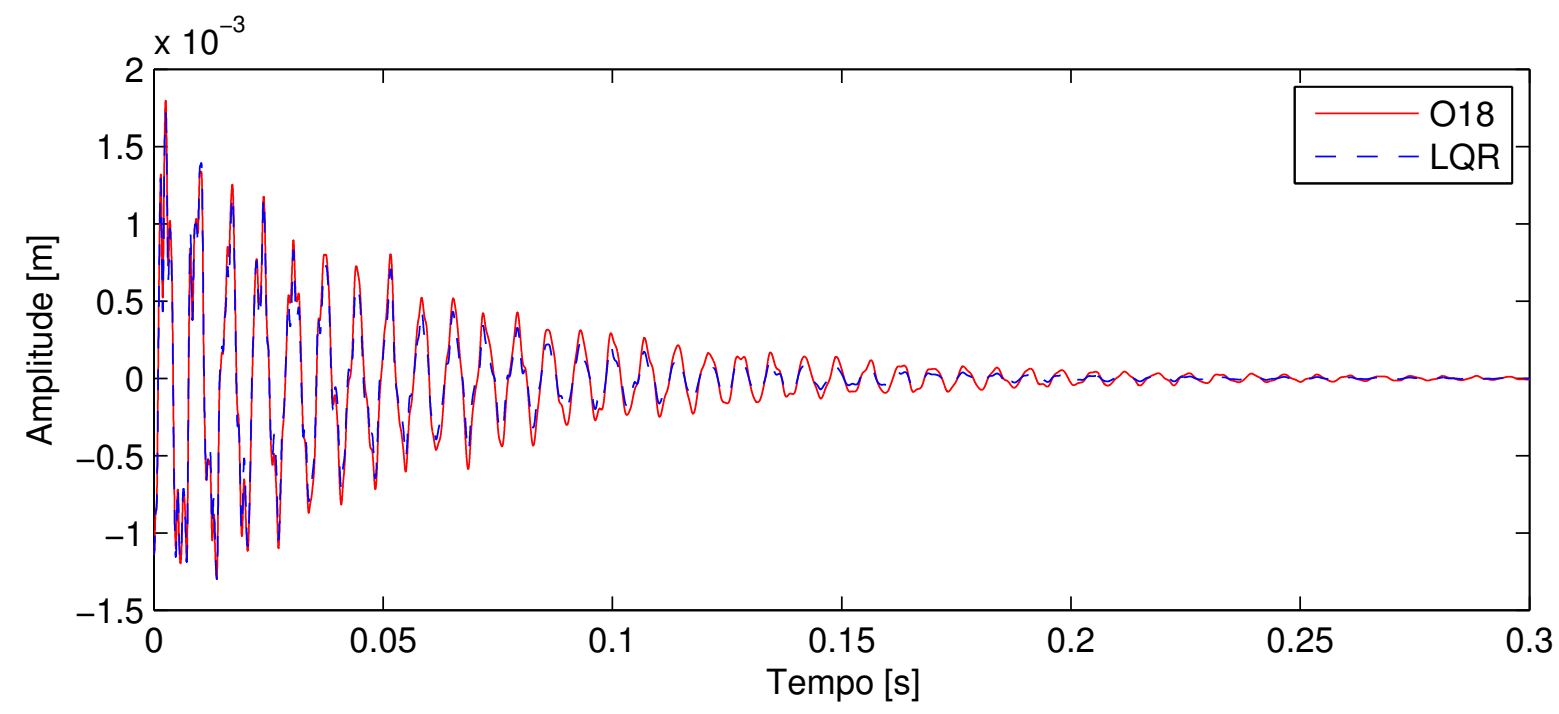

Figura 41 - Comparação das respostas temporais entre LQR e controle com realimentação de saída para uma condição inicial dada aproximadamente por uma velocidade inicial no $2^{\circ}$ modo. 


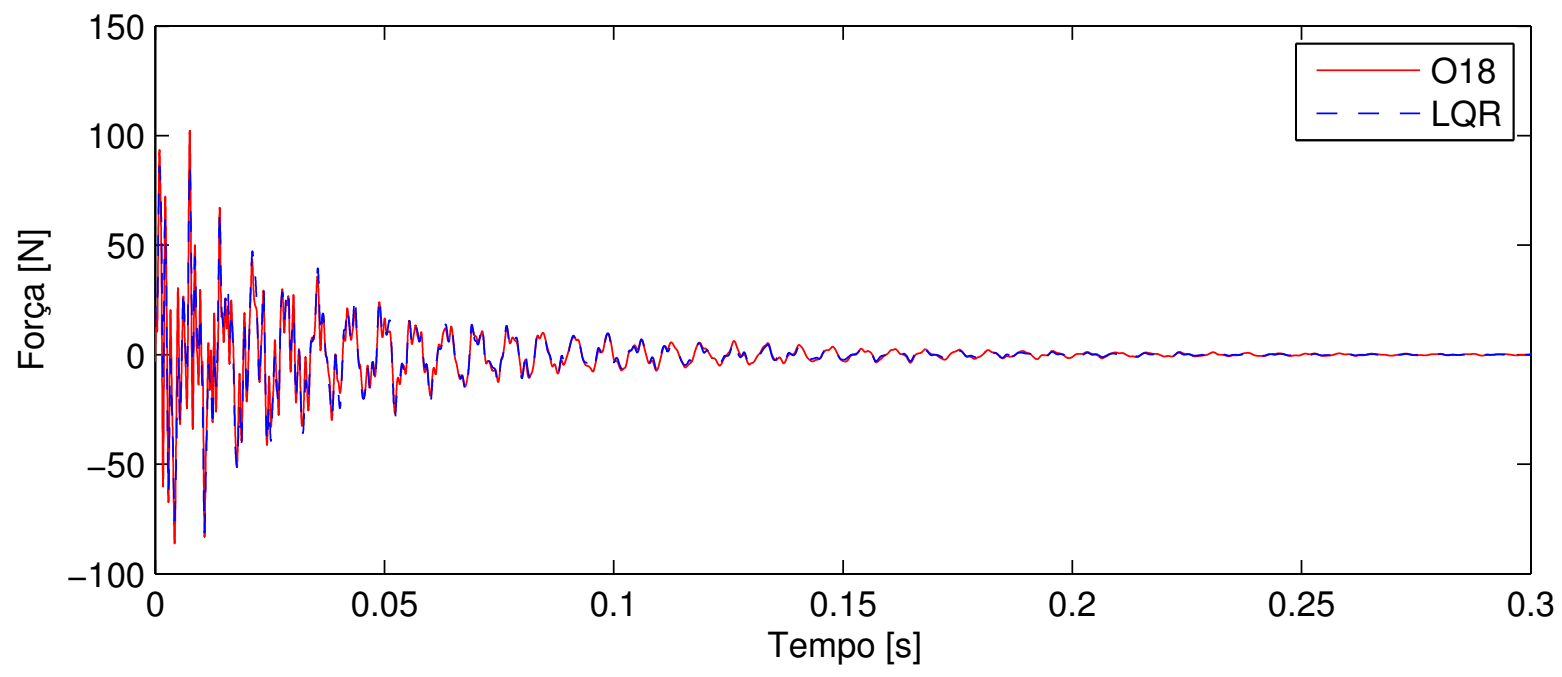

Figura 42 - Comparação dos esforços de controle entre LQR e controle com realimentação de saída para uma condição inicial dada aproximadamente por uma velocidade inicial no $2^{\circ}$ modo.
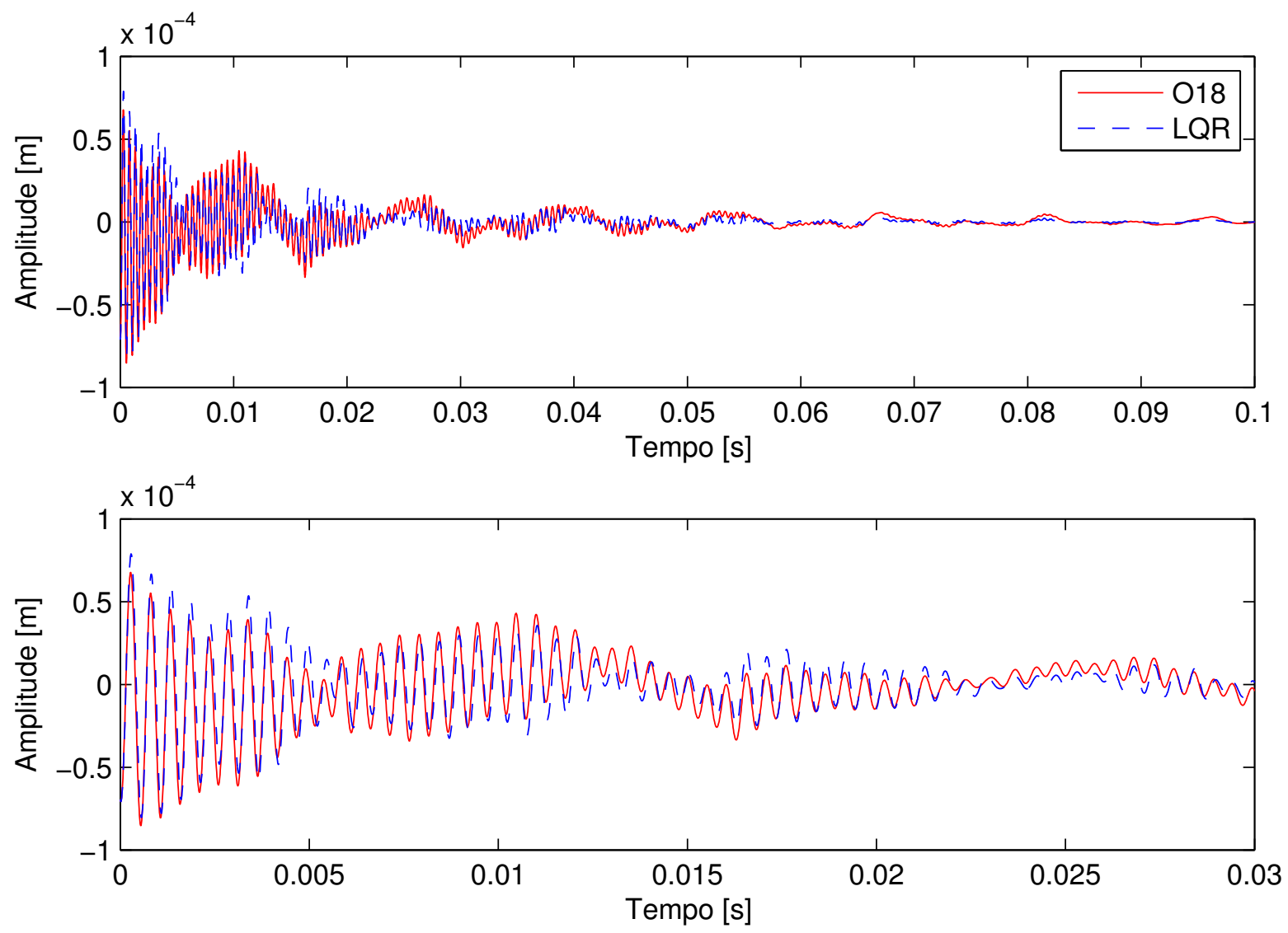

Figura 43 - Comparação das respostas temporais entre LQR e controle com realimentação de saída para uma condição inicial dada aproximadamente por uma velocidade inicial no $40^{\circ}$ modo. 


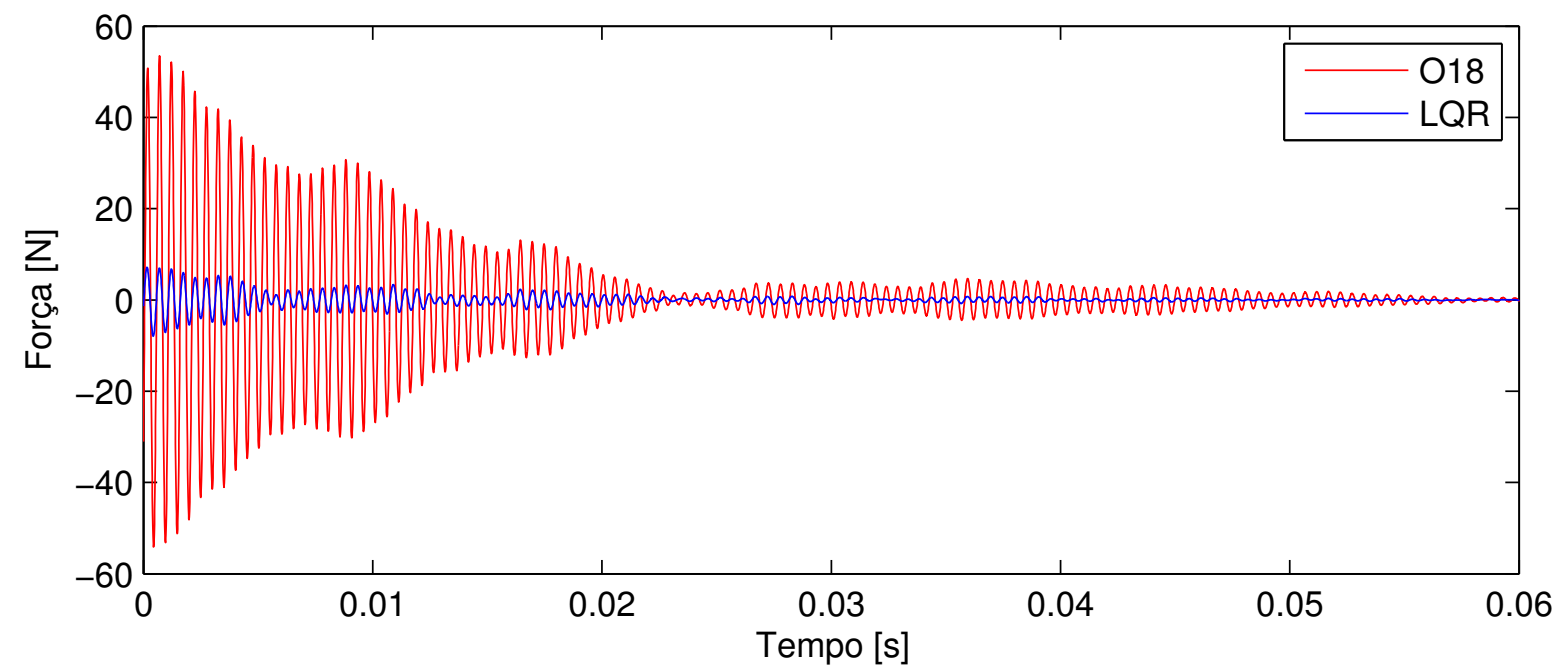

Figura 44 - Comparação dos esforços de controle entre LQR e controle com realimentação de saída para uma condição inicial dada aproximadamente por uma velocidade inicial no $40^{\circ}$ modo.

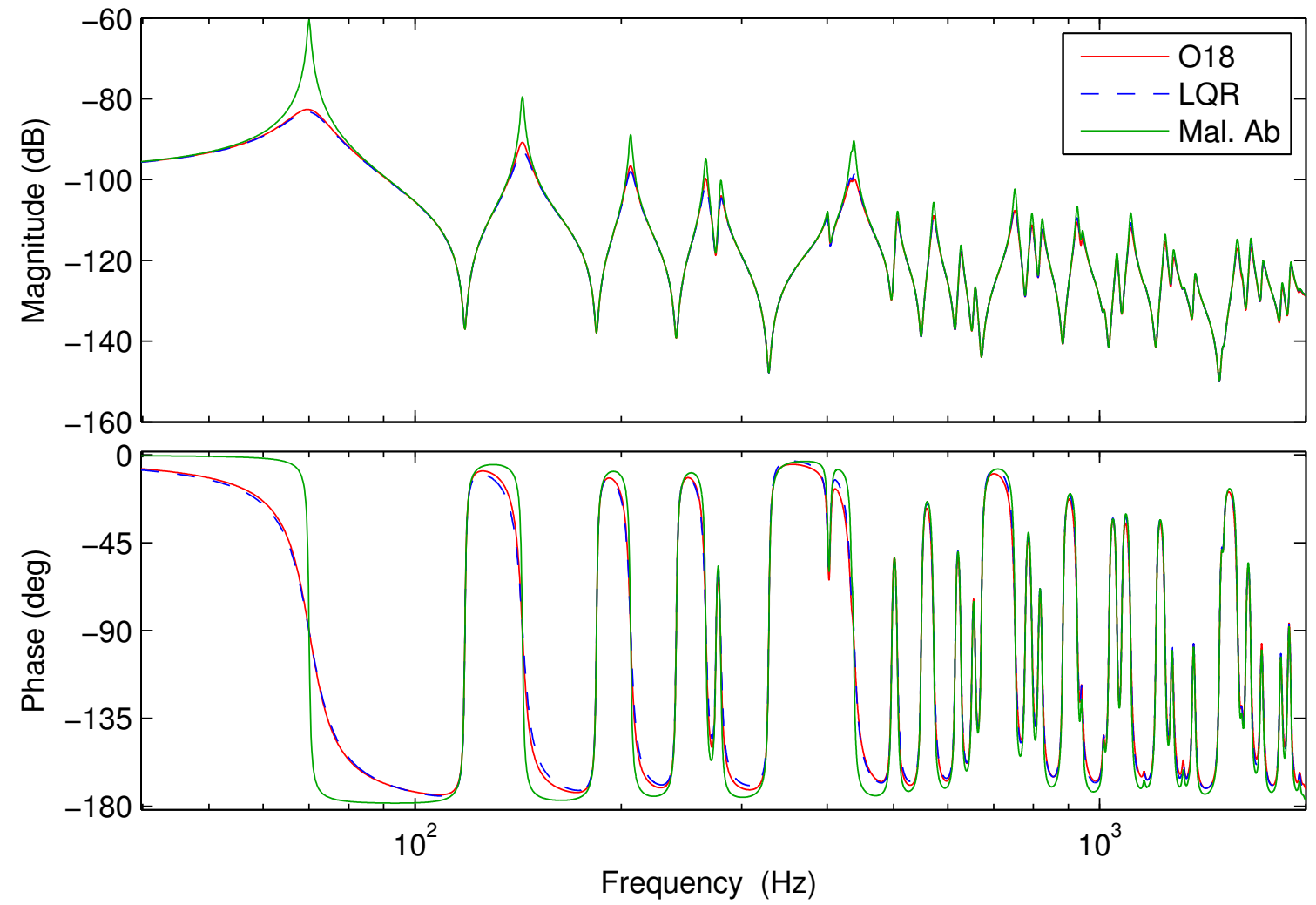

Figura 45 - Comparação entre LQR e controle com realimentação de saída em $2 \mathrm{kHz}$ através do diagrama de Bode. 



\section{Considerações Finais}

\subsection{Conclusões}

Neste trabalho, estudou-se o problema de controle de vibrações em estruturas flexíveis considerando a otimização de um critério de desempenho quadrático com restrição de realimentação de saída, cujas variáveis de otimização eram dadas pelas posições dos sensores, atuadores e ganho de controle. Determinaram-se as equações que representavam as condições necessárias de otimalidade e foram identificados os principais problemas para obtenção das soluções.

O problema de existência de parâmetros ótimos que garantissem a estabilidade foi resolvido a partir da especificação de uma forma modal para o sistema e utilizando funções de controle de Lyapunov, o que adicionalmente proporcionou o resultado inédito, segundo o conhecimento do autor e pesquisas em bases de dados, de que o controle colocalizado é solução do problema do regulador linear quadrático.

Para a dependência da solução com relação à condição inicial foram propostas duas abordagens. Na primeira, que foi utilizada para a determinação das posições dos atuadores e determinação do ganho de realimentação de estados, especificava-se um conjunto provável de condições iniciais para o sistema e otimizava-se para o pior caso. Na outra, utilizada para determinar as posições dos sensores e ganho de realimentação de saída, introduzia-se uma modificação na função custo, de modo a tentar aproximar o desempenho do controle com realimentação de saída do regulador linear quadrático para a condição inicial que maximizava a razão entre os critérios de desempenho.

A metodologia proposta foi aplicada para dois exemplos de dinâmica estrutural: uma viga engastada-livre e uma placa simplesmente apoiada em todos os lados. Em ambos exemplos, verificou-se que mesmo para uma razão pequena entre o número de sensores pelo número de estados era possível obter um desempenho equivalente ao controle LQR. Além disso, mostrou-se que as abordagens propostas para lidar com a dependência em relação à condição inicial proporcionavam resultados melhores que a proposta usual de assumir que a condição inicial é uma variável aleatória uniformemente distribuída em uma esfera.

Por fim, realizou-se uma análise de sensibilidade com relação às variáveis de otimização para ambos exemplos. Em ambos os casos observou-se que mesmo para uma variação significativa nos parâmetros o sistema em malha fechada permanecia estável. Para uma pequena variação nos parâmetros mostrou-se que o desempenho do sistema alterava-se ligeiramente, mostrando baixa sensibilidade. O fenômeno de spillover também 
foi estudado, e mesmo para uma inclusão de um número considerável de modos ainda assim obteve-se estabilidade.

Algumas conclusões obtidas são decorrentes da aplicação em exemplos, de modo que não é possível garantir o mesmo comportamento para qualquer caso. No entanto, os resultados obtidos indicam que o principal problema para o projeto do controle com realimentação de saída é determinar um controle LQR com desempenho satisfatório, já que mesmo com um número reduzido de sensores foi possível obter um desempenho equivalente. Portanto, entende-se que a metodologia proposta é uma boa alternativa para as técnicas de controle LQR e LQG. Ainda assim, julga-se que estudos posteriores avaliando o caso de diferentes metodologias para o projeto do controle LQR e considerando estruturas mais complexas são necessários para verificar a eficácia do método proposto.

A divulgação científica dos resultados apresentados nesta dissertação já foi iniciada, e parte dos resultados aqui contidos foram apresentados no 24th ABCM International Congress of Mechanical Engineering, cujo artigo ainda deve ser publicado nos anais do evento. Para divulgação dos resultados que ainda não foram apresentados, planeja-se a escrita e submissão de artigos para outros congressos e periódicos.

\subsection{Principais Contribuições}

- Demonstrar de que o controle colocalizado é solução do problema do regulador linear quadrático;

- Mostrar que há uma dependência significativa dos parâmetros ótimos em relação à condição inicial do sistema dinâmico, mas que a metodologia proposta pode resolver esse problema de maneira eficiente;

- Mostrar que é possível obter um controle com realimentação de saída com desempenho muito próximo de LQR, mesmo utilizando um número reduzido de sensores;

- Mostrar que o controle obtido possui propriedades de robustez em relação às variações de parâmetros, como posições e ganho dos sensores.

\subsection{Trabalhos Futuros}

- Aplicar a metodologia proposta para o caso de sensores e atuadores distribuídos, como pastilhas piezelétricas;

- Realizar um estudo de verificação experimental do método desenvolvido; 
- Avaliar outras metodologias para o projeto do regulador linear quadrático a fim de verificar se sempre é possível obter um desempenho equivalente com o controle com realimentação de saída;

- Aplicar a metodologia para outras estruturas com o objetivo de verificação de sua aplicabilidade;

- Estender o estudo de dependência da eficácia da metodologia com faixa de frequência e número de sensores.

- Avaliar a robustez do sistema em relação às variações de parâmetros da estrutura. 



\section{Referências}

ABDULLAH, M. M. Optimal placement of output feedback controllers on slender civil structures at discrete locations. Engineering Structures, v. 22, n. 8, p. 1042-1047, 2000. Citado na página 32 .

ALIEV, F. A. et al. High-accuracy algorithms to the solution of the optimal output feedback problem for the linear systems. Proceedings of the Romanian Academy Series a-Mathematics Physics Technical Sciences Information Science, v. 13, n. 3, p. 207-214, 2012. Citado na página 28.

ANDERSON, B. D.; MOORE, J. B. Linear optimal control. [S.l.]: Prentice-Hall Englewood Cliffs, NJ, 1971. v. 197. Citado 3 vezes nas páginas 27, 52 e 80.

ARNOLD, W. F.; LAUB, A. J. Generalized eigenproblem algorithms and software for algebraic riccati-equations. Proceedings of the IEEE, v. 72, n. 12, p. 1746-1754, 1984. Citado na página 70.

ARTSTEIN, Z. Stabilization with relaxed controls. Nonlinear Analysis-Theory Methods E6 Applications, v. 7, n. 11, p. 1163-1173, 1983. Citado na página 50.

BELLMAN, R. Introduction to matrix analysis. [S.l.]: SIAM, 1997. Citado 2 vezes nas páginas 27 e 50.

BEN-TAL, A.; GHAOUI, L. E.; NEMIROVSKI, A. Robust optimization. [S.l.]: Princeton University Press, 2009. Citado na página 61.

BROCKETT, R. W.; BYRNES, C. I. Multivariable nyquist criteria, root loci, and pole placement - a geometric viewpoint. IEEE Transactions on Automatic Control, v. 26, n. 1, p. 271-284, 1981. Citado na página 29.

BURKE, J. V. et al. Stabilization via nonsmooth, nonconvex optimization. IEEE Transactions on Automatic Control, v. 51, n. 11, p. 1760-1769, 2006. Citado na página 29.

BURKE, J. V.; LEWIS, A. S.; OVERTON, M. L. A robust gradient sampling algorithm for nonsmooth, nonconvex optimization. Siam Journal on Optimization, v. 15, n. 3, p. 751-779, 2005. Citado na página 29.

CAI, G.-P.; LIM, C. Continuous suboptimal control with partial state feedback. Journal of Vibration and Control, v. 11, n. 4, p. 561-578, 2005. Citado 2 vezes nas páginas 26 e 33 .

CHANDIRAMANI, N. K. Semiactive control of earthquake/wind excited buildings using output feedback. International Conference on Vibration Problems 2015, v. 144, p. 1294-1306, 2016. Citado 2 vezes nas páginas 28 e 31.

CHOI, S. S.; SIRISENA, H. R. Computation of optimal output feedback gains for linear-multivariable systems. IEEE Transactions on Automatic Control, AC19, n. 3, p. 257-258, 1974. Citado 2 vezes nas páginas 27 e 28. 
CHUNG, L. L.; LIN, C. C.; CHU, S. Y. Optimal direct output-feedback of structural control. Journal of Engineering Mechanics-ASCE, v. 119, n. 11, p. 2157-2173, 1993. Citado na página 30.

COURAnT, R.; HILBERT, D. Methods of Mathematical Physics. [S.l.]: Wiley-VHC, 2004. v. 1. Citado na página 37.

CRAIG, R. R.; KURDILA, A. J. Fundamentals of structural dynamics. [S.l.]: John Wiley \& Sons, 2006. Citado 2 vezes nas páginas 65 e 87.

DARIVANDI, N.; MORRIS, K.; KHAJEPOUR, A. An algorithm for LQ optimal actuator location. Smart Materials and Structures, v. 22, n. 3, 2013. Citado 2 vezes nas páginas 28 e 32 .

DEMETRIOU, M. A. A numerical algorithm for the optimal placement of actuators and sensors for flexible structures. In: 2000 American Control Conference (ACC 2000). [S.l.: s.n.], 2000. (Proceedings of the American Control Conference), p. 2290-2294. Citado 2 vezes nas páginas 31 e 34 .

FIALA, J.; KOčVARA, M.; STINGL, M. Penlab: A matlab solver for nonlinear semidefinite optimization. arXiv preprint arXiv:1311.5240, 2013. Citado na página 29.

FREEMAN, R.; KOKOTOVIC, P. V. Robust nonlinear control design: state-space and Lyapunov techniques. [S.l.]: Springer Science \& Business Media, 2008. Citado na página 57.

FRISWELL, M. I.; INMAN, D. J. The relationship between positive position feedback and output feedback controllers. Smart Materials \& Structures, v. 8, n. 3, p. 285-291, 1999. Citado 3 vezes nas páginas 30, 33 e 34.

GHARIB, M.; OMRAN, A.; EL-BAYOUMI, G. Optimal vibration control for structural-acoustic coupling system. Journal of Vibration and Control, v. 19, n. 1, p. 14-29, 2013. Citado 2 vezes nas páginas 28 e 31.

GOH, C. J.; CAUGHEY, T. K. On the stability problem caused by finite actuator dynamics in the collocated control of large space structures. International Journal of Control, v. 41, n. 3, p. 787-802, 1985. Citado na página 30.

GUPTA, V.; SHARMA, M.; THAKUR, N. Optimization criteria for optimal placement of piezoelectric sensors and actuators on a smart structure: A technical review. Journal of Intelligent Material Systems and Structures, v. 21, n. 12, p. 1227-1243, 2010. Citado na página 31.

HANAGUD, S.; OBAL, M. W.; CALISE, A. J. Optimal vibration control by the use of piezoceramic sensors and actuators. Journal of Guidance Control and Dynamics, v. 15, n. 5, p. 1199-1206, 1992. Citado 2 vezes nas páginas 29 e 69.

HOCK, W.; SCHITTKOWSKI, K. A comparative performance evaluation of 27 non-linear programming codes. Computing, v. 30, n. 4, p. 335-358, 1983. Citado na página 63.

HORISBERGER, H.; BELANGER, P. Solution of the optimal constant output feedback problem by conjugate gradients. IEEE Transactions on Automatic Control, v. 19, n. 4, p. 434-435, 1974. Citado na página 27. 
HOUSNER, G. W. et al. Structural control: Past, present, and future. Journal of Engineering Mechanics-ASCE, v. 123, n. 9, p. 897-971, 1997. Citado na página 25.

KALMAN, R. E. Contributions to the theory of optimal control. Bol. Soc. Mat. Mexicana, v. 5, n. 2, p. 102-119, 1960. Citado na página 51.

KALMAN, R. E. When is a linear control system optimal? Journal of Basic Engineering, v. 86, n. 1, p. 51-60, 1964. Citado na página 52.

KATO, T. Perturbation theory for linear operators. [S.l.]: Springer Science \& Business Media, 2013. v. 132. Citado na página 52.

KUMAR, K. R.; NARAYANAN, S. Active vibration control of beams with optimal placement of piezoelectric sensor/actuator pairs. Smart Materials \& Structures, v. 17, n. 5, p. 15, 2008. Citado na página 32.

LAUB, A. J. Matrix analysis for scientists and engineers. [S.l.]: Siam, 2005. Citado na página 59.

LEIBFRITZ, F.; MOSTAFA, E. M. E. Trust region methods for solving the optimal output feedback design problem. International Journal of Control, v. 76, n. 5, p. 501-519, 2003. Citado na página 28.

LEVINE, W. Optimal Output-feedback Controllers for Linear Systems. Tese (Doutorado), 1969. Citado na página 27.

LEVINE, W.; ATHANS, M. On the determination of the optimal constant output feedback gains for linear multivariable systems. Automatic Control, IEEE Transactions on, v. 15, n. 1, p. 44-48, 1970. Citado 5 vezes nas páginas 27, 34, 58, 62 e 65.

LEWIS, F. L.; VRABIE, D.; SYRMOS, V. L. Optimal control. [S.l.]: John Wiley \& Sons, 2012. Citado na página 49.

MAKILA, P. M.; TOIVONEN, H. T. Computational methods for parametric LQ problems - a survey. Ieee Transactions on Automatic Control, v. 32, n. 8, p. 658-671, 1987. Citado na página 27.

MATLAB. Constrained Nonlinear Optimization Algorithms: User's Guide (R2015a). MathWorks, 2015. Disponível em: <http://www.mathworks.com/help/optim/ug/ constrained-nonlinear-optimization-algorithms.html\#f26622>. Citado na página 63.

MEIROVITCH, L. Principles and techniques of vibrations. [S.1.]: Prentice Hall New Jersey, 1997. v. 1. Citado 3 vezes nas páginas 37, 42 e 45.

MEIROVITCH, L.; BARUH, H. On the inclusion principle for the hierarchical finite-element method. International Journal for Numerical Methods in Engineering, v. 19, n. 2, p. 281-291, 1983. Citado na página 49.

MEIROVITCH, L.; BARUH, H.; OZ, H. A comparison of control techniques for large flexible systems. Journal of Guidance Control and Dynamics, v. 6, n. 4, p. 302-310, 1983. Citado na página 69.

MODAL SHOP, I. T. Miniature electrodynamic shakers models 2004E / 2007E. [S.1.], 2009. Citado na página 70. 
MOERDER, D.; CALISE, A. Convergence of a numerical algorithm for calculating optimal output feedback gains. Automatic Control, IEEE Transactions on, v. 30, n. 9, p. 900-903, 1985. Citado na página 28.

MOON, S. H. Finite element analysis and design of control system with feedback output using piezoelectric sensor/actuator for panel flutter suppression. Finite Elements in Analysis and Design, v. 42, n. 12, p. 1071-1078, 2006. Citado na página 31.

NOCEDAL, J.; WRIGHT, S. J. Numerical Optimization. 2nd. ed. [S.l.]: Springer, 2008. Citado na página 63.

OMEGA. Accelerometers: ACC101, ACC102A, ACC103, ACC301A, ACC310, ACC320 (User's Guide). [S.l.], 2017. 27 p. Citado na página 68.

POLYAK, B. T.; SHCHERBAKOV, P. S. Hard problems in linear control theory: Possible approaches to solution. Automation and Remote Control, v. 66, n. 5, p. 681-718, 2005. Citado na página 29.

PREUMONT, A. Vibration control of active structures: an introduction. [S.l.]: Springer Science \& Business Media, 2011. v. 179. Citado na página 83.

RAUTERT, T.; SACHS, E. W. Computational design of optimal output feedback controllers. Siam Journal on Optimization, v. 7, n. 3, p. 837-852, 1997. Citado na página 28.

REDDY, J. N. An introduction to the finite element method. 3rd. ed. [S.l.]: McGraw-Hill, 2006. Citado na página 48.

SADABADI, M. S.; PEAUCELLE, D. From static output feedback to structured robust static output feedback: A survey. Annual Reviews in Control, v. 42, p. 11-26, 2016. Citado 2 vezes nas páginas 29 e 55.

STRANG, G. Linear Algebra and Its Applications. [S.l.]: Thomson, Brooks/Cole, 2006. Citado na página 45.

STRANG, G.; FIX, G. J. An analysis of the finite element method. [S.l.]: WellesleyCambridge, 2008. Citado na página 49.

SYRMOS, V. L. et al. Static output feedback - A survey. Automatica, v. 33, n. 2, p. 125-137, 1997. Citado na página 29.

TAFLOVE, A.; HAGNESS, S. C. Computational electrodynamics: the finite-difference time-domain method. [S.1.]: Artech house, 2005. Citado na página 41.

TOIVONEN, H. T. A globally convergent algorithm for the optimal constant outputfeedback problem. International Journal of Control, v. 41, n. 6, p. 1589-1599, 1985. Citado na página 28.

TOIVONEN, H. T.; MAKILA, P. M. A descent anderson-moore algorithm for optimal decentralized control. Automatica, v. 21, n. 6, p. 743-744, 1985. Citado na página 28.

ZABINSKY, Z. B. et al. Improving hit-and-run for global optimization. Journal of Global Optimization, v. 3, n. 2, p. 171-192, 1993. Citado na página 31. 
ZHOU, P. X. et al. Optimal construction and control of flexible manipulators: a case study based on LQR output feedback. Mechatronics, v. 11, n. 1, p. 59-77, 2001. Citado na página 30. 